Prepared for the U.S. Department of Energy

under Contract DE-AC05-76RL01830

\title{
Remedy Evaluation Framework for Inorganic, Non-Volatile Contaminants in the Vadose Zone
}

\author{
MJ Truex \\ KC Carroll
}

May 2013

\section{Pacific Northwest}

NATIONAL LABORATORY

Proudly Operated by Battelle Since 1965 


\title{
DISCLAIMER
}

This report was prepared as an account of work sponsored by an agency of the United States Government. Neither the United States Government nor any agency thereof, nor Battelle Memorial Institute, nor any of their employees, makes any warranty, express or implied, or assumes any legal liability or responsibility for the accuracy, completeness, or usefulness of any information, apparatus, product, or process disclosed, or represents that its use would not inf ringe privately owned rights. Reference herein to any specific commercial product, process, or service by trade name, trademark, manufacturer, or otherwise does not necessarily constitute or imply its endorsement, recommendation, or favoring by the United States Government or any agency thereof, or Battelle Memorial Institute. The views and opinions of authors expressed herein do not necessarily state or reflect those of the United States Government or any agency thereof.

\author{
PACIFIC NORTHWEST NATIONAL LABORATORY \\ operated by \\ BATTELLE \\ for the \\ UNITED STATES DEPARTMENT OF ENERGY \\ under Contract DE-AC05-76RL01830
}

Printed in the United States of America
Available to DOE and DOE contractors from the
Office of Scientific and Technical Information
P.O. Box 62, Oak Ridge, TN 37831-0062;
ph: (865) 576-8401
fax: $(865) 576-5728$
email: reports@adonis.osti.gov

Available to the public from the National Technical Information Service

5301 Shawnee Rd., Alexandria, VA 22312

ph: (800) 553-NTIS (6847)

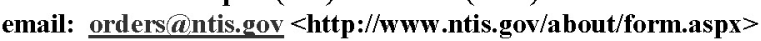

Online ordering: http://www.ntis.gov

This document was printed on recycled paper. 
PNNL-21815

RPT-DVZ-AFRI-004

\title{
Remedy Evaluation Framework for Inorganic, Non-Volatile Contaminants in the Vadose Zone
}

\author{
MJ Truex \\ KC Carroll
}

May 2013

Prepared for

the U.S. Department of Energy

under Contract DE-AC05-76RL01830

Pacific Northwest National Laboratory

Richland, Washington 99352 


\begin{abstract}
Contaminants in the vadose zone may be a long-term source of groundwater contamination and need to be considered in remedy evaluations. In many cases, remediation decisions for the vadose zone will need to be made all or in part based on projected impacts to groundwater. There are significant natural attenuation processes inherent in vadose zone contaminant transport. Attenuation processes include both hydrobiogeochemical processes that serve to retain contaminants within porous media and physical processes that slow the vertical rate of movement for disposed water and waste solutions. In particular, the physical processes controlling fluid flow in the vadose zone are quite different and generally have a more significant attenuation impact on contaminant transport relative to those within the groundwater system. A remedy evaluation framework is presented herein that uses an adaptation of the established EPA Monitored Natural Attenuation evaluation approach integrated into an approach based on a conceptual model focused on identifying and quantifying features and processes that control contaminant flux through the vadose zone. This report presents a framework to support evaluation of contaminant fate and transport in the vadose zone and bridges this analysis to refinement of the site conceptual model, assessment of risk, and support for baseline risk assessment and remedy selection.
\end{abstract}




\section{Acknowledgments}

This document was prepared by the Deep Vadose Zone-Applied Field Research Initiative at Pacific Northwest National Laboratory. Funding for this work was provided by the U.S. Department of Energy Richland Operations Office. The Pacific Northwest National Laboratory is operated by Battelle Memorial Institute for the U.S. Department of Energy under Contract DE-AC05-76RL01830. 


\section{Acronyms and Abbreviations}

$\begin{array}{ll}\text { CCU } & \text { Cold Creek Unit } \\ \mathrm{cm} & \text { centimeter(s) } \\ \text { DOE } & \text { U.S. Department of Energy } \\ \text { DWS } & \text { drinking water standard } \\ \text { EPA } & \text { U.S. Environmental Protection Agency } \\ \text { ITRC } & \text { The Interstate Technology \& Regulatory Council } \\ \text { L } & \text { liter(s) } \\ \text { m } & \text { meter(s) } \\ \text { MNA } & \text { Monitored Natural Attenuation } \\ \text { OSWER } & \text { EPA Office of Solid Waste and Emergency Response } \\ \text { SAC } & \text { System Assessment Capability } \\ \text { U Pond } & \text { Hanford 200 Area 216-U-10 site }\end{array}$




\section{Contents}

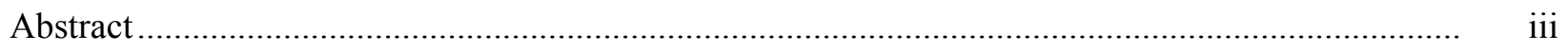

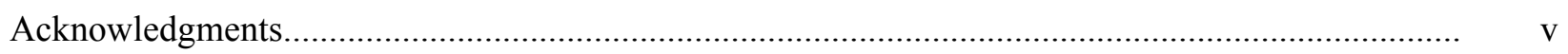

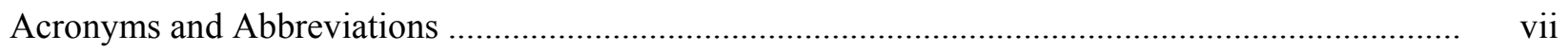

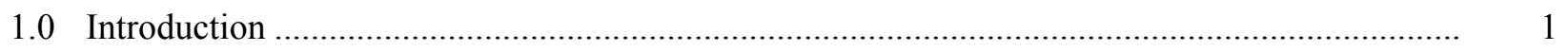

2.0 Vadose Zone Remedy Evaluation Framework .....................................................................

2.1 Vadose Zone Transport Basis of the Remedy Evaluation Framework .............................. 3

2.1.1 Hydraulic Processes Impacting Movement of Added Water .................................... 3

2.1.2 Dispersive Processes Impacting Contaminant Movement ..................................... 7

2.1.3 Waste Fluid Properties Impact on Hydraulic and Biogeochemical Processes ......... 8

2.1.4 Contaminant Solubility, Sorption, and Degradation/Decay Processes..................... 9

2.2 Monitored Natural Attenuation Basis of the Remedy Evaluation Framework .................. 10

2.3 Remedy Evaluation Framework: Evaluation Approach................................................. 18

3.0 Vadose Zone Transport Concepts................................................................................ 20

3.1 Physical Transport Processes and Controlling Features..................................................... 20

3.1.1 Moisture Retention, Capillary Trapping, and Gravity Drainage ............................. 20

3.1.2 Darcy's Law, Hydraulic Conductivity, and Relative Permeability ......................... 22

3.1.3 Unsaturated Infiltration and Recharge ............................................................ 22

3.1.4 Heterogeneous Permeability Impacts on Infiltration........................................... 24

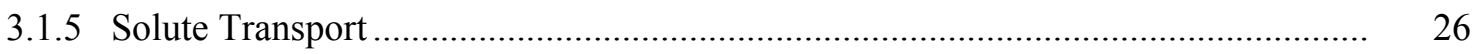

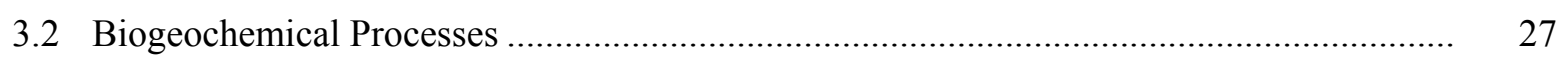

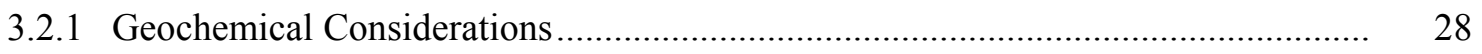

3.2.2 Considerations for Biologically Mediated Processes ........................................... 28

4.0 Potential Applications and Case Study Examples ................................................................ 28

5.0 Characterization and Monitoring Approaches Supporting Remedy Implementation................. 49

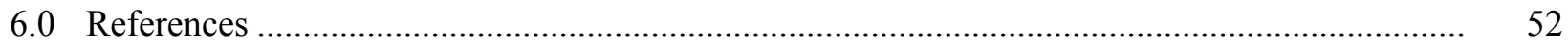




\section{Figures}

1 Conceptual depiction of waste discharge moving through a vadose zone................................. 5

2 Conceptual example relating saturation to relative water flux to groundwater in comparison to the water flux to groundwater expected for recharge-driven conditions............... 6

3 Conceptual depiction of processes related to the impact of localized high vertical permeability zones on vertical transport of added contaminated water in the vadose zone ......... 7

4 Conceptual depiction of pore-scale processes that can contribute to contaminant plume dispersion in the vadose zone

5 Conceptual depiction of generalized water and contaminant fluxes to groundwater resulting from a pulse input of water and contaminants to a vadose zone................................. 10

6 Vadose zone remedy evaluation and implementation flow chart

7 Attenuation mechanisms for inorganic contaminants in groundwater and factors that can impact attenuation

8 Attenuation mechanisms for inorganic contaminants in the vadose zone and factors that can impact attenuation .

9 Illustrative soil characteristic curves for a fine soil and a coarse soil

10 Illustrative plot of water saturation versus depth below land surface for both transient and steady-state infiltration cases

11 Differences in water saturation between 1985 and 1944

12 Differences in water saturation between 2007 and 1944

13 Site map indicating waste site and well locations for both BX and BY areas....

14 Depiction of the estimated uranium inventory in the vadose zone at the BX-102 site.

15 Lithology, stratigraphy, geophysics, and water content versus depth for well 299-E33-45

16 Lithology, stratigraphy, geophysics, and pore-water concentrations versus depth for well 299-E33-45

17 Water and acid extractable U concentrations with depth at BX-102 and at well 299-E33-45 .....

18 Matric potential by filter paper technique on core and grab samples with depth along well 299-E33-45 .

19 Matric potential by filter paper technique on core samples with depth along well 299-E33-338

20. Lithology, stratigraphy, and moisture and Tc-99 profiles at the 299-W23-19 borehole at the edge of the SX tank area

21 Moisture and Tc-99 profiles at the slant borehole beneath the SX-108 tank.

22 Laboratory-sample-derived Tc-99 and moisture profiles for borehole 5923 located between four cribs and borehole 4191 located adjacent to the B-26 trench at the BC Cribs and Trenches site.

23 Estimated lateral distribution of ionic strength in the vadose zone at the BC Cribs and Trenches site

24 Simulated water flux across the water table and predicted Tc-99 concentrations over time in the groundwater beneath the trenches portion of the BC Cribs and Trenches site 
25 Depiction of the estimated technetium inventory in the vadose zone at BY Cribs using data from Serne et al. (2010)

26 Lithology, total gamma, and water content versus depth for well 299-E33-342 in the BY area

27 Electrical resistivity survey depicting lateral extent of low resistivity expected to correspond with the vadose zone contaminant distribution

28 Electrical resistivity survey depicting a vertical slice of the subsurface where the lowresistivity region is expected to correspond with the vadose zone contaminant distribution

29 Tc-99 concentration profiles at the BY-49 Crib and well 299-E33-341...

30 Groundwater concentrations over time of nitrate and Tc-99 at well 299-E33-7 in the BY area

31 Generic description of the vadose zone as a transport path from the surface to groundwater for non-volatile contaminants

\section{Tables}

1 Summary of MNA assessment criteria typically used for groundwater contamination sites and proposed for the vadose zone

2 Categorization of vadose zone sites based on potential impact to groundwater..... 


\subsection{Introduction}

This document provides a remedy evaluation framework for inorganic, non-volatile contaminants in the vadose zone. The framework is focused on sites where protection of groundwater is the remediation objective for vadose zone contamination, specifically for sites where waste disposal and aqueous discharges have distributed contamination within the vadose zone (e.g., buried solid waste is not specifically discussed). Application of the framework is intended to work in concert with fate and transport modeling used to establish groundwater protection remediation goals, to fit within the Remedial Investigation/Feasibility Study process (or a Corrective Measures Study), support the baseline risk assessment and associated evaluation of the no-action alternative, and, as needed, evaluation of remediation alternatives. The premise for this framework is that a structured approach to evaluating contamination in the vadose zone is useful because, in many cases, remediation decisions for the vadose zone will need to be made all or in part based on projected impacts to groundwater. Another guiding premise is recognition that significant natural attenuation of waste fluids added to the vadose zone can occur and decrease the impact to groundwater because contaminant concentrations and the temporal profile of contaminant flux to groundwater are decreased by multiple processes in the vadose zone. Thus, transport through the vadose zone is impacted by natural attenuation processes, and remediation in the vadose zone to protect groundwater is functionally a combination of natural attenuation and other remediation techniques, as needed, to further mitigate contaminant flux to groundwater. Because of the potentially significant contribution of natural attenuation for transport of contaminants in the vadose zone and the emphasis of remediation on mitigating contaminant flux to groundwater, the remedy evaluation framework presented herein is structured to use an adaptation of the established U.S. Environmental Protection Agency (EPA) Monitored Natural Attenuation (MNA) evaluation approach and a conceptual model based-approach focused on identifying and quantifying features and processes that control contaminant flux through the vadose zone.

MNA is a remedy defined by the EPA Office of Solid Waste and Emergency Response (OSWER) in "Use of Monitored Natural Attenuation at Superfund, RCRA Corrective Action, and Underground Storage Tank Sites" (OSWER Directive 9200.4-17P; EPA 1999). The OSWER MNA Directive applies to soils and groundwater and defines natural attenuation processes included in an MNA approach as follows:

The "natural attenuation processes" that are at work in such a remediation approach [MNA] include a variety of physical, chemical, or biological processes that, under favorable conditions, act without human intervention to reduce the mass, toxicity, mobility, volume, or concentration of contaminants in soil or groundwater. These in situ processes include biodegradation; dispersion; dilution; sorption; volatilization; radioactive decay; and chemical or biological stabilization, transformation, or destruction of contaminants.

For selection of MNA as a remedy, the OSWER MNA Directive describes three progressive elements that need to be satisfied. The level of detail and number of elements needed at a given site depend on the site conditions and risk as determined by the EPA or the overseeing regulatory authority. Thus, at some sites, only element 1 is required. At other sites, all three elements may be required. 
The elements are as follows:

\begin{abstract}
(1) Historical groundwater and/or soil chemistry data that demonstrate a clear and meaningful trend of decreasing contaminant mass and/or concentration over time at appropriate monitoring or sampling points. (In the case of a groundwater plume, decreasing concentrations should not be solely the result of plume migration. In the case of inorganic contaminants, the primary attenuating mechanism should also be understood.)
\end{abstract}

(2) Hydrogeologic and geochemical data that can be used to demonstrate indirectly the type(s) of natural attenuation processes active at the site, and the rate at which such processes will reduce contaminant concentrations to required levels. For example, characterization data may be used to quantify the rates of contaminant sorption, dilution, or volatilization, or to demonstrate and quantify the rates of biological degradation processes occurring at the site.

(3) Data from field or microcosm studies (conducted in or with actual contaminated site media) which directly demonstrate the occurrence of a particular natural attenuation process at the site and its ability to degrade the contaminants of concern (typically used to demonstrate biological degradation processes only).

Although the OSWER MNA Directive provides the overall structure for application of MNA at a site, the EPA has published technical protocols that provide more specific details for the process of evaluating and implementing MNA. These protocols are the functional processes that sites must follow for MNA remedies. A technical protocol for MNA assessment has recently been published for inorganic and radionuclide contaminants in groundwater (EPA 2007a, 2007b, 2010). This protocol provides a useful structure from which to develop a suitable conceptual model of the site, identify natural attenuation processes, and estimate contaminant fate and transport relative to meeting remediation goals. These products from an MNA evaluation are also important for use in assessing the need for and determining the best approach to augment natural attenuation to meet remediation goals. Specific guidance for vadose zone inorganic and radionuclide contaminants has not been published by the EPA. However, an effective conceptual model and structured approach to estimate contaminant fate and transport, including consideration of natural attenuation processes, are needed in the vadose zone to evaluate remedies that can be protective of groundwater.

This report discusses the extension of the existing groundwater-based MNA protocol (EPA 2007a) to support the evaluation of contaminant transport through the vadose zone and the associated natural attenuation of contaminants within the vadose zone. Evaluation of transport is structured to facilitate refinement of the site conceptual model and assessment of risk, and to provide support for baseline risk assessment and remedy selection. This approach supports consideration of active remedies to augment natural attenuation within the context of the recognized "enhanced attenuation" approach (ITRC 2010; Truex et al. 2011a). The fluid flow and transport processes in the vadose zone are very different from those within the groundwater system, and many of these important differences are discussed in the subsequent sections of this report. However, with modification, the general framework for assessment of MNA that has been proposed for metals and radionuclides in groundwater can be transferrable to evaluation of vadose zone contaminant transport and risk to groundwater. Developing the vadose zone evaluation framework with the same structure and approach as the groundwater MNA framework was 
done to ensure consistency in application and documentation for both groundwater and vadose zone evaluations and to support regulatory acceptance and application.

The remedy evaluation framework is described in Section 2 along with presentation of concepts based on vadose zone transport characteristics (Section 2.1) and approaches based on lines-of-evidence, conceptual models, and other key elements of MNA (Section 2.2) that were used in development of the framework. Section 3 provides additional background on vadose zone transport concepts that provide technical support for this approach. Discussion of case study applications of the approach is provided in Section 4. Potentially appropriate characterization and monitoring compatible with this approach are discussed in Section 5.

\subsection{Vadose Zone Remedy Evaluation Framework}

A remedy evaluation framework for inorganic, non-volatile contaminants in the vadose zone was developed that is linked to vadose zone transport characteristics (Section 2.1). The framework uses a lines-of-evidence, conceptual-model-based approach and other key concepts from MNA (Section 2.2) and is based on evaluating remedies in terms of mitigating contaminant flux to groundwater (Section 2.3).

\subsection{Vadose Zone Transport Basis of the Remedy Evaluation Framework}

In the context of protecting groundwater, transport rates of disposed or otherwise discharged contaminants (typically at surface or near surface locations) through the vadose zone control the contaminant flux to groundwater which, along with groundwater mixing and attenuation processes, impact the contaminant concentrations within any resulting groundwater plume. Several key processes in the vadose zone affect contaminant transport. Hydraulic processes in the vadose zone can significantly alter the transport rate of contaminants along a flow path from a discharge location to the groundwater because of the characteristics of water movement in unsaturated environments (Section 2.1.1). With long residence times and unsaturated conditions in the vadose zone, dispersive processes may impact contaminant movement through the vadose zone (Section 2.1.2). In some cases, disposed waste may have physical properties that impact how contaminants move through the vadose zone (Section 2.1.3). Similar to considerations in groundwater, contaminant solubility, sorption, and degradation/decay processes are important to contaminant fate and transport (Section 2.1.4). The following sections examine these important processes in the vadose zone in the context of evaluating how the processes impact contaminant flux to groundwater and providing a contextual basis for quantifying this flux.

\subsubsection{Hydraulic Processes Impacting Movement of Added Water}

Unsaturated flow processes and potential preferential flow phenomena can impact movement of added water through the vadose zone and to the groundwater. 


\subsubsection{Unsaturated Flow Processes}

Unless water discharged as part of waste disposal or associated with other discharges such as leakage from engineered systems is large compared to the thickness of the vadose zone, water flow through the vadose zone will be slowed via unsaturated flow processes. Further, in most cases, water discharged in association with waste disposal will occur over a finite timeframe and thus represent a "pulse" input to the vadose zone. Figure 1 depicts several scenarios of contaminated water discharge to the vadose zone and progression of added water movement over time that results in a contaminant flux to groundwater. Figure 1 focuses on the movement of the added water to highlight key hydraulic considerations related to contaminant movement through the vadose zone. The depicted flux to groundwater would need to be further evaluated based on contaminant solubility, sorption, and degradation/decay processes (see Section 2.1.4). Figure 1 does not include the potential impact of preferential flow features; these features are discussed later in this section.

Key features of hydraulic processes in the vadose zone related to contaminant flux to groundwater can be inferred from the scenarios depicted in Figure 1.

- Lateral spreading of water in unsaturated conditions reduces the amount of a contaminated water pulse added to the vadose zone that can move vertically at a rate faster than the rate of water movement under recharge-driven (precipitation rather than waste-driven) conditions. Lateral spreading decreases the moisture content and associated water pressure and thereby is a hydraulic process that dissipates the added water hydraulic driving force trending conditions back toward those associated with recharge-driven water movement rates. In the unsaturated zone, hysteresis in wetting/ drying curves can also hasten dissipation of water pressure from added water back toward pressure gradients associated with recharge-driven conditions and thereby also may reduce the amount of a contaminated water pulse that can move vertically at a rate faster than the rate under recharge-driven conditions. Thus, quantifying lateral spreading and hysteresis conditions are key elements for understanding the movement of a contaminated water pulse through the vadose zone.

- As shown in Figure 1, there are characteristic curves of the flux to groundwater based on the magnitude of the added water pulse in relation to the vadose zone properties and the hydraulic properties of the vadose zone. In the worst case for an added water pulse, there is a pulse of increased flux to the groundwater over a limited time related to the waste disposal. All cases relax to a water flux that has a magnitude controlled by the recharge rate. Thus, quantifying the recharge rate is important as part of estimating long-term flux to groundwater. Efforts to estimate the expected characteristic shape and key elements that define the profile of the water flux to groundwater are important to evaluating overall impact to the groundwater.

- Figure 1 depicts a special case where perched water features create flux conditions related to the perching zone properties and distance from the perched zone to groundwater. Under most conditions, if the added water becomes perched, there was already perched water or high-saturation conditions in that zone under recharge-driven conditions. Thus, the perched zone may not need to be completely dewatered to return to a flux on the order of the recharge rate.

- Vadose zone hydraulic processes act to naturally attenuate water flow from a discharge location to the groundwater. Thus, by evaluating the vadose zone hydrologic conditions in the context of information about the waste discharge, the character of the flux to groundwater can be estimated as part of accounting for natural attenuation processes in the vadose zone. 


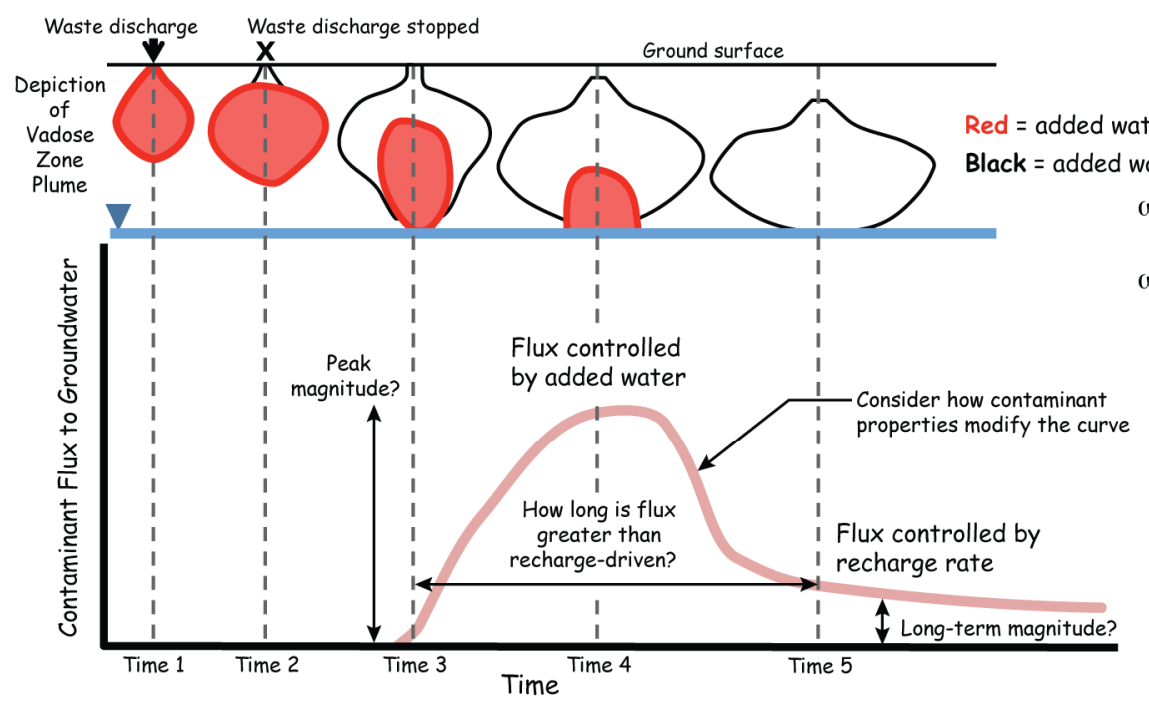

Red $=$ added water moving faster than recharge $\left(\omega \gg \omega_{r}\right)$
$\omega=$ the moisture content for recharge- driven water flux conditions.

$\omega_{r}=$ the nominal moisture content for recharge-driven water flux conditions.

Waste discharge moving through the vadose zone with a large amount of added water compared to vadose zone thickness and/or lateral spreading.

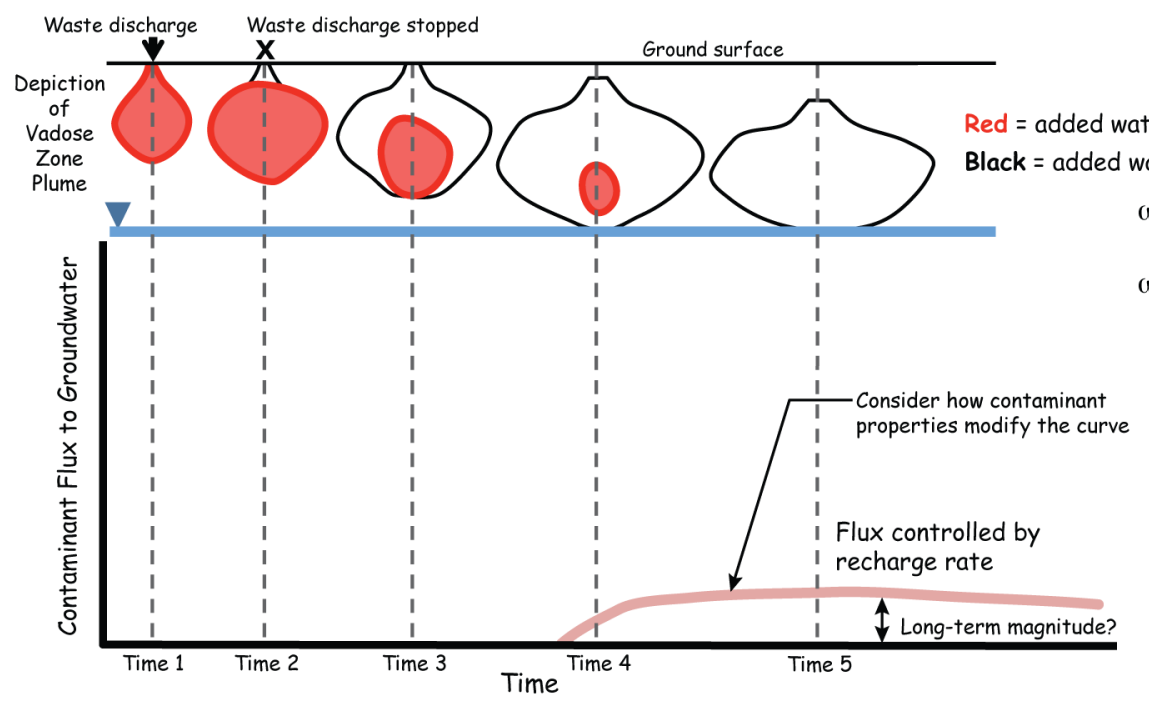 driven water flux conditions.
$\omega_{r}=$ the nominal moisture content for recharge-driven water flux conditions.

$\omega=$ the moisture content for recharge-

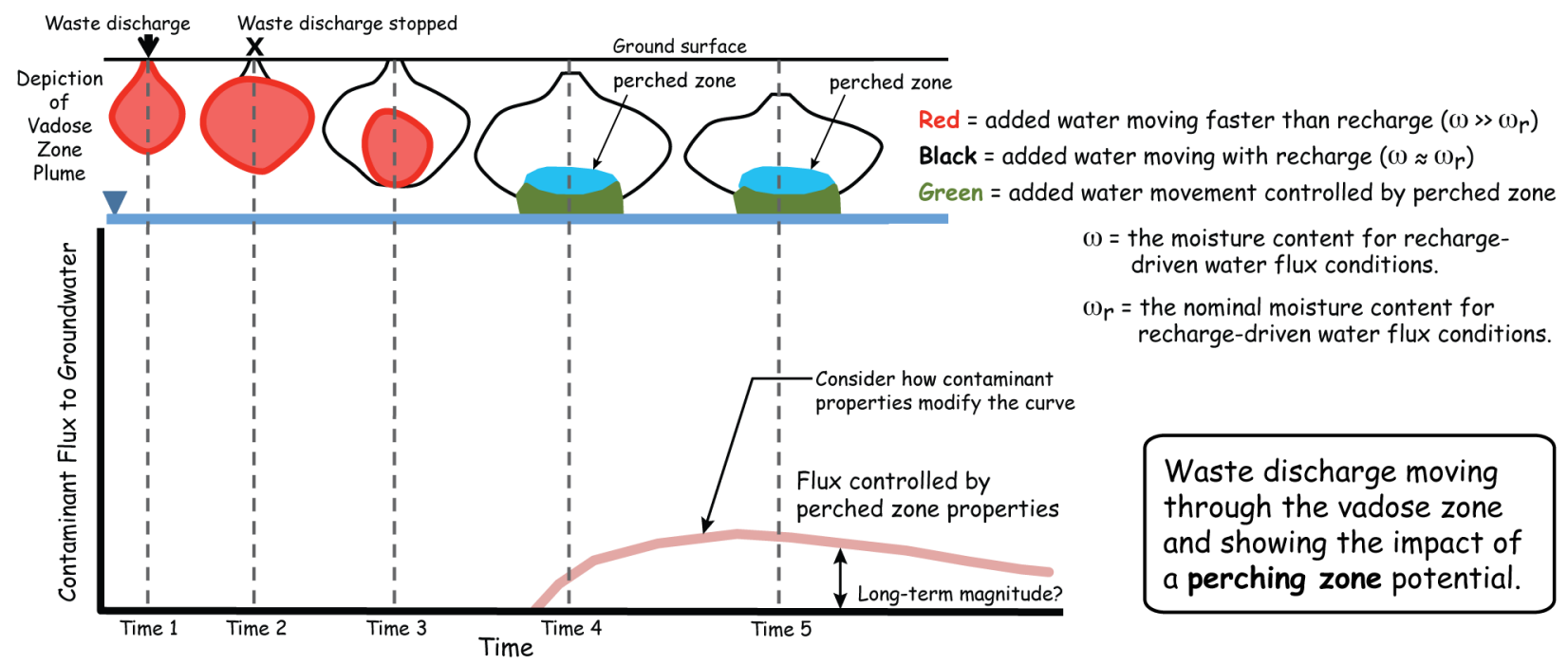
Waste discharge moving through the vadose zone with a small amount of added water compared to vadose zone thickness and/or lateral spreading.

Figure 1. Conceptual depiction of waste discharge moving through a vadose zone (A - top, B - middle, $\mathrm{C}$ - bottom). 
To estimate the unsaturated flow conditions depicted in Figure 1 for a given site, it may be useful to assess bounding values for the vertical water flow and associated water flux to groundwater based on the properties of unsaturated flow. These bounding assessments can consider relative draining and rechargedriven conditions in the vadose zone as depicted in Figure 2 along with the vertical profile of conditions at the site (i.e., a 1-dimensional assessment of a site using the type of information for sediment properties and moisture profiles that can be obtained from a borehole). For sites where a water pulse was added, post-discharge conditions will tend to move along the curve toward the recharge-driven conditions over time. With appropriate sediment properties and an assessment of the recharge-driven flux conditions for the site, flux to groundwater can be bound to values between the recharge-driven flux and the flux that corresponds to current or expected maximum saturation conditions in the vadose zone. These values then relate to the characteristic shape of the flux curves shown on Figure 1A and Figure 1B. A separate assessment would be needed to evaluate the movement of water out of a perched zone. Site data can potentially be used to determine the current position on the saturation/flux curve and thereby assess the expected changes in the future as the site relaxes back toward recharge-driven conditions.

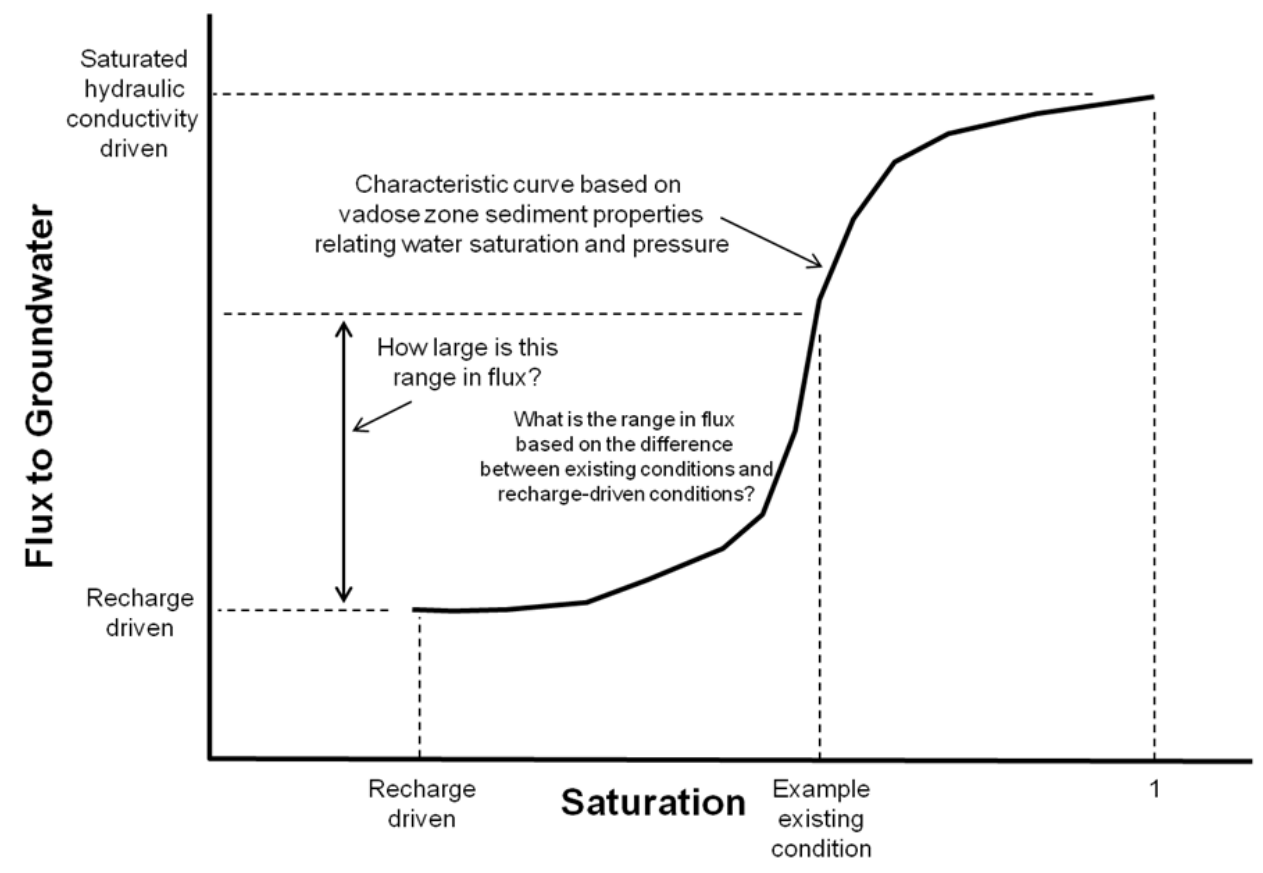

Figure 2. Conceptual example relating saturation to relative water flux to groundwater in comparison to the water flux to groundwater expected for recharge-driven conditions.

In Figure 1A, it is also of importance to estimate the timeframe over which the flux to groundwater was or is expected to be greater than the expected long-term recharge-driven flux. Three elements of this type of assessment include 1) the current groundwater contaminant plume data (if present), 2) the water saturation profile along the centerline of the vadose zone migration path and related position along the curve shown in Figure 2 with respect to reaching recharge-driven conditions, and 3) numerical modeling assessments to estimate potential flux profiles based on estimates of waste discharge and vadose zone properties. 


\subsubsection{Preferential Flow Phenomena}

Some hydrologic features in the vadose zone have higher vertical permeability than the surrounding porous media and locally increase vertical transport of added water. Figure 3 depicts a high vertical permeability feature and processes that relate to its impact on movement of added water at different times during and after waste discharge. As noted in Figure 3, while vertical movement is enhanced during high saturation discharge conditions, the impact of the preferential flow path diminishes as saturation declines, for instance in the period after waste discharge has been terminated. The impact of vertical features is related to the properties of the feature in relation to the surrounding porous media matrix (e.g., whether the matrix of the features is a coarser or finer medium or is a fracture) and the moisture conditions. For instance, relative water flux through clastic dike vertical features compared to the surrounding porous media is a function of the moisture conditions (Murray et al. 2003).

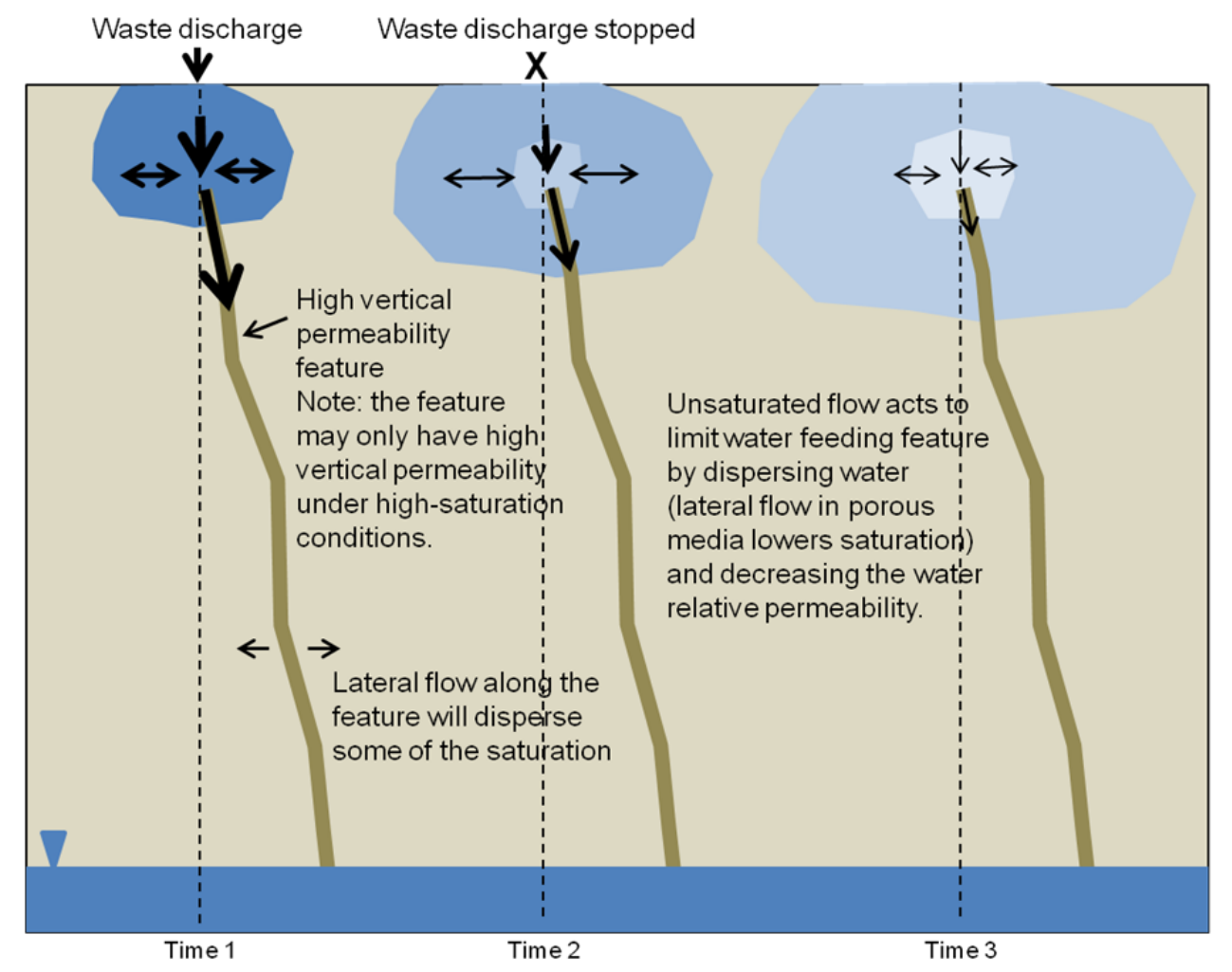

Figure 3. Conceptual depiction of processes related to the impact of localized high vertical permeability zones on vertical transport of added contaminated water in the vadose zone.

\subsubsection{Dispersive Processes Impacting Contaminant Movement}

Due to tortuosity, capillary forces, and intra-particle water, significant dispersion can occur during movement of contaminants through the vadose zone with some of the relevant processes depicted in Figure 4. Contaminated water may enter pores on wetting and then not readily drain due to hysteresis for draining compared to wetting. Diffusion of contaminants into pores that are functionally immobile zones due to tortuosity or presence as intra-particle pores disperses contaminants and lowers concentrations, leading to longer-term diffusion control of contaminants moving out of these functionally immobile zones 
and overall dispersion of the contaminant plume. Mixing of added water and existing pore water can also dilute contaminant concentrations, causing overall dispersion of the contaminant plume.

In addition to these pore-scale processes, larger-scale dispersion can occur due to the hydraulic processes discussed in Section 2.1.1, especially as induced by variations in hydraulic properties along the flow path through the vadose zone.

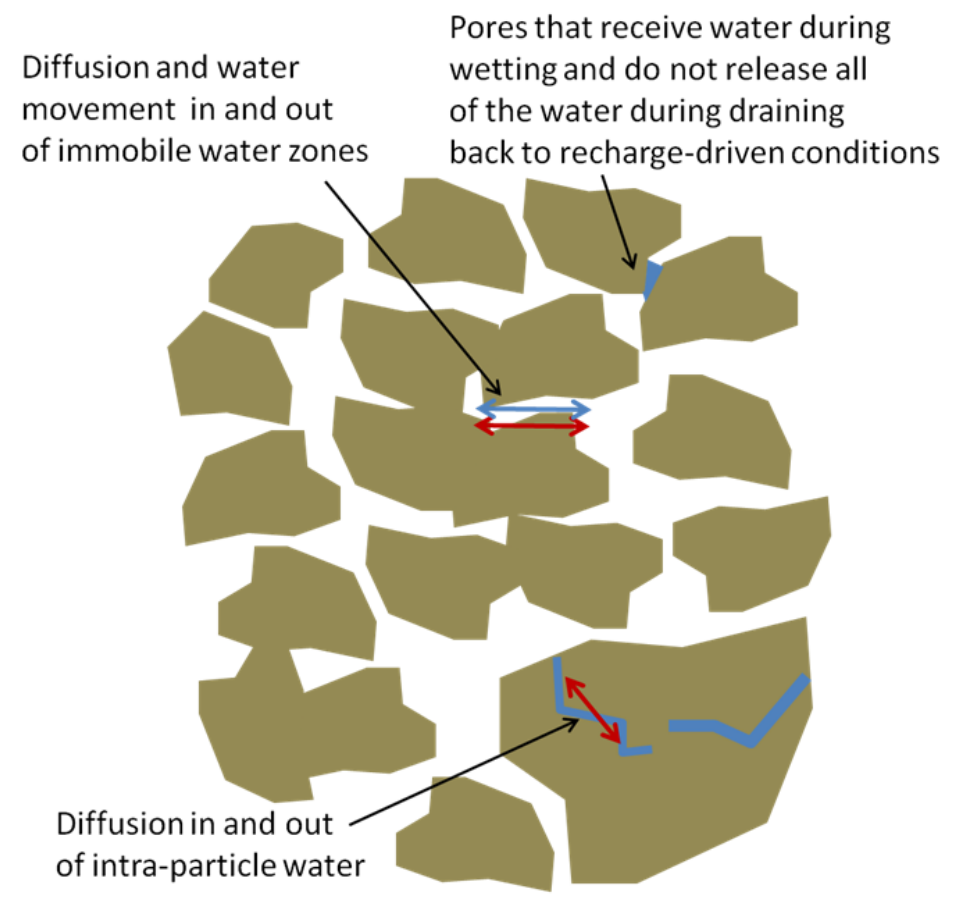

Figure 4. Conceptual depiction of pore-scale processes that can contribute to contaminant plume dispersion in the vadose zone.

\subsubsection{Waste Fluid Properties Impact on Hydraulic and Biogeochemical Processes}

Typically, wastes are disposed/discharged directly into the vadose zone (e.g., at the surface), and the waste fluid physical and chemical properties may influence hydraulic and biogeochemical processes for a portion of the vadose zone, depending on the nature and magnitude of these properties in comparison to the conditions within the vadose zone prior to disposal. Key waste fluid properties are listed below along with potential impacts on contaminant transport through the vadose zone. These factors are not included directly in this document but can be qualitatively evaluated based on the information below to determine the need to conduct more detailed site-specific analysis for sites where the impact of waste fluid properties may be significant.

- Fluid Density - Waste solutions with higher density than that of the pore water will tend to have increased vertical versus lateral movement compared to pore water.

- Viscosity - Waste solutions with higher viscosity than that of the pore water will tend to move slower with more fluid retention in the vadose zone and have increased vertical versus lateral movement compared to pore water. 
- Solute Concentration - Waste solutions with higher solute concentrations may promote more diffusion of contaminants into pore water that is contacted during transport. In addition, high solute concentrations may impact sorption and solubility reactions.

- Non-Aqueous Phase Liquid Waste - Presence of a non-aqueous phase liquid (e.g., as a co-contaminant with inorganic, non-volatile contaminants) can complicate water flow processes and impact sorption and solubility reactions.

\subsubsection{Contaminant Solubility, Sorption, and Degradation/Decay Processes}

Contaminant transport is impacted by solubility, sorption, and degradation/decay processes that are a function of the contaminant properties and the biogeochemistry in the vadose zone. These processes are essentially the same as those presented and described in detail in the EPA technical protocol for MNA of inorganic contaminants in groundwater (EPA 2007a, 2007b, 2010; ITRC 2010) and described with respect to conceptual site models by Truex et al. (2011a). Potential considerations unique to the vadose zone include the following items:

- presence of a gas phase that may be important for some biogeochemical processes related to its interaction and impact on pore-water chemistry

- potential for more "extreme" chemistry or physical processes due to waste fluid properties as discussed in Section 2.1.3

- potential for long residence times of contaminated pore water that may be more likely to favor equilibrium chemistry conditions and enable slower kinetic reactions to proceed more fully than in a faster-flowing groundwater setting.

These processes need to be evaluated and considered in combination with the factors controlling water flux to the groundwater to estimate the contaminant flux to groundwater. Figure 5 shows a conceptual depiction of the contaminant flux for different types of contaminant behavior (conservative non-sorbing, sorbing, and solubility [kinetic] controlled) compared to the water flux as depicted in Figure 1A (Section 2.1.1). Each individual attenuation process (Figure 5) decreases contaminant concentrations or migration rates such that, over the long term, the combined impact is a cumulative contaminant reduction response that may be greater than the impact of any individual process.

Solubility and degradation/decay process should be considered in terms of reducing the portion of the total discharged contaminant mass that will reach the groundwater. Degradation/decay processes directly reduce contaminant mass unless products of degradation or decay are also contaminants. Solubility reactions that cause contaminant precipitates can also functionally reduce the mass that will reach the groundwater if the precipitate solubility is low and rates of contaminant release over time back into pore water are small enough that concentrations remain below a level that would cause a flux to groundwater that exceeds groundwater protection goals. Solubility reactions, in particular, need to be evaluated 1) in the context of the discharged waste chemistry and initial interactions with the sediments as the waste was released to define precipitates that may form rapidly due to the initial waste chemistry and 2) in the context of pore-water chemistry expected farther from the discharge location that is likely trending toward the pre-disposal pore-water chemistry. 
Sorption processes do not reduce the contaminant mass that will reach the groundwater but delay transport and reduce contaminant concentrations in the vadose zone plume. Sorption is a function of contaminant properties, sediment properties, and pore-water chemistry. The discharged waste chemistry may impact pore-water chemistry (e.g., pH, solute concentrations) and sediment properties (e.g., surface phases due to precipitation/dissolution processes), especially near the waste discharge location. Thus, evaluation of contaminant transport should consider waste chemistry impacts on sorption.

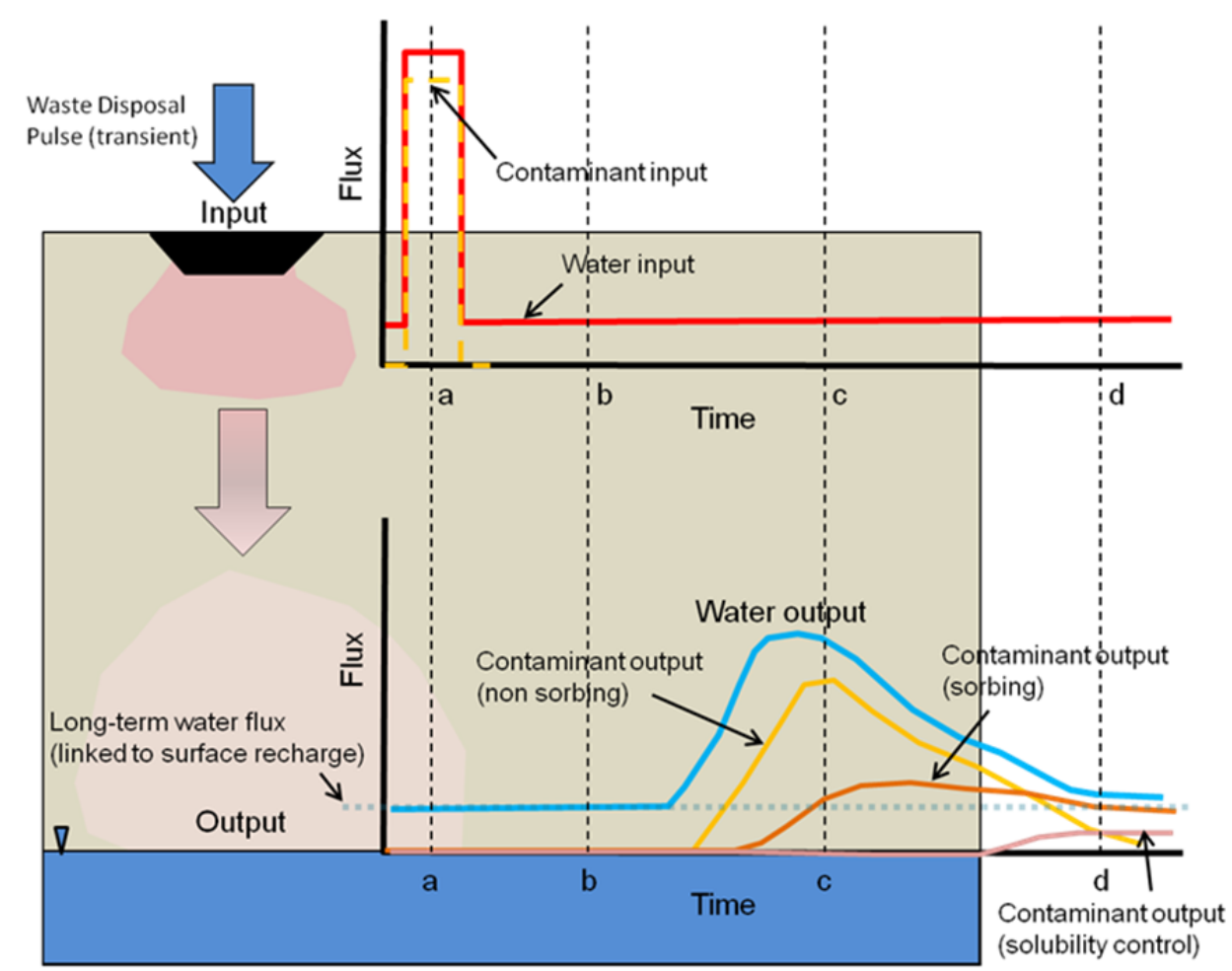

Figure 5. Conceptual depiction of generalized water and contaminant fluxes to groundwater resulting from a pulse input of water and contaminants to a vadose zone.

\subsection{Monitored Natural Attenuation Basis of the Remedy Evaluation Framework}

To support baseline risk assessment, evaluating the need for a remedy, and facilitate remedy decisions, an approach is needed to evaluate natural attenuation processes in the context of contaminant transport and remediation goals. For groundwater contamination, the EPA technical protocol for MNA of inorganic contaminants in groundwater ([EPA 2007a, 2007b, 2010], hereafter referred to as "EPA Protocol") uses three progressive evaluation tiers and a final implementation tier to assess MNA as a remedy for inorganic and radionuclide contamination (Table 1). This tiered assessment approach developed for groundwater plumes can be re-evaluated and used to develop a specific technical basis and the supporting guidance information for the vadose zone. 
Table 1. Summary of MNA assessment criteria typically used for groundwater contamination sites and proposed for the vadose zone.

\begin{tabular}{cll}
\hline Tier & \multicolumn{1}{c}{ Objective } & \multicolumn{1}{c}{ Assessment } \\
\hline I & $\begin{array}{l}\text { Demonstrate active contaminant } \\
\text { removal from groundwater } \\
\text { Determine mechanism and rate of } \\
\text { attenuation }\end{array}$ & Is the plume expanding, static, or contracting? \\
II & $\begin{array}{l}\text { Is the MNA rate sufficient for attaining cleanup in } \\
\text { reasonable time frame? }\end{array}$ \\
III & $\begin{array}{l}\text { Determine system capacity and } \\
\text { stability of attenuation }\end{array}$ & $\begin{array}{l}\text { Is the MNA capacity sufficient and sustainable to } \\
\text { attenuate contaminant mass to below regulatory } \\
\text { objectives? }\end{array}$ \\
IV $\quad \begin{array}{l}\text { Design performance monitoring } \\
\text { program and identify alternative } \\
\text { remedy }\end{array}$ & $\begin{array}{l}\text { Can monitoring be implemented to verify performance } \\
\text { and identify condition changes that may lead to } \\
\text { failure? }\end{array}$ \\
\hline
\end{tabular}

The type of information collected in an MNA-style evaluation is relevant to conducting analyses of contaminant transport through the vadose zone, baseline risk assessment, and consideration of the no-action alternative. In addition, as with groundwater contamination, MNA may be sufficient as the sole vadose zone remedy or can be quantified for inclusion as part of a remedy along with other measures. Along with providing information to refine the site conceptual model, support baseline risk assessment, and evaluating whether MNA is sufficient as a remedy, an MNA-style approach is also a key initial step in remedy evaluation to define the need for and target the scale of other remedial measures. Similar to groundwater assessments, examining contaminant fate and transport in the vadose zone requires evaluating data and making projections of future contaminant behavior to support a decision about whether to implement MNA (the first three tiers of the EPA Protocol) and providing a basis for monitoring design (the final tier in the EPA Protocol).

By using a tiered approach as described in the EPA Protocol for groundwater, an MNA-style approach to evaluation of contaminant transport through the vadose zone can provide similar information to support remedy decisions. However, because contaminant transport in the vadose zone is significantly different from transport in the groundwater, the specific elements under the tiered approach need to be modified from the groundwater approach. The following sections outline the four-tier EPA Protocol for MNA in the groundwater and describe a parallel approach for applying an evaluation of contaminant transport in the vadose zone to support remedy decisions related to controlling contaminant mass flux/discharge into the groundwater. Conducting this structured evaluation refines the conceptual model of the waste site in terms of contaminant fate and transport and provides a framework for overall remedy evaluation considering both the vadose zone and groundwater systems (Figure 6).

The EPA Protocol considers natural attenuation for contaminant plumes in groundwater, and contaminants in the vadose zone would be considered as part of the source to groundwater. However, contaminants in the vadose zone typically originate from a surface or near-surface waste release (e.g., disposal or leak of contaminated material) and then must move through the vadose zone to become a source to the groundwater. Thus, the fate and transport of contaminants in the vadose zone affect the character of this source to groundwater plumes. As in the groundwater, fate and transport of contaminants in the vadose zone can be attenuated due to natural processes that limit contaminant movement. Natural attenuation in the vadose zone is a mechanism of source control relative to the groundwater and could be considered as sufficient to mitigate risk (e.g., a no-action alternative), or MNA may be all or part of a remedy addressing the released waste material. 


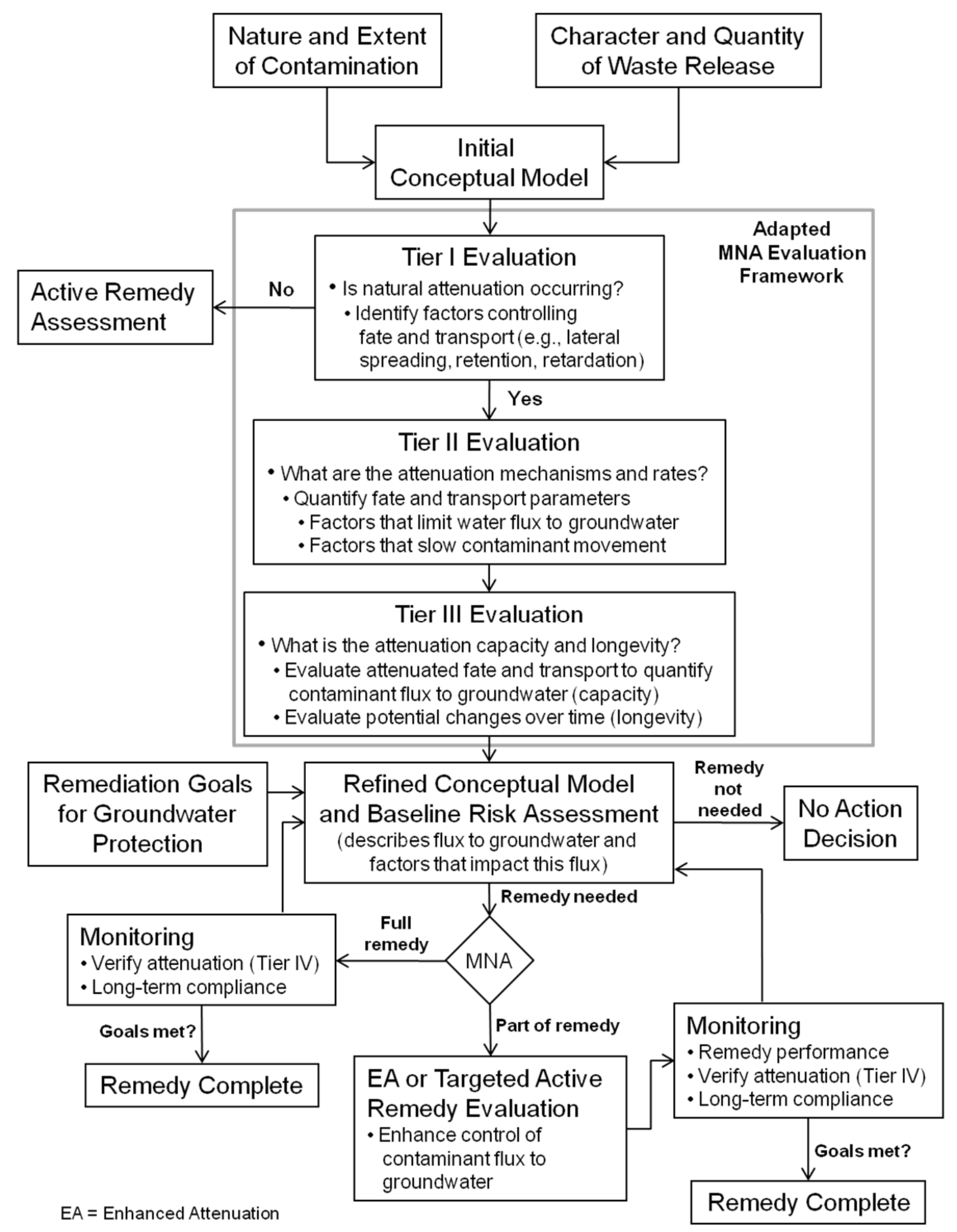

Figure 6. Vadose zone remedy evaluation and implementation flow chart. 
In quantifying the magnitude of a vadose zone source with respect to its impact on groundwater, vadose zone pore-water contaminant concentrations are not directly important; instead, the overall mass discharge of contaminants to the groundwater over time (or the mass flux through a defined crosssectional area) is important. The contaminant mass discharge from the vadose zone in combination with the flow and transport characteristics of the aquifer determines the concentrations within the resultant groundwater contaminant plume. Thus, the role of natural attenuation in the vadose zone is in limiting the contaminant mass flux/discharge to the groundwater.

The vadose zone approach is based on an overall conceptual model framework of contaminant movement in the vadose zone. In this conceptual model, waste material released to the vadose zone is a perturbation of the pre-existing conditions and results in an increase of contaminants and/or moisture in the vadose zone. Due to the nature of moisture movement in the vadose zone, the post-contamination moisture conditions will tend to return toward the pre-existing moisture conditions. The pre-existing conditions are typically governed by the recharge rate, a function of precipitation, transpiration, evaporation, and unsaturated flow processes near the ground surface, resulting in an amount of water added to the vadose zone that continues to move vertically to the groundwater. Under long-term equilibrium conditions (and under conditions where vapor-phase moisture transport is minimal), the rate of water movement is effectively constant throughout the vadose zone and equal to the average recharge rate. When a pulse of water is added (e.g., through waste release), lateral and vertical unsaturated flow affect the movement of discharged water through the vadose zone and into the groundwater and dissipate the vertical movement of water as a function of travel distance and type of porous media contrasts in the vadose zone. Movement of inorganic (non-volatile) dissolved contaminants may be slower than the water movement due to hydrobiogeochemical phenomena such as sorption, solubility, degradation/decay, and dispersive processes. The contaminant discharge to groundwater over time is controlled by the combination of the moisture flux and the contaminant hydrobiogeochemical phenomena. When inorganic contaminants are comingled with non-aqueous phase liquid waste discharges, additional transport phenomena must be considered.

\section{Tier I: Demonstrate Natural Attenuation is Occurring}

The EPA Protocol Tier I evaluation for groundwater plumes uses evidence that natural attenuation is occurring to identify sites for which MNA may be appropriate. Two basic types of information can be used for this assessment in groundwater. Demonstrating that the plume is not expanding, posing a risk to receptors, is a primary approach whereby groundwater monitoring data can be used to define the state of the plume in terms of its behavior over time and its expected behavior in the future. Another approach is to provide evidence that there is active removal of contaminants from groundwater (e.g., sorption or solubility reactions) that are expected to diminish contaminant concentrations over time.

There is no direct exposure risk for a contaminant "plume" within the vadose zone (i.e., pore-water contamination); thus, movement or expansion of the vadose zone plume is a concern only with respect to impacting underlying groundwater. Natural attenuation mechanisms can limit contaminant movement through the vadose zone, and this attenuation in the vadose zone is a mechanism for reducing source flux to the groundwater. In this case, the Tier I assessment for the vadose zone is "Demonstrate that the rate of contaminant movement toward the groundwater is decreased by factors that limit vertical movement of disposed water and/or slow contaminant movement relative to water movement." For many sites, Tier 1 will assessment will show that attenuation is occurring. However, sites with small vadose zone thickness 
compared to discharge conditions or with high recharge may not be able to justify that natural attenuation is functionally limiting contaminant movement in the vadose zone.

\section{Tier I Approaches}

- direct evidence or a transport analysis that demonstrates contaminant movement in the vadose zone has been slowed relative to the rate of release

- data from sediment core samples showing partitioning of contaminants from the pore water to sediment-associated phases has occurred (e.g., comparison of extracted pore-water contaminant mass to contaminant mass remaining with the sediment)

- groundwater monitoring data demonstrating a declining rate of contaminant migration through the vadose zone based on declining contaminant concentrations.

\section{Tier II: Understand and Quantify Natural Attenuation Mechanisms}

The EPA Protocol Tier II evaluation for groundwater plumes requires determining the mechanisms and rates of attenuation. Efforts for groundwater plume assessment would typically focus on data and analysis of geochemical or biogeochemical processes of contaminant attenuation. In particular, contaminant sorption and solubility processes that contribute to attenuation of contaminant transport need to be quantified in the context of the groundwater flow system and geochemical conditions. Figure 7 summarizes the categories of groundwater attenuation mechanisms and processes that are typically considered.

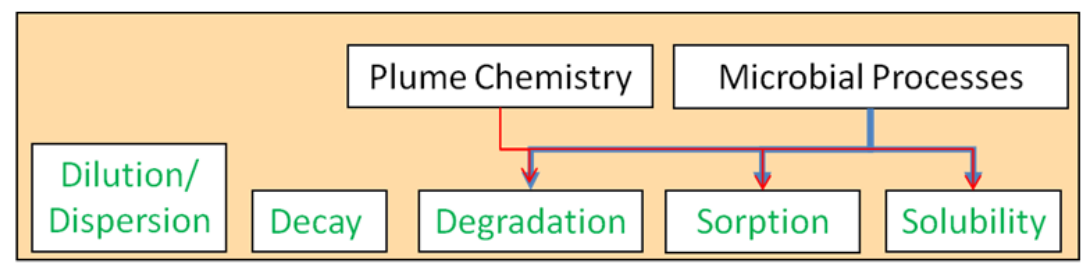

Figure 7. Attenuation mechanisms (green font) for inorganic contaminants in groundwater and factors that can impact attenuation (black font) (adapted from Truex et al. 2011a).

For the vadose zone, there are two major components to attenuation: 1) hydrologic factors that limit water and contaminant flux to the groundwater and 2) hydrobiogeochemical phenomena such as sorption, solubility, degradation/decay, and/or dispersive processes that slow contaminant movement relative to water movement. Figure 8 illustrates the type of mechanisms that may need to be considered for natural attenuation in the vadose zone. Note that Figure 8 includes waste fluid properties and chemistry because, typically, wastes are directly released to the vadose zone and attenuation may be impacted by the nature of the waste material. Also, in comparison to most groundwater situations, there are additional hydraulic processes in the vadose zone that can significantly affect water flow and may have a profound impact on contaminant attenuation. In this case, the Tier II assessment for the vadose zone is "Determine the mechanisms and rates of water and contaminant flux reduction in the vadose zone." 


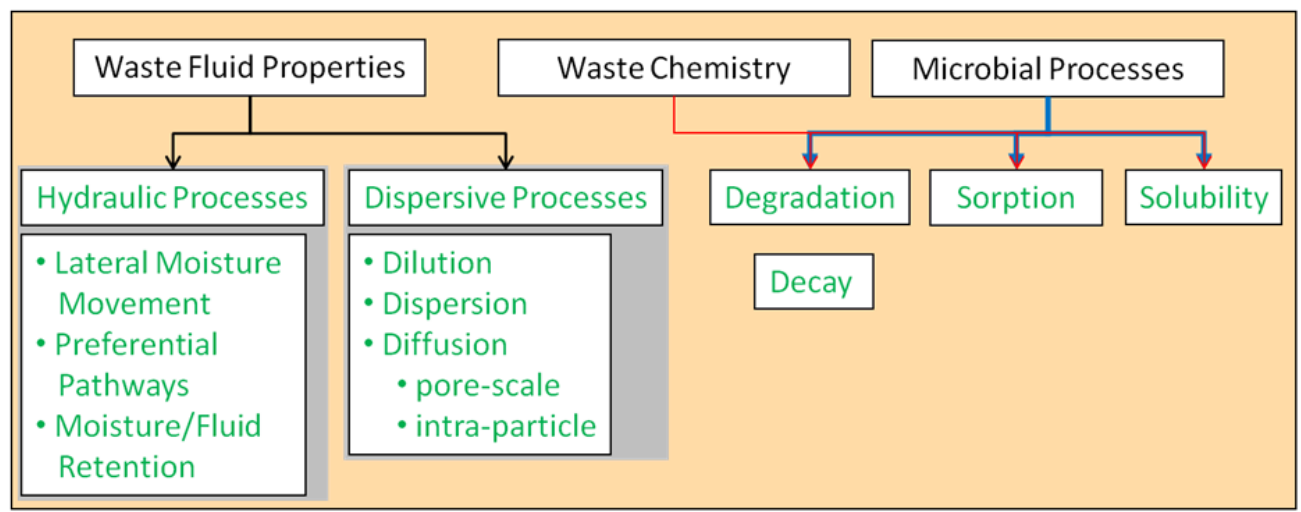

Figure 8. Attenuation mechanisms (green font) for inorganic contaminants in the vadose zone and factors that can impact attenuation (black font).

\section{Tier II Approaches}

To address the Tier II evaluation, a suitable conceptual model of the vadose zone is needed to identify important features and processes for the site in a way that supports estimating their impact on contaminant flux through the vadose zone. At complex sites, the conceptual model would inform use of a numerical model to provide fate and transport estimates incorporating the relevant and important features and processes. The conceptual model is an important technical basis for numerical model configuration and to support assumptions or sensitivities that need to be examined. Key categories of information include vertical stratigraphy, vertical moisture content distribution (e.g., neutron logs), and estimates of lateral spreading for discharged water and contaminants, sediment hydraulic property, waste discharge, and for geochemical and sorption processes. Site-specific information or information from other sources relevant to the specific site may be appropriate. The conceptual model can also describe how this information is represented in the transport calculations and support associated generalizations that may be made to facilitate calculations.

Potential approaches for Tier II include the following items:

- Quantify the impact of the hydraulic processes shown in Figure 8 on moisture flux to groundwater:

- Define sediment properties and distribution of hydraulically important features (e.g., layers or lenses) that need to be considered in evaluating moisture conditions.

- Evaluate vadose zone moisture conditions to estimate flux conditions in comparison to rechargedriven moisture flux. For sites closer to recharge-driven moisture flux conditions, use of streamlined transport analyses may be possible (e.g., reliance on use of the recharge rate as the moisture flux for the future).

- Estimate or measure rate of flux decline over time, based on moisture conditions at the site in relation to moisture conditions for recharge-driven conditions.

- Evaluate the impact of waste fluid properties as appropriate. 
- Quantify sorption, solubility, degradation/decay, and dispersive processes, taking into account changes with depth, waste disposal chemistry, and microbial processes:

- Conduct sediment and pore-water chemistry analyses.

- Perform sequential extraction analyses.

- Evaluate biogeochemical evolution with time and depth from initial waste release as appropriate.

- Evaluate the impact of waste fluid properties as appropriate.

- Quantify or estimate moisture and/or contaminant retention and retained contaminant moisture mass as part of a vadose zone mass balance with released waste materials and discharge to groundwater.

- Use attenuation mechanism parameters/measurements to estimate contaminant retardation relative to moisture flux.

\section{Tier III: Demonstrate Effectiveness and Longevity in Meeting Remediation Goals}

The EPA Protocol Tier III evaluation for groundwater plumes focuses on two elements of natural attenuation that are important in demonstrating that MNA can meet remediation goals. First, natural attenuation effectiveness is assessed based on the capacity of an aquifer system to attenuate contaminants. The aquifer system within the zone identified for treatment (e.g., before reaching a compliance location) must be able to attenuate the amount of contaminants that are present and reach targeted concentration goals. Second, natural attenuation longevity in the aquifer is assessed. Because contaminants are left in place, it is necessary to demonstrate that the mechanisms that decrease concentrations below the identified goals will be able to maintain acceptable concentrations in the groundwater over time, under the range of hydraulic and geochemical conditions that are expected. That is, the attenuation mechanisms in the groundwater must be stable and sustainable.

These assessments of natural attenuation capacity and longevity in groundwater are also relevant to the vadose zone. For the vadose zone, demonstrating attenuation capacity and longevity points to the need to estimate the contaminant flux to groundwater over time and demonstrate that this flux will be acceptable over the long term. Determination of the "acceptable" vadose zone flux must be linked to meeting goals identified for groundwater or other receptor/compliance elements for the site. Thus, the extended Tier III assessment for the vadose zone is "Determine the system capacity and longevity of attenuation by quantifying the contaminant flux from the vadose zone to the groundwater over time and demonstrating that this flux will remain acceptable over the long term."

\section{$\underline{\text { Tier III Approaches }}$}

The Tier III assessment for the vadose zone would rely on predictive modeling or calculations based on recharge rate and the measured or expected range of pore-water concentrations to estimate the contaminant flux to groundwater over time. This assessment can be conducted at different stages in site evaluation using different levels of detail appropriate for the required decisions. For an initial assessment of potential groundwater impact, contaminant transport simulations similar to those conducted for the Hanford Site as described in Section 4 may be appropriate. Use of additional information, such as described for the Tier II assessment, to refine estimates of fate and transport can be added as needed to support the technical basis for remedy decisions (e.g., consistent with a graded approach to modeling [DOE 2012]). A conceptual model, lines-of-evidence approach linked to the attenuation mechanisms 
inherent in vadose zone systems provides a structured approach to support the fate and transport assessment and can also be linked to remedy evaluation, in particular for consideration of natural attenuation contributions to the remedy.

Potential approaches for Tier II include the following items:

- Determine an appropriate recharge rate for use in the site-specific fate and transport analysis and whether changes over time should be considered.

- Estimate the contaminant flux to groundwater as a function of time.

- Conduct a mass balance of retained and mobile contaminants as appropriate to estimate total contaminant mass that will be discharged to the groundwater.

- Consider changes over time that can impact the contaminant flux to groundwater and resultant groundwater plume:

- changes in recharge rate

- changes in pore-water chemistry that impact attenuation

- changes in groundwater elevation or flow rate

- changes induced by enhanced attenuation or active remedies in the vadose zone.

\section{Tier IV: Design a Monitoring Approach}

The fourth tier of the EPA Protocol for groundwater plumes specifies designing a monitoring program using a network of wells to 1) provide adequate areal and vertical coverage to verify that the groundwater plume remains static or shrinks and 2) monitor groundwater chemistry necessary to ensure that attenuation mechanisms are being sustained. This overall approach for groundwater is also relevant to the vadose zone in that it points to the need for verifying attenuation capacity and longevity, although the specific monitoring approach for groundwater using monitoring wells and groundwater chemistry assessment is not directly applicable in the vadose zone. A key approach is to monitor multiple elements identified in the conceptual site model as important to assessing contaminant fate and transport within the system. These "lines of evidence" can be used to verify remedy performance by providing near-term data on natural attenuation processes (capacity) and facilitate transitioning to long-term monitoring strategies that are focused on demonstrating continued compliance with remediation goals and longevity of attenuation (i.e., a progressive systems-based conceptual model approach [Bunn et al. 2012]). For MNA, monitoring needs to address the objectives specified within the "Implementation" section of the EPA OSWER Directive 9200.4-17P, "Use of Monitored Natural Attenuation at Superfund, RCRA Corrective Action, and Underground Storage Tank Sites" (EPA 1999). These implementation objectives are extensions of the proscribed MNA remedy evaluation approach that is strongly based on conceptual model development and establishing appropriate attenuation process knowledge (e.g., Tiers I, II, and III).

\section{$\underline{\text { Tier IV Categories of Vadose Zone Monitoring Approaches }}$}

Developing the monitoring program based on a conceptual model of the site developed through Tiers II and III can help focus the monitoring effort. For instance, only a limited set of data to verify recharge conditions or moisture content of key features in the vadose zone may be sufficient to demonstrate that the expected contaminant flux conditions are being maintained. Infrequent monitoring 
of relatively slow vadose zone transport may be appropriate. If moisture conditions along a vertical profile including key features in the vadose zone were needed, provision of an access borehole with infrequent neutron logging may provide sufficient data to verify contaminant flux conditions. Recharge rate is likely to be a key element of long-term contaminant flux, and monitoring of conditions related to recharge (e.g., surface vegetation/infiltration barriers, annual precipitation) may be sufficient to demonstrate that contaminant flux conditions are within an acceptable range. Some categories of potential monitoring approaches are listed below. Geophysical techniques may also provide a way to evaluate contaminant movement over time, for instance, based on changes in the volumetric distribution of electrical conductivity or moisture over time (e.g., through surface or cross-hole electrical resistivity tomography that can be operated autonomously or with infrequent manual surveys). Using the conceptual model basis and information compiled through Tiers II and III should be considered in developing a monitoring program commensurate with the risk of groundwater impact. Thus, some vadose zone sites may require no or minimal site-specific monitoring and rely more on overall system conditions such as precipitation and vegetative cover that relate to recharge rates. The more site-specific monitoring approaches discussed in this section could be considered for waste sites with more potential risk to groundwater or where remedies are implemented.

Potential approaches for Tier II include the following items:

- Monitor lines of evidence verifying attenuation and fate and transport processes identified in Tiers II and III (e.g., the refined conceptual site model):

- moisture and contaminant flux monitoring, including consideration of

$\circ$ changes at controlling features that are indicators of overall moisture or contaminant flux

$\circ$ quantifying recharge rate and changes over time

- moisture/pressure distribution monitoring as an indicator of moisture flux or retention

- contaminant distribution monitoring over time as an indicator of contaminant flux.

- Monitor geochemical conditions related to stability of sorption, solubility, and/or degradation phenomena.

- Monitor changes in groundwater conditions and enhanced attenuation or active remedy impacts relevant to vadose zone attenuation and impact to groundwater.

- Transition to long-term monitoring configurations that build from the near-term lines-of-evidence monitoring data and use diagnostic elements of the overall environmental system and/or threshold monitoring approaches to demonstrate compliance in a streamlined approach that can be sustained over long durations.

\subsection{Remedy Evaluation Framework: Evaluation Approach}

Concepts based on vadose zone transport characteristics (Section 2.1) and lines-of-evidence, systemsbased, conceptual model approaches and other key elements of MNA (Section 2.2) were compiled and incorporated into a remedy evaluation framework for inorganic, non-volatile contaminants in the vadose zone for use with a target of mitigating contaminant flux to groundwater. The approach is outlined below along with references to key materials presented in Sections 2.1 and 2.2. Section 3 provides additional background on vadose zone transport concepts that provide technical support for this approach. 
Discussion of case study applications of the approach is provided in Section 4. Potentially appropriate characterization and monitoring compatible with this approach are discussed in Section 5.

Evaluation of contaminants in the vadose zone for inorganic, non-volatile contaminants should include the following elements with the goal of developing a conceptual model suitable to support the fate and transport assessment, baseline risk assessment, and remedy decisions, accounting for the natural attenuation processes in the vadose zone (which, in many cases, are expected to significantly impact contaminant flux to groundwater). The fate and transport assessment, with technical support through the elements of the conceptual model, is applied to estimate the temporal profile of contaminant flux to groundwater, incorporating the important vadose zone features and/or processes that control this flux and identifying the location and magnitude of remedial actions that can be applied to augment natural attenuation, if needed, to mitigate the flux and meet groundwater remediation goals. This approach follows the process of developing a conceptual model suitable for supporting projections of future flux to groundwater because it is likely that, in many cases, the baseline risk assessment and remediation decisions for the vadose zone will need to be made all or in part based on projected impacts to groundwater.

- Use the tiered assessment approach as summarized in Figure 6 (Section 2.2) to develop a refined conceptual model of the site. This structured process is intended to provide a suitable technical basis for quantifying the contribution of natural attenuation and making quantitative estimates of contaminant flux to groundwater. The material in Section 2.1 can be used to provide qualitative and quantitative support for developing projections for the temporal profile of contaminant flux to groundwater. A key element is in developing information about the mechanisms listed in Figure 8 (Section 2.2), including the potential waste chemistry considerations described in Section 2.1.4.

- Based on the projected contaminant flux to groundwater, assess the potential resulting concentrations in relation to groundwater remediation goals. The temporal profile of the flux from the vadose zone is important to consider in this assessment because it may result in a finite pulse of contaminants to groundwater at concentrations for concern. Depending on the nature of the contaminant pulse from the vadose zone and the timing of this pulse, natural attenuation or limited active remediation in the groundwater may be appropriate to consider. For situations where the flux of contaminants from the vadose zone is expected to cause longer-duration contamination and/or will be delayed beyond a reasonable implementation period for groundwater actions, other management strategies or vadose zone remediation can be evaluated to identify actions that mitigate the flux through the vadose zone. The information gathered in the tiered assessment approach (Section 2.2) and associated flux estimates can provide a basis to identify effective targets for vadose zone remediation (e.g., chemical or physical flux reduction approaches) that enhance attenuation processes in the vadose zone. Enhancements may include actions that functionally 1) limit fluid flow (e.g., surface or subsurface recharge) through the contaminated zone; 2) change the amount, movement, and/or distribution of subsurface moisture; 3 ) change the properties of the contaminant or subsurface to increase contaminant/sediment interactions and slow contaminant transport; and/or, 4) remove a portion of the contaminant mass such that the remaining contaminant flux is low enough to meet groundwater remediation goals. 


\subsection{Vadose Zone Transport Concepts}

This section presents a brief description of vadose zone transport processes and highlights differences between processes controlling transport in the vadose zone compared to groundwater systems. These differences in transport processes impact contaminant attenuation behavior and need to be considered/ evaluated during the remedy evaluation process described in Section 2. In particular for the unsaturated vadose zone, physical transport processes including advection, dispersion, and diffusion are critical to understanding contaminant transport in the vadose zone, primarily because 1) there is a nonlinear relationship between water content and hydraulic conductivity that complicates water and contaminant flux and 2) subsurface interfaces can dramatically impact vertical moisture and contaminant movement. In addition, the nature of recharge at the water table and mixing of recharge water with groundwater are important relative to the resultant groundwater contaminant concentrations. There is significant information about relevant biogeochemical processes due to their dominant roles in contaminant attenuation in groundwater systems, but these processes must be evaluated in the context of the vadose zone properties, especially due to the presence of a gas phase.

\subsection{Physical Transport Processes and Controlling Features}

The physical processes controlling fluid flow in the vadose zone are quite different from those within the groundwater system. In addition, the transport attenuation processes are generally more significant in the vadose zone compared to the groundwater system. The nonlinear relationship between water content and hydraulic conductivity must be considered in evaluating flow and transport through the vadose zone. Ultimately, a primary driving force for long-term transport is often the net recharge at ground surface, a function of precipitation, infiltration, and transpiration processes. Subsurface interfaces that separate zones of different particle size distributions and properties (i.e., layering) are also important due to the impact on unsaturated flow processes. Preferred flow pathways can occur at interfaces and may be important in the overall rate of vertical contaminant movement (e.g., dipping layers, vertical features, or fractures). Smaller-scale interfaces may also be important relative to intra- or inter-particle contaminant diffusion and the relative mobility of water and contaminants within different micro-scale regimes of the subsurface. Specific features of vadose zone moisture transport are discussed in the following sections.

\subsubsection{Moisture Retention, Capillary Trapping, and Gravity Drainage}

The most obvious difference between the vadose zone and groundwater is that the vadose zone is unsaturated with respect to water. The groundwater system pore space is completely filled with water, and there is generally no gas present as a separate phase. Above the capillary fringe in the vadose zone, there is some gas (i.e., air) present, and the pore space is not filled with water.

Capillary forces act on pore water in the vadose zone and impact fluid flow and water content distributions. Capillary pressure causes porous media to wick in the wetting fluid and displace the nonwetting fluid (Cohen and Mercer 1990; Hillel 1998). Water is generally wetting, and air is generally nonwetting. The capillary pressure is also commonly referred to as suction or tension and converted to matric potential (in terms of head). 
This relationship of capillary pressure, or matric potential, versus water content is termed the moisture retention curve (Figure 9). The capillary pressure generally increases nonlinearly with decreasing water content. Alternatively, capillarity generally increases with decreasing pore radius (or decreasing grain size). Thus, the largest pores drain first and water contents are generally higher for finer-grained materials. The last water to drain comes from the smallest pores.

The dependence of the water content on the capillary pressure is a characteristic property of a soil (Figure 9). General properties of the characteristic relationship can be determined based on soil type alone. For example, the air-entry pressure is dependent on the largest pore size, which is proportional to the grain size. Furthermore, the smoothness of the curve is related to the distribution of pore sizes; a narrow range of pore diameters leads to a step change in saturation. The residual saturation is generally higher for finer-grained materials.

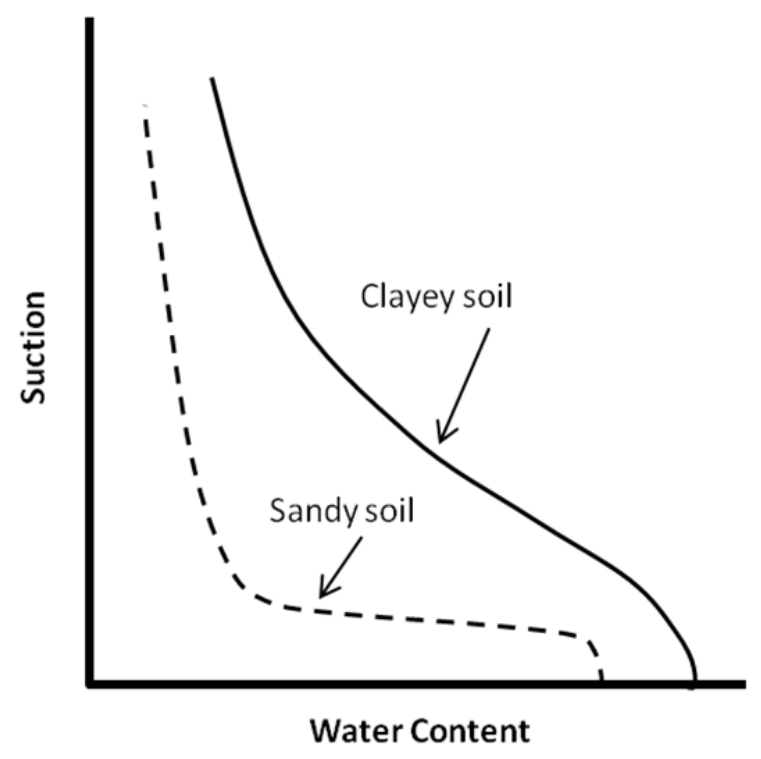

Figure 9. Illustrative soil characteristic curves for a fine (clayey) soil and a coarse (sandy) soil.

This moisture retention relationship also varies depending on the direction and history (hysteresis) of change in water content, which will increase (wetting) during increased recharge/infiltration or decrease (drainage) after increased recharge/infiltration or during increased evaporation or drought. Hysteresis is known to occur in multiphase immiscible systems in intermediate saturation ranges, which complicates the prediction of constitutive relationships (Kechavarzi et al. 2005). Hysteresis is created by a combination of pore processes including saturation of variable pore sizes, saturation-dependent residual saturations, and non-wetting fluid entrapment (Lenhard 1992; Lenhard et al. 2004).

Another force controlling unsaturated water content is gravity. Gravity works to induce vertical migration of water, but the rate of movement is a function of both gravity and capillary forces. For instance, accompanying a significant pulse of water infiltration (waste discharge), water content can be elevated near the surface such that there is water in larger pores where capillary forces are weaker and vertical movement (drainage) due to gravity can be rapid. However, as the pulse of water moves through into the vadose zone, retention of water along the flow path reduces the amount of water that can fill larger pores and capillary forces begin to become significant with respect to control of water movement. 
With further vertical and lateral movement, water content will continue to decrease and will tend toward conditions in the vadose zone where movement is controlled by the recharge rate (e.g., steady-state conditions).

\subsubsection{Darcy's Law, Hydraulic Conductivity, and Relative Permeability}

Groundwater systems are water saturated (contain no gas phase), and thus only liquid water is available for fluid flow. For groundwater systems, the Darcy flux $[\mathrm{L} / \mathrm{T}]$ is a function of the hydraulic conductivity $[\mathrm{L} / \mathrm{T}]$ and the hydraulic gradient $[\mathrm{L} / \mathrm{L}]$ (Darcy 1856). Water movement in the vadose zone can also be described with a version of Darcy's Law that was modified by Buckingham (1907). The driving force for infiltration flux is the hydraulic head gradient, which can be separated into the matric potential head gradient and the gravity unit gradient. However, the primary difference for Darcy's Law in the vadose zone is that the hydraulic conductivity is not a constant for a given porous medium. The unsaturated hydraulic conductivity is highly variable as a function of the water content, which varies depending on the distance from the water table or with changes in grain size and permeability. Generally, as water content decreases, the volume of water and the cross-sectional area within pores available for flow decreases, which restricts water permeability and water flow, similar to decreasing the pore size from a coarse to a more-fine grained material. The relative water permeability of a given soil can change over many orders of magnitude with changes in saturation. These processes control fluid and contaminant flux in the vadose zone and can act as attenuation processes for infiltrating water and contaminant mass.

\subsubsection{Unsaturated Infiltration and Recharge}

Recharge is when infiltrating water is released from the vadose zone and mixes with the groundwater system. All precipitation may not infiltrate (e.g., surface run-off), and all infiltrated water may not recharge the groundwater system (e.g., due mainly to evapotranspiration and vadose zone storage). The infiltration rate depends on water application rate at the surface, capillary pressure distribution in the subsurface, and water-relative permeability. In some cases, water ponding at the surface may occur.

Most waste discharges are associated with a temporary leak or release, which is terminated at some point. As mentioned above, the water saturation increases during the additional infiltration period, but then drainage occurs, decreasing the water content and infiltration rates. After the termination of water release, the elevated saturation dissipates rapidly during drainage where the added water is wicked laterally and vertically as a function of gravitational and capillary forces (Figure 10).

Increased infiltration flux rates due to temporary releases are short-lived transient behaviors, and infiltration rates decay exponentially until they reach the long-term, natural recharge rate of infiltration. As water content decreases during this drainage process (Figure 10), the relative ratio of capillary to gravity force increases (Figure 9), which induces more lateral flow and increases retention and immobilization of water within vadose-zone pores. This restriction to vertical flow with decreasing water content during drainage is also enhanced by the nonlinear decreases in hydraulic conductivity. Transitions in material permeability, especially horizontal layering (e.g., numerous sedimentary deposits), typically enhance these restrictions to vertical flow and induce increased lateral flow, which further reduces water content during drainage. 


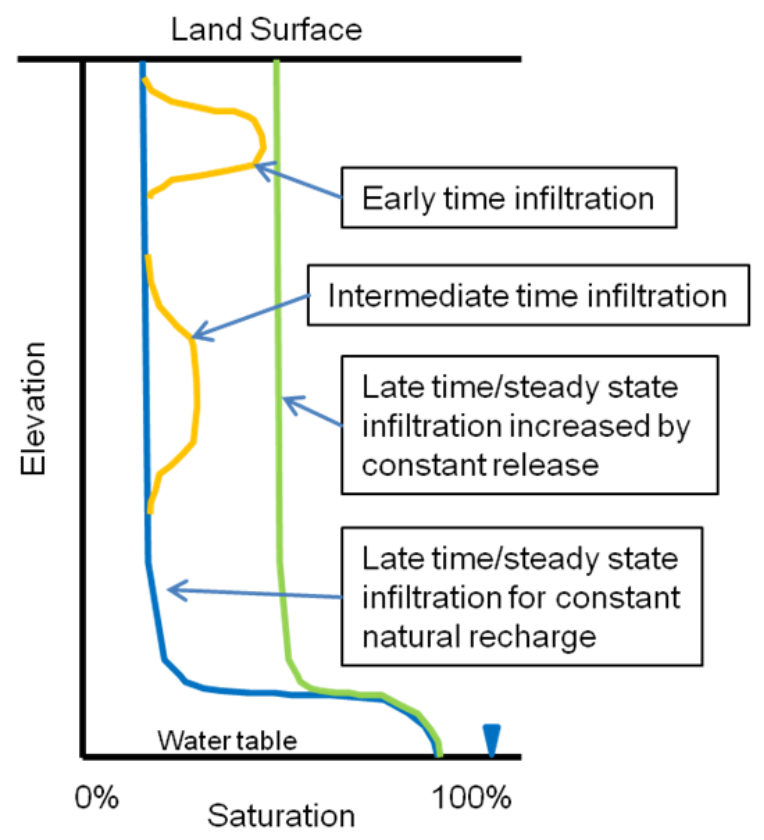

Figure 10. Illustrative plot of water saturation versus depth below land surface for both transient and steady-state infiltration cases. The blue and green lines indicate steady conditions for natural, constant recharge and anomalously increased, constant infiltration rates, respectively. The orange lines indicate the transient water content increase due to a temporary release of water and subsequent decline and dispersal during drainage.

Infiltrated water released into the vadose zone will migrate through the vadose zone vertically and mix into the groundwater. However, if the storage capacity and size of the vadose zone are large compared to the amount of water released, the water will be wicked into storage and trapped by capillary forces as the water saturation profile decreases (Figure 2 and Figure 10). The size of the vadose zone, its capacity for water storage, and its tendency to spread/disperse infiltrating flow rates (i.e., water flux) control the water and contaminant attenuation behavior and capacity. If these processes are quantified, they can be compared to release quantities to determine how much attenuation of the waste release will occur in the vadose zone (Figure 1). This evaluation then supports consideration of MNA as a remedy and any subsequent evaluations of other or additional remedies that might be required. Figure 1A illustrates a case where attenuation processes in the vadose zone partially mitigate the flux of an introduced pulse to groundwater. Figure 1B illustrates the case where vadose zone attenuation is more significant and flux to the groundwater remains low.

Significant quantities of water and contamination have been discharged and migrated into a thick vadose zone at the U.S. Department of Energy (DOE) Hanford Site. Analyses and data from this site provide information useful to examine vadose zone transport processes (see also Section 4). For instance, simulations conducted examining moisture changes at the Hanford 200 Area 216-U-10 site (U Pond) are an illustrative example of how added water pulses to the vadose zone dissipate over time. Oostrom et al. (2008) conducted modeling investigations to predict carbon tetrachloride contamination distribution and transport behavior at the Hanford 216-Z-9 waste site. In addition to and associated with the carbon tetrachloride modeling, this study also simulated large-scale infiltration and drainage that occurred in the area from 1954 to 2005 . 
A vast amount of water $\left(1.628 \times 10^{11} \mathrm{~L}\right)$ was released at the U Pond site from 1944 to 1985 . Simulations of this release and subsequent drainage after 1985 were conducted. The natural recharge water flux was assumed to be $0.5 \mathrm{~cm} / \mathrm{yr}$, which was applied for a steady-state pre-modeling simulation to obtain the initial water content distribution. The modeled compared water saturations at different times to the initial (1944) water-saturation conditions along a cross section of the vadose zone. Figure 11 shows the simulated conditions for 1985, at the end of water releases to the U Pond, and Figure 12 illustrates the conditions for 2007. These simulation results illustrate the magnitude of lateral spreading during releases and the return to lower moisture content conditions that approach recharge-driven conditions after discharges cease.

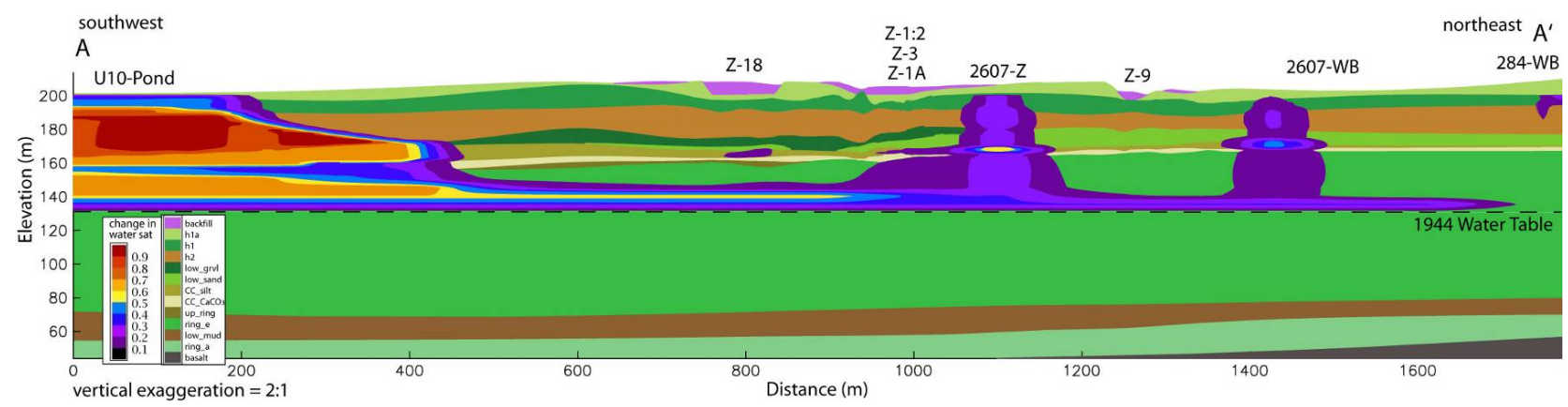

Figure 11. Differences in water saturation between 1985 and 1944 (initial) (Oostrom et al. 2008).

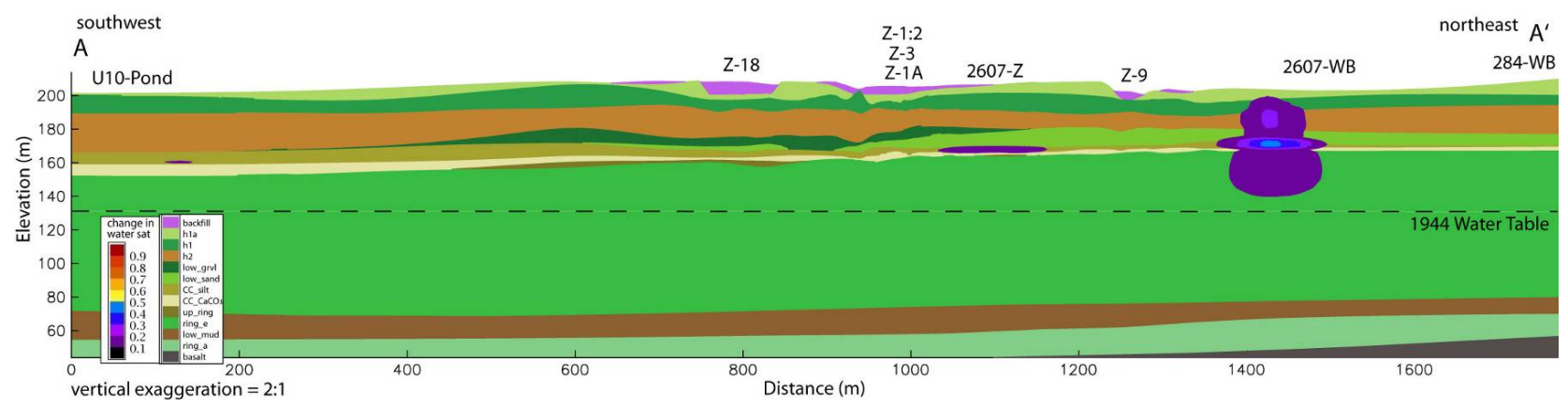

Figure 12. Differences in water saturation between 2007 and 1944 (initial) (Oostrom et al. 2008).

\subsubsection{Heterogeneous Permeability Impacts on Infiltration}

There are significant implications for the vadose zone's variable hydraulic conductivity that are relevant for the attenuation of the transport of water and contaminants. Generally, for groundwater systems we consider coarse-grained materials (higher permeability) to have much higher hydraulic conductivities compared to fine-grained materials (lower permeability) for groundwater systems. However, capillary pressure holds water more tightly in fine-grained materials. So, the largest pores drain first, which may be the majority of the pores within coarse-grained materials. In the vadose zone, at the same capillary pressure, fine-grained sediment may have higher water content and a higher unsaturated hydraulic conductivity than coarse-grained material.

The presence of low-permeability zones, or layers, acts to mitigate the magnitude of the infiltration rate, which is an attenuation process. The lower-permeability layers, even relatively thin ones, will become a dominant control over the maximum infiltration rate, and they also serve to enhance lateral 
fluid flow in the unsaturated zone. This lateral flow is important as an attenuation process for several reasons. First, increases in lateral flow equate to decreases in vertical flow toward the water table. Additionally, lateral migration increases the volume of vadose zone impacted and the amount of water that can be stored and trapped by capillary forces. The additional volume of vadose zone increases the potential for geochemical retention processes (e.g., adsorption) to immobilize contaminants. Finally, lateral flow increases the mixing of contaminated water with uncontaminated water, which attenuates concentrations and mass flux through dilution, diffusion, and dispersion.

Three special cases for layered or heterogeneous permeability or grain-size distributions have relevance for contaminant attenuation processes in the vadose zone. These include perched water tables, capillary barriers, and preferential flow systems. Each of these cases is described below with relevance to contaminant attenuation.

Perched water tables occur in the vadose zone when infiltrating water reaches a fine-grained, lowerpermeability material underlying a coarser, higher-permeability material. This causes the flux to decrease due to the lower hydraulic conductivity of the fine-grained material until enough water collects to create ponding and saturation of the overlying higher-permeability material. This may induce an increased saturation and pressure buildup in the overlying higher-permeability material that may also induce lateral flow, which along with the reduced vertical flux are both contaminant attenuation processes.

Similarly, a capillary barrier involves principles of unsaturated flow between soils of different textures and permeabilities (Rasmuson and Eriksson 1988). Capillary barriers occur when infiltrating water reaches a coarser, higher-permeability material underlying a finer-grained, lower-permeability material. At initially gravity-drained conditions, the coarse material has a lower water content and lower unsaturated hydraulic conductivity than the overlying finer-grained material. The air is trapped in the coarse material, and the water is wicked in the finer material due to capillarity; this force must be exceeded to allow flow into the underlying coarser material. This causes the flux to decrease, or essentially stop, due to the lower hydraulic conductivity of the coarser-grained material until enough water collects in the overlying materials to exceed the air-entry pressure of the coarser-grained material. Therefore, capillary barriers are caused by differences in physical properties of soil textures and can occur naturally or are man-made. These capillary barriers are used for various landfill or mine waste material covers to inhibit the infiltration of water vertically. If they are naturally present in the vadose zone, they may contribute significantly to the attenuation of infiltrating water and contaminant mass flux vertically toward the groundwater. As water and pressure build up above the capillary barrier, lateral flow is induced. Both the vertical flux reduction and induced lateral flow are contaminant attenuation processes.

Preferential flow may occur in the vadose zone due to heterogeneous permeability features or simply due to the physics of the infiltrating solution. Unstable flow features (fingers or preferential flow paths) may occur in unsaturated infiltration due to the increased density of the overlying water relative to the underlying air that is being displaced (Glass et al. 1989a, 1989b, 1989c, 1989d, 1990, 1991; Parlange et al. 1990). The formation and extent of these unstable flow features may be more exacerbated by higher density and higher viscosity infiltrating waste solutions. In addition, vertical or steeply dipping features of elevated hydraulic conductivity may cause preferential flow. These preferential flow behaviors may increase the rate of infiltration locally near these features while the water content is elevated. Alternatively, preferential flow can act as a form of contaminant attenuation if contaminants become retained within zones that are not part of these preferential flow paths. 


\subsubsection{Solute Transport}

Solute transport is affected by the advection, diffusion, and dispersion processes in addition to the potential biogeochemical processes discussed in Section 3.2.

\subsubsection{Advection}

The bulk movement of solutes due to, and associated with, flowing pore water is known as advection (Freeze and Cherry 1979). Given that transport by advection is the movement of solutes along with flowing groundwater, the driving force for advection is the hydraulic head gradient. The rate of advection (pore-water velocity) is directly dependent on the saturated porosity and hydraulic conductivity, which both decrease with decreasing water content, as described above. The rate of advection, along with the rate of infiltration/recharge, can decrease by orders of magnitude with the unsaturated hydraulic conductivity and is typically much lower than rates observed in groundwater systems. Additional advective transport behaviors may occur depending on the ionic strength and density of the waste solution that affect the pore-water behavior. Preferential flow features may impact the spatial distribution and rate of advection. Typically, vadose zone fluid flow and associated solute advection is considered to be dominated by gravity-driven vertical flow. However, various unsaturated flow processes may change the relative amount of vertical and lateral advection.

\subsubsection{Diffusion Processes}

The movement of solutes due to random molecular motion is known as molecular diffusion (Freeze and Cherry 1979). Diffusion is commonly described using Fick's Law where the flux of solutes due to diffusion is proportional to the solute concentration gradient. Elevated solute concentrations in waste solutions that contact native pore water may cause concentration gradients and lead to diffusional movement of solutes that disperses solutes and lowers concentrations. Diffusion within variably saturated vadose zone systems can be influenced by changes in water content. Generally, the rate of solute mass transport by diffusion is lower at lower water content, primarily attributed to increased tortuosity and reduced cross-sectional area available for mass transport at lower water content (Hu and Wang 2003).

\subsubsection{Dispersion Processes}

Dispersion is a physical mass transport process that has been described through analogy to diffusion due to the tendency for both processes to create solute transport through the spreading out of solute plumes (Freeze and Cherry 1979). One significant difference is that diffusion occurs at the molecular scale and dispersion occurs at larger scales and is related to pore-water flow conditions. The inter- and intra-pore velocity variations create variability in travel time, which smears out the concentration distribution. The same behavior is observed in heterogeneous layered sediments when differences in permeability cause transport velocity and travel time variations. The main phenomena that are responsible for dispersion include

1. microscopic: axial diffusion (concentration gradient); mechanical mixing (pore size variation); boundary layer mass transfer (dead-end pores) 
2. macroscopic: structured porous media (aggregation, fractures, macropores); tortuosity (relative magnitude of solute transport path length convolution)

3. megascopic: heterogeneous porous media (hydraulic conductivity variability).

In the vadose zone, as water infiltrates, it may displace and/or mix with the antecedent (uncontaminated) pore water at the leading edge of the infiltrating contaminant plume. Additionally, after the release of contaminant solution has ceased, natural recharge will continue with water that is presumably contaminant free, which will mix with the resident contaminated pore water. In both of these cases, contaminant concentrations will be diluted through mixing with lower concentration, or clean, water, which acts as an attenuation process.

According to capillary theory, as a medium desaturates, the largest pores are the first to dewater (Freeze and Cherry 1979). These pores have the largest intrapore velocity variations, which would suggest that dispersivity decreases with decreasing water content. Additionally, as the water content decreases from complete saturation, the variance of pore sizes that continue to conduct water decreases, which may also cause dispersivity to decrease. Based on this reasoning, the longitudinal dispersivity should decrease with decreasing water content. However, tortuosity may also have a significant role in changes in dispersivity with decreasing water content. Water must migrate around pore spaces containing air, and as fewer pores are water filled, the water-filled pathway will become more tortuous. This behavior would tend to increase lateral spreading and the magnitude of dispersivity. Recently, researchers have presented data that suggests a trend of decreasing dispersivity with increasing water content (Padilla et al. 1999; Toride et al. 2003; Mayer et al. 2008). However, our mechanistic understanding of processes controlling the changes in dispersivity with water content variations is still lacking, and few, if any, investigations have examined heterogeneities, spatially variable water saturations, or impacts of unsaturated flow hysteresis.

\subsubsection{Dispersion Due to Mobile/Immobile Zone Mass Transfer}

As opposed to axial or bulk-fluid phase diffusion, diffusive mass transfer from mobile pore water into and out of immobile pore water (e.g., water in dead-end pore space) is generally considered physical nonequilibrium transport (Brusseau et al. 1989). Retention and rate-limited delays for solute transport resulting from the diffusive mass transfer between mobile and immobile pores are analogous to delays in transport due to adsorption (Brusseau et al. 1989, 1993). The majority of research evaluating physical nonequilibrium has considered only water-saturated systems. Recently, researchers have presented data that suggest significant variability in mobile to immobile pore-space fractions with changes in water content (Padilla et al. 1999; Toride et al. 2003; Mayer et al. 2008). The vadose zone water retention at the pore scale may include water retained in dead-end pores or water that is not connected with the main drainage pathways.

\subsection{Biogeochemical Processes}

Geochemical processes and biological processes can have a significant impact on contaminant transport and attenuation processes in the subsurface. In fact, these are considered to be of most significant importance for groundwater systems. As such, the existing MNA guidance, including the EPA and Interstate Technology \& Regulatory Council (ITRC) protocols (EPA 2007a, 2007b, 2010; ITRC 2010) and the Scenarios document (Truex et al. 2011a), discuss these processes in detail. The following 
sections briefly introduce these processes with a focus on the relevance to the vadose zone and the differences between the behaviors in the vadose zone compared to the groundwater systems.

\subsubsection{Geochemical Considerations}

In contrast to many groundwater systems, geochemical considerations in the vadose zone must include the presence of a gas phase, potential for "extreme" chemistry due to waste solution characteristics (e.g., low or high $\mathrm{pH}$, ionic strength, and others), and the potential for long residence time in the vadose zone. In particular, presence of the gas phase may lead to exchange of oxygen and associated impact on the redox conditions of the pore water and exchange of other chemicals including carbon dioxide that may impact geochemical reactions of relevance to contaminant transport.

\subsubsection{Considerations for Biologically Mediated Processes}

Microbial activity is influenced by the water potential with generally decreasing activity with decreasing water content (Soil Science Society of America 1981). The two key factors in the vadose zone with respect to microbial activity are the matric potential and the osmotic potential. Thus, evaluation of biologically mediated processes in the vadose zone must consider the moisture content and associated matric potential and the solute concentration (osmotic potential). Geochemical conditions also impact microbial activity. Key factors include the $\mathrm{pH}$, buffering capacity, oxidation-reduction potential, and the presence of dissolved or solid-phase electron acceptors. Thus, presence of a gas phase and the associated impact on pore-water geochemistry need to be considered in evaluating microbial activity in the vadose zone.

\subsection{Potential Applications and Case Study Examples}

One application for the assessment of MNA for inorganic contaminants within the vadose zone is the Central Plateau of the DOE Hanford Site (DOE 2010). The remediation efforts being developed for the deep vadose zone at the Central Plateau are central to the overall Hanford cleanup because the deep vadose zone contamination represents a potential ongoing source of contamination to the underlying aquifer and the Columbia River (DOE 2010).

Currently, the largest inventory of subsurface contamination across the Hanford Site resides beneath the Central Plateau (DOE 2010). The Central Plateau of the Hanford Site contains more than 800 wastedisposal sites (e.g., ponds, ditches, cribs, trenches, reverse wells, and landfills) contaminated with radioactive and other hazardous contaminants. The majority of these sites were used for disposal of liquid waste generated during reprocessing of spent uranium fuel to recover plutonium.

Groundwater remediation is being implemented for several of the groundwater contamination plumes. However, a significant amount of contamination remains in the vadose zone beneath the Central Plateau and may impact groundwater in the future (Gee et al. 2007). The potential attenuation of contaminants within the vadose zone is important for fate and transport assessment and evaluating protection of groundwater at these sites. As described in the remedy evaluation framework herein, attenuation-based analyses are expected to be important in supporting effective remedy selection. 
For the remedy evaluation framework, evaluation is conducted in the context of determining potential impact of vadose zone contamination on groundwater quality. Previously, Fayer et al. (2010) summarized the major data collection and modeling efforts that are available for Hanford Site-wide evaluation of contamination and need for remediation. In addition, Eslinger et al. (2006) developed a suite of interrelated computer codes, termed the System Assessment Capability (SAC), and used the SAC to conduct a series of risk analyses for nearly all of the Central Plateau waste sites (410 sites were included).

The SAC considered the source term, waste release, vadose zone, groundwater, air, and the Columbia River, and it supports both deterministic and stochastic fate and transport models of radioactive and chemical contaminants in the environment and the associated human and ecological impacts. For inventory, the SAC considered liquid discharges, solid waste burials, and unplanned releases, as well as some other releases. This study did not address the residuals in the single-shell or double-shell tanks. The SAC models are capable of multidimensional simulations, but for reasons particular to this study, the vadose zone calculations were conducted in one dimension, in which the size of the wetted area was scaled based on maximum liquid discharge rates and minimum saturated hydraulic conductivity of the vadose zone lithologies. Because all vadose simulations were conducted in one dimension, lateral flow induced by heterogeneities in sediment properties and geologic lithologies was not explicitly addressed.

Individual site assessment included flow and transport simulation within the vadose zone linked to groundwater impact through a mixing model approach. Contaminant concentrations and flow/flux rates were determined over time for the vadose zone at each site. Then the contaminant-released activity or mass/concentration from the vadose zone to the groundwater was converted to a hypothetical groundwater concentration in the aquifer using a "dilution factor" approach as defined in the state of Washington's Model Toxics Control Act (WAC 173-340). The dilution factor from vadose zone to groundwater concentration is equal to the sum of the groundwater and vadose zone infiltration flow rates divided by the vadose zone infiltration flow rate. The infiltration rates were output from the SAC modeling, and the groundwater velocity results were derived from published groundwater model results (Thorne et al. 2006). The output of the SAC analyses used three time periods (2006-2100, 2101-3100, and 3101-12000 date ranges) to quantify predicted releases of contaminants (uranium, I-129, and Tc-99) from the vadose zone to groundwater.

Thus, the SAC evaluation of waste sites is consistent with the vadose zone remedy evaluation and implementation framework (Figure 6), and uses a consistent evaluation approach to predict impact to groundwater and provides a means to prioritize contamination issues and remediation requirements across the entire Central Plateau. The SAC results use information about waste disposal inventory and numerical modeling to predict contaminant impacts to the groundwater. Thus, these results are useful as an initial categorization of sites and identifying relative priority based on predicted impact to groundwater (Table 2).

The SAC results (Eslinger et al. 2006) were used as the root data for the categorization in Table 2. The predicted impact to groundwater at each of the 410 waste sites was evaluated based on the maximum concentration in groundwater divided by the drinking water standard (DWS), which was termed DWS Factor. The listed waste sites were sorted (high to low DWS Factor) for each of the three contaminants for each of the three time ranges. The sites were then classified, with DWS Factor greater than 10 as high impact and sites with DWS Factor ranging from 1 to 10 as medium impact to groundwater. Low-impact sites had a DWS Factor equal to or less than 1 (not included on Table 2) and are candidates for no action 
if supported by a risk assessment (a potential application of the framework). Several of the waste sites contained more than one contaminant and were predicted to have differences in DWS Factor at different times. Table 2 contains all the exceedances for each contaminant at the high- and medium-impact sites. The "DWS Factor" column for a specific high-impact site contains all DWS Factor values greater than 10 at that site (separated by /), and the "Contamination Issue" column for the same site contains the description (in the same order as the DWS Factor column and separated by /) of which contaminants (Tc, I, or $\mathrm{U})$ and time periods $(1,2$, or 3$)$ were predicted to have the DWS Factor values greater than 10 . For example, 241-SX-108 was predicted to have a DWS of 70 for Tc1 (Tc up to 2100), of 43 for Tc2 (Tc up to 3100 ), and of 53 for I2 (I up to 3100). Red text is from groundwater concentration data. For instance, the DWS Factor value of 56 in red and parentheses is an estimate from recent groundwater concentration data (for Tc1 in comparison to the SAC value of 70).

Table 2. Categorization of vadose zone sites based on potential impact to groundwater. Sites with predicted groundwater concentrations less than the DWS are not shown ( $\sim 300$ sites). Contamination issues are in the same order of presentation as the DWS Factor for each site (see text). Contaminant issue is shown as I (I-129), Tc (Tc-99), and U (uranium) for time intervals 1 (up to 2100), 2 (up to 3100), and 3 (up to 12000). Red text shows information added based on recent groundwater plume data.

\begin{tabular}{|c|c|c|c|}
\hline Site Name & Site_ID & DWS Factor & $\begin{array}{l}\text { Contamination Issue } \\
\text { and Time }\end{array}$ \\
\hline \multicolumn{4}{|c|}{$\begin{array}{l}\text { High Potential Impact Sites (Estimated Concentrations Greater than 10X of the Drinking } \\
\text { Water Standard) }\end{array}$} \\
\hline REDOX cribs and trenches & $\begin{array}{l}216-S-7 \\
216-S-1 / 2\end{array}$ & $\begin{array}{l}82(37) / 30 / 11 \\
14\end{array}$ & $\begin{array}{l}\text { I All Times } \\
\text { I3 }\end{array}$ \\
\hline S/SX tank farms \& vicinity & $\begin{array}{l}\text { 241-SX-108 } \\
241-S X-107 \\
241-S X-115 \\
241-S X-104 \\
241-S-104\end{array}$ & $\begin{array}{l}70(56) / 43 / 53 \\
42(56) / 14 / 17 \\
25(56) / 13 / 17 \\
12 \\
22\end{array}$ & $\begin{array}{l}\mathrm{Tc} 1 / \mathrm{Tc} 2 / \mathrm{I} 2 \\
\mathrm{Tc} 1 / \mathrm{Tc} 2 / \mathrm{I} 2 \\
\mathrm{Tc} 1 / \mathrm{Tc} 2 / \mathrm{I} 2 \\
\mathrm{Tc} 2 \\
\mathrm{Tc} 1\end{array}$ \\
\hline $\mathrm{T}$ tank farm \& vicinity & $241-\mathrm{T}-106$ & $64(125) / 104 / 57$ & $\mathrm{Tc} 1 / \mathrm{Tc} 2 / \mathrm{I} 2$ \\
\hline U tank farm & 241-U-104 & 23 & Tc1 \\
\hline Solid Waste - 200-ZP-1 & $\begin{array}{l}218-\mathrm{W}-4 \mathrm{~B} \\
218-\mathrm{W}-5\end{array}$ & $\begin{array}{l}17 \\
12\end{array}$ & $\begin{array}{l}\text { I3 } \\
\text { Tc3 }\end{array}$ \\
\hline $\mathrm{BC}$ cribs/trenches & multiple & $10-17$ & $\mathrm{Tc} 2$ \\
\hline TX/TY tank farms & 241-TX-107 & $23 / 11$ & $\mathrm{I} 1 / \mathrm{I} 2$ \\
\hline B, BX, BY tank farms \& vicinity & BX-102 & $115 / 4 / 34$ & $\mathrm{U} 1 / \mathrm{I} 1 / \mathrm{Tc} 1$ \\
\hline B, BX, BY tank farms \& vicinity & BY-cribs & $14 / 2$ & $\mathrm{Tc} 1 / \mathrm{I} 1$ \\
\hline U cribs & $216-U-1 / 2$ & $12 / 46 / 3$ & $\mathrm{U} 1 / \mathrm{Tc} 1 / \mathrm{I} 1$ \\
\hline B cribs & 216-B-12 & 10 & U1 \\
\hline
\end{tabular}


Table 2. (contd)

\begin{tabular}{|c|c|c|c|}
\hline Site Name & Site_ID & DWS Factor & $\begin{array}{l}\text { Contamination Issue } \\
\text { and Time }\end{array}$ \\
\hline \multicolumn{4}{|c|}{$\begin{array}{l}\text { Medium Potential Impact Sites (Estimated Concentrations Between 1X and 10X of the } \\
\text { Drinking Water Standard) }\end{array}$} \\
\hline S/SX tank farms \& vicinity & $\begin{array}{l}\text { 241-SX-104, } \\
108,109 \\
110,111 \\
112,113\end{array}$ & $1-10$ & $\mathrm{Tc} 1 / \mathrm{Tc} 2 / \mathrm{I} 2 / \mathrm{I} 3$ \\
\hline $\mathrm{U}$ tank farm & $\begin{array}{l}241-U-104 \\
241-U-112 \\
241-U-110 \\
241-U-101\end{array}$ & $\begin{array}{l}2 / 10 / 9 \\
6(3) / 2 / 4 \\
1 / 2 / 2 \\
6 / 2 / 3\end{array}$ & $\begin{array}{l}\mathrm{U} 3 / \mathrm{I} 2 / \mathrm{Tc} 2 \\
\mathrm{Tc} 1 / \mathrm{Tc} 2 / \mathrm{I} 2 \\
\mathrm{Tc} 1 / \mathrm{Tc} 2 / \mathrm{I} 2 \\
\mathrm{Tc} 1 / \mathrm{Tc} 2 / \mathrm{I} 2\end{array}$ \\
\hline TY cribs \& vicinity & $\begin{array}{l}216-\mathrm{T}-26 \\
216-\mathrm{T}-18\end{array}$ & $\begin{array}{l}2 / 8 / 4 / 2 / 2 \\
3\end{array}$ & $\begin{array}{l}\mathrm{Tc} 1 / \mathrm{I} 1 / \mathrm{I} 2 / \mathrm{I} 3 / \mathrm{U} 3 \\
\mathrm{I} 1\end{array}$ \\
\hline REDOX cribs and trenches & $\begin{array}{l}216-\mathrm{S}-1 / 2 \\
216-\mathrm{S}-9 \\
216-\mathrm{S}-7\end{array}$ & $\begin{array}{l}9(37) / 14 / 2 \\
3 \\
2\end{array}$ & $\begin{array}{l}\mathrm{I} 1 / \mathrm{I} 2 / \mathrm{Tc} 1 \\
\mathrm{I} 3 \\
\mathrm{U} 3\end{array}$ \\
\hline $\mathrm{A} / \mathrm{AX}$ tank farms & $\begin{array}{l}241-\mathrm{A}-103 \\
241-\mathrm{AX}-102\end{array}$ & $\begin{array}{l}9 / 7 \\
1(7) / 1\end{array}$ & $\begin{array}{l}\mathrm{Tc} 2 / \mathrm{I} 2 \\
\mathrm{Tc} 1 / \mathrm{Tc} 2\end{array}$ \\
\hline $\mathrm{BC}$ cribs/trenches & multiple & $1-9$ & $\mathrm{Tc} 2 / \mathrm{Tc} 3 / \mathrm{I} 3$ \\
\hline All others in 200-UP-1 & 216-W-LWC & $7 / 1$ & $\mathrm{I} 1 / \mathrm{I} 2$ \\
\hline TX/TY tank farms & $\begin{array}{l}241-\mathrm{TY}-103 \\
241-\mathrm{TX}-107 \\
241-\mathrm{TY}-105 \\
241-\mathrm{TY}-101 \\
241-\mathrm{TY}-106\end{array}$ & $\begin{array}{l}6 / 7 \\
6 \\
5 / 1 / 2 \\
2 / 2 \\
3\end{array}$ & $\begin{array}{l}\text { I2/Tc2 } \\
\text { Tc1 } \\
\text { Tc1/Tc2/I2 } \\
\text { I2/Tc2 } \\
\text { Tc1 }\end{array}$ \\
\hline TX trenches & $\begin{array}{l}216-T-25 \\
216-T-24 \\
216-T-23 \\
216-T-22\end{array}$ & $\begin{array}{l}6 / 2 \\
2 \\
2 \\
2\end{array}$ & $\begin{array}{l}\mathrm{I} 1 / \mathrm{I} 2 \\
\mathrm{I} 2 \\
\mathrm{I} 2 \\
\mathrm{I} 2\end{array}$ \\
\hline PUREX cribs and trenches & $\begin{array}{l}216-\mathrm{A}-5 \\
216-\mathrm{A}-10 \\
216-\mathrm{A}-4\end{array}$ & $\begin{array}{l}6(9) / 3 / 2 \\
5(9) / 1 \\
3(9) / 2\end{array}$ & $\begin{array}{l}\text { I All Times } \\
\text { I1/I2 } \\
\text { U1/U2 }\end{array}$ \\
\hline $\mathrm{C}$ tank farm \& vicinity & $\begin{array}{l}\text { UPR-200-E- } \\
81 \\
82 \\
86\end{array}$ & $\begin{array}{l}3 \\
4 / 4 \\
4 / 1\end{array}$ & $\begin{array}{l}\text { I3 } \\
\text { Tc1/Tc2 } \\
\text { Tc2/Tc3 }\end{array}$ \\
\hline $\mathrm{T}$ tank farm \& vicinity & $\begin{array}{l}241-\mathrm{T}-103 \\
241-\mathrm{T}-109 \\
241-\mathrm{T}-106 \\
241-\mathrm{T}-101\end{array}$ & $\begin{array}{l}1 / 2 / 3 \\
1 \\
1 \\
1\end{array}$ & $\begin{array}{l}\mathrm{I} 1 / \mathrm{I} 2 / \mathrm{Tc} 2 \\
\mathrm{Tc} 2 \\
\mathrm{I} 3 \\
\mathrm{I} 2\end{array}$ \\
\hline Solid Waste - 200-ZP-1 & 218-W-3AE & 3 & Tc3 \\
\hline U cribs & $216-U-12$ & 2 & U3 \\
\hline All others in $200-\mathrm{ZP}-1$ & $\begin{array}{l}\text { UPR-200-W- } \\
135 \text { and } \mathrm{W}-2\end{array}$ & 2 & Tc1 \\
\hline U ponds and ditches & 216-U-10 & 1 & U1 \\
\hline
\end{tabular}


The remedy evaluation framework is intended to enable refinement of initial SAC-based site evaluation (e.g., from Table 2) using a structured approach to improve the evaluation of impact to groundwater and feed directly into remedy selection efforts. The approach would help identify those sites for which natural attenuation alone is likely sufficient (e.g., where additional remedy development is not needed) and highlight sites for which natural attenuation will need to be augmented. For sites where natural attenuation must be augmented, the approach is also intended to facilitate determining how to target remedy development and implementation in terms of the location and extent of actions that are needed to meet groundwater protection goals (e.g., Table 2).

Application of the structured remedy evaluation approach presented in this report is recommended as part of preparing for future feasibility studies and the process for remedy selection mandated by the Comprehensive Environmental Response, Compensation, and Liability Act and the Resource Conservation and Recovery Act. Below, several examples of site conditions and associated data that illustrate some portion of the evaluation approach are presented.

Uranium contamination near the $B X$ Tank Farm (BX-102). There is a uranium contamination plume in the groundwater in the $\mathrm{B} / \mathrm{BX} / \mathrm{BY}$ Area (Figure 13). A recent series of reports, including Lindenmeier et al. (2008) and Serne et al. (2008, 2010), developed an update of the conceptual model for the BX-102 area based on recent data collection and identified key information about the uranium distribution in the vadose zone. The data in Figure 14 show that there is a significant inventory of uranium in the vadose zone. However, a large portion of the uranium inventory is in chemical forms that are functionally immobile. The amount of remaining mobile uranium in the vadose zone has been estimated, and the mobile inventory may be impacted by additional attenuation processes during transport. Figure 14 also illustrates that, currently, the majority of the inventory is in the vadose zone, with only a small amount within the groundwater. Thus, to date, uranium migration through the vadose zone has been slow and has resulted in some of the uranium mass becoming functionally immobile.

In this area, the Cold Creek Unit (CCU) low-permeability layer is structured such that a perched water zone is present and has intercepted movement of contaminated water through the vadose zone and thereby attenuated the flux of contaminated water through the vadose zone. As shown in Figure 1C, the perched water zone is a feature that is expected to control the continued future flux of contaminated water into the groundwater. The $\mathrm{CCU}$ feature at BX-102 acts as a limit for the future flux of mobile uranium. The characteristics of the perching layer control the water flux under current perched water conditions and relate to the water saturation conditions that would be expected once the perched water dissipates. Investigation of the properties of the perching zone could be used to estimate the water saturation conditions that would correspond to recharge-driven conditions.

Figure 15 illustrates the elevated water content at and above the CCU ( 220-230 ft bgs) and at other lower-permeability layers. Well 299-E33-45 is near BX-102 and was significantly impacted. The remedy evaluation framework can be used to evaluate attenuation associated with the perched water and other processes associated with predicting the contaminant flux to groundwater. Key remediation decisions supported through refining the estimate of flux at this controlling features are 1) determining the amount of water removal that will hasten return to recharge-driven conditions (at the BX-102 site, there is also a need to confirm and consider the impact of poorly sealed well bores) and 2) predicting the uranium concentrations in groundwater for recharge-driven conditions to assess whether additional vadose zone or groundwater actions are necessary. 


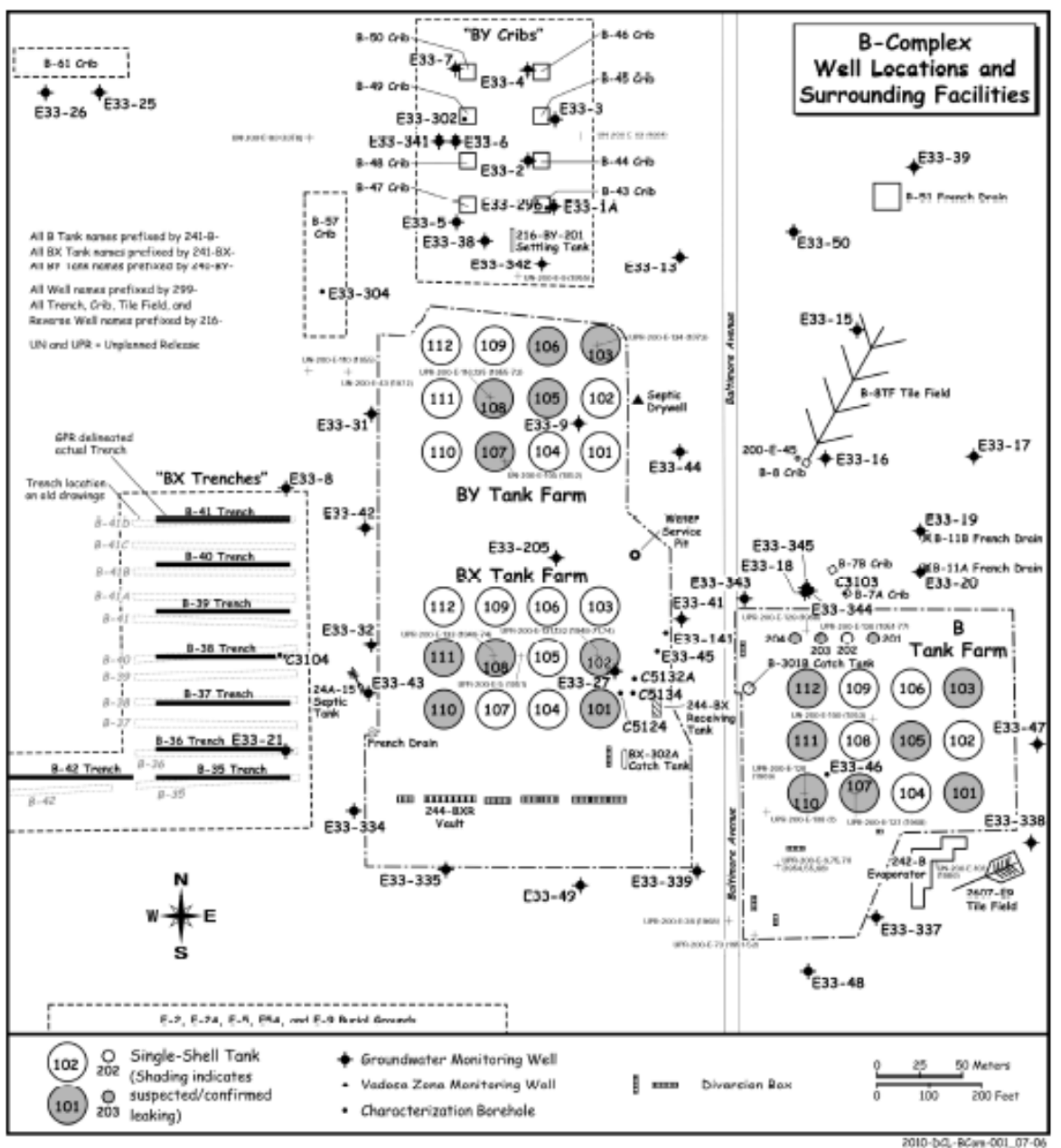

Figure 13. Site map indicating waste site and well locations for both BX and BY areas (Serne et al. 2010, Figure 1.1). 


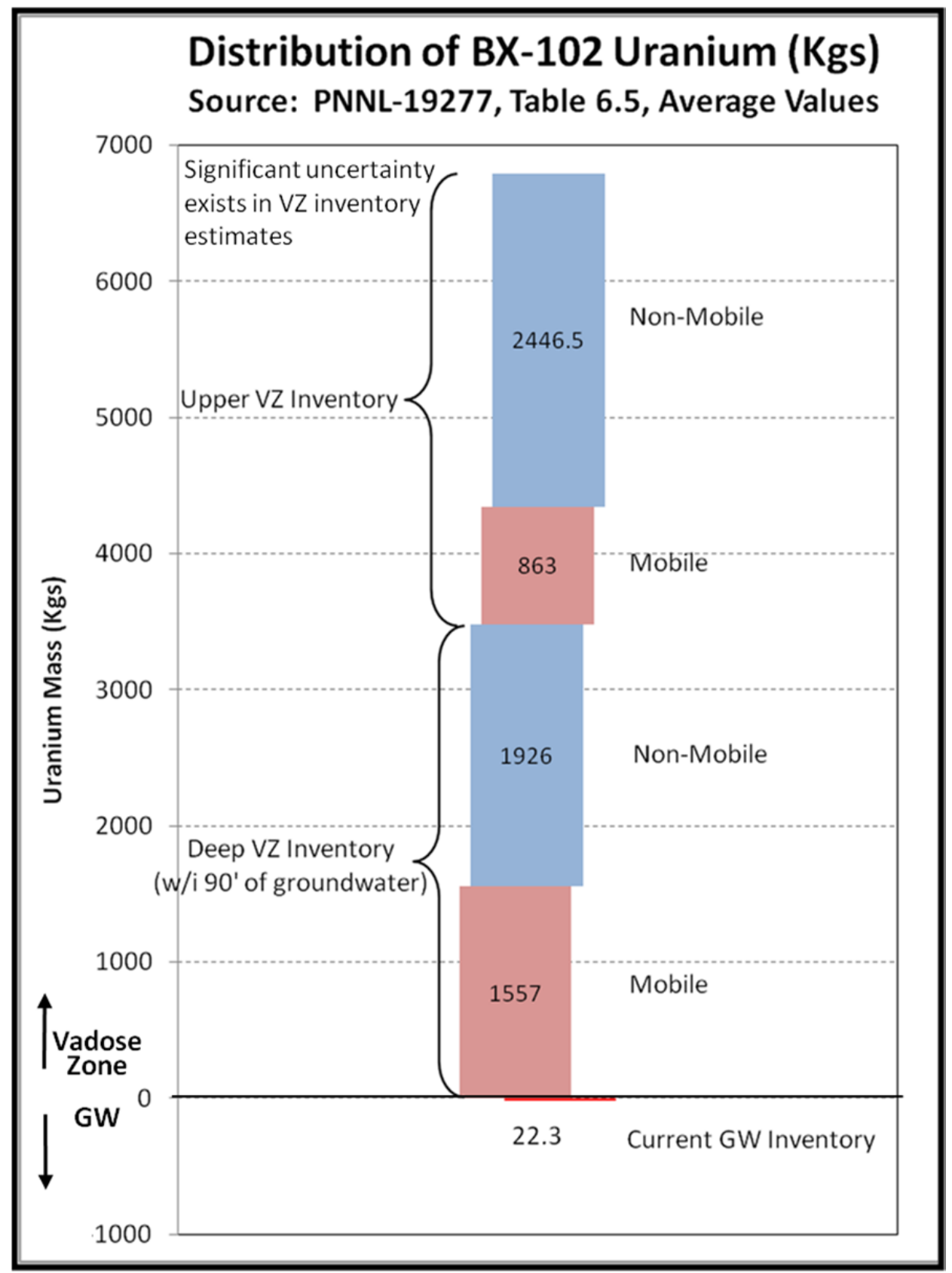

Figure 14. Depiction of the estimated uranium inventory in the vadose zone at the BX-102 site. Data from Serne et al. (2010). 
299-E33-45 (near Tank 241-BX-102)

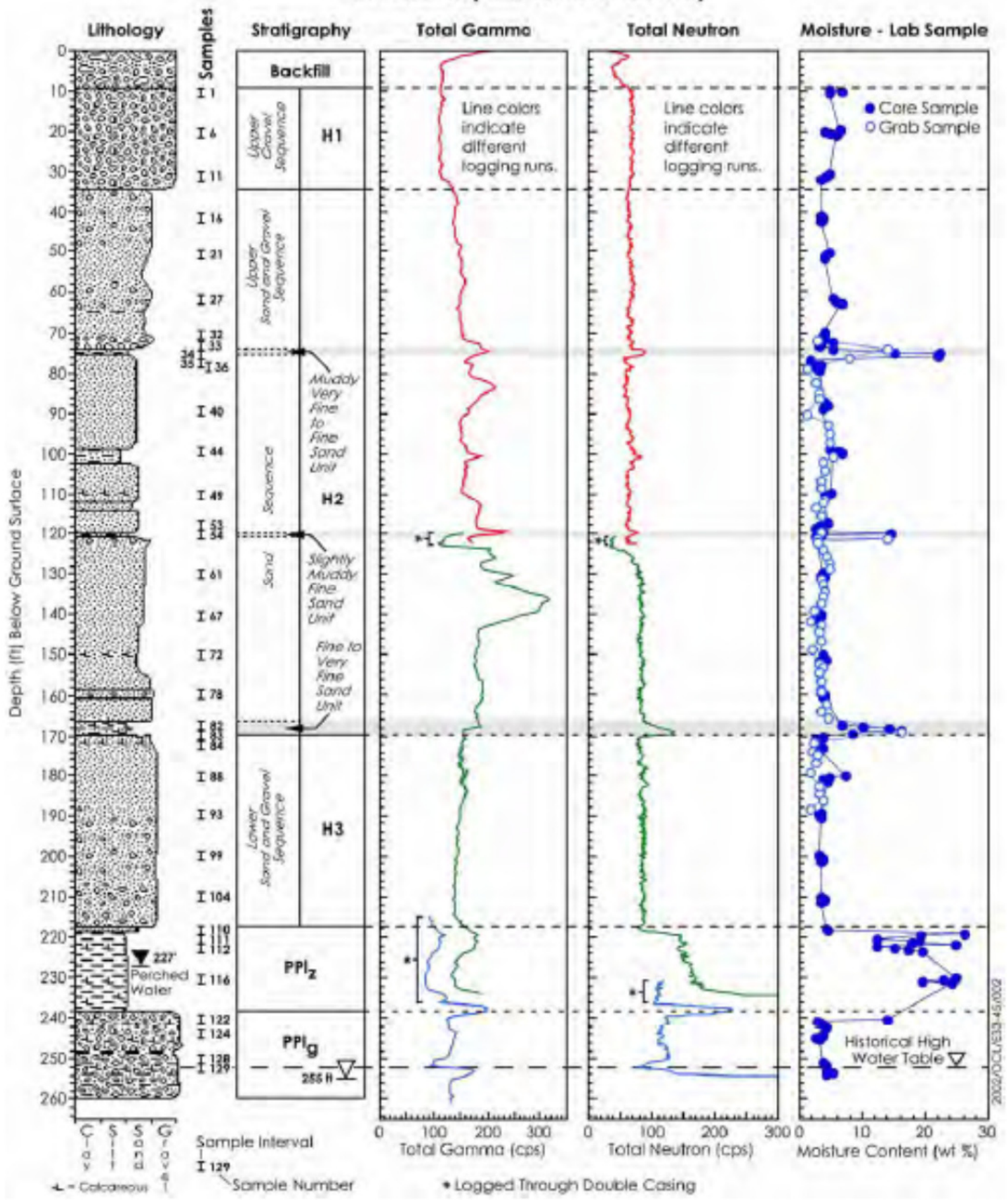

Figure 15. Lithology, stratigraphy, geophysics, and water content versus depth for well 299-E33-45 (Serne et al. 2008). 
In addition to illustrating the impact of low-permeability zones in interrupting vertical water migration through the vadose zone and producing perched water zones, other data collected as part of previous BX-102 conceptual model efforts highlight other components that may be important to consider in applying the remedy evaluation framework.

Figure 16 illustrates the pore-water concentration profiles for several contaminants in the BX-102 area well 299-E33-45. Note that the elevated concentrations are generally above the lower-permeability layers, which can be used to evaluate attenuation behavior.

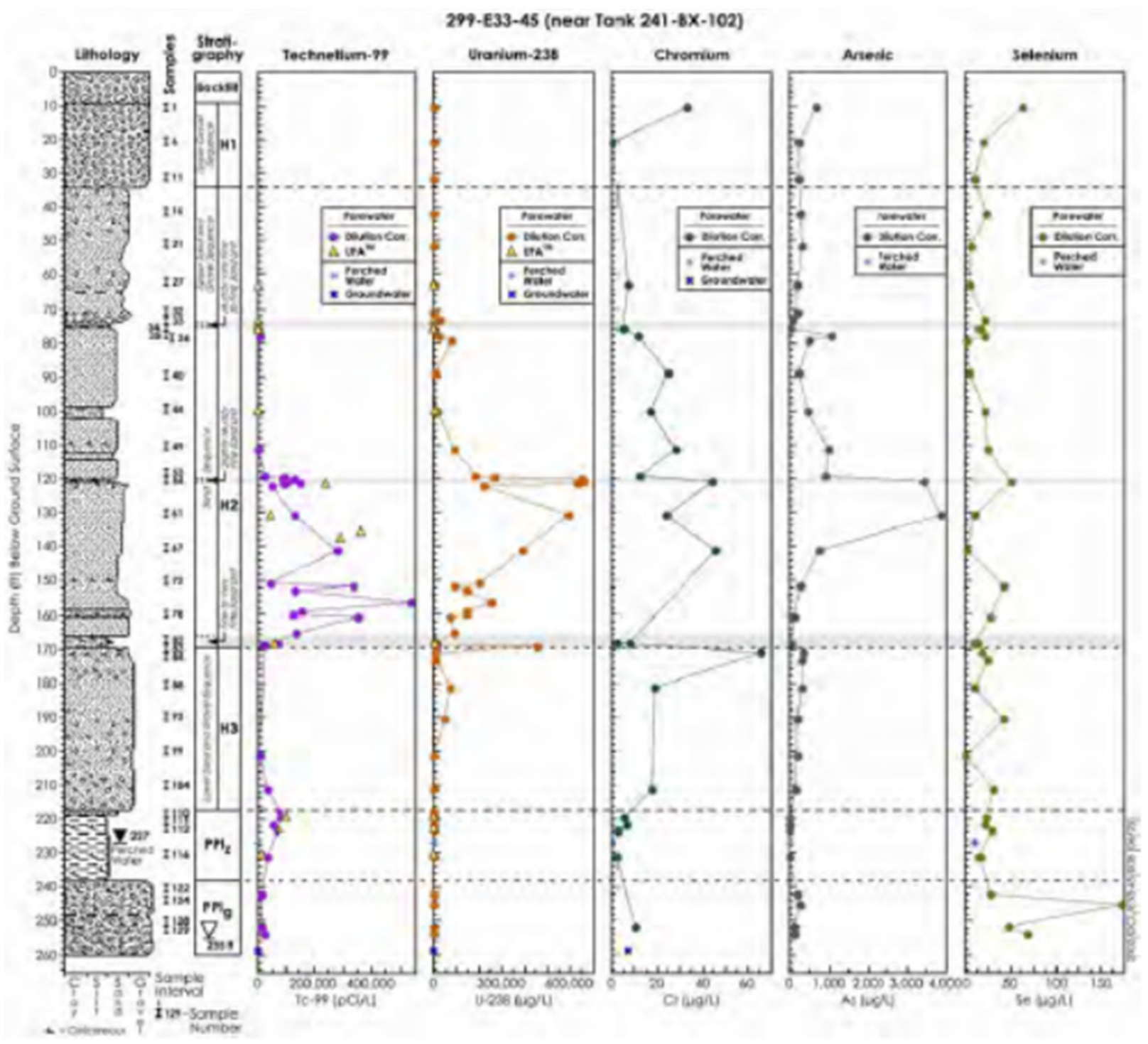

Figure 16. Lithology, stratigraphy, geophysics, and pore-water concentrations versus depth for well 299-E33-45 (Serne et al. 2008).

Water (A) and acid (B) extractable U concentrations with depth at BX-102 and at well 299-E33-45 are presented in Figure 17, illustrating that a significant amount of the uranium is immobile (acid extractable) and supports the observations in Figure 14. 

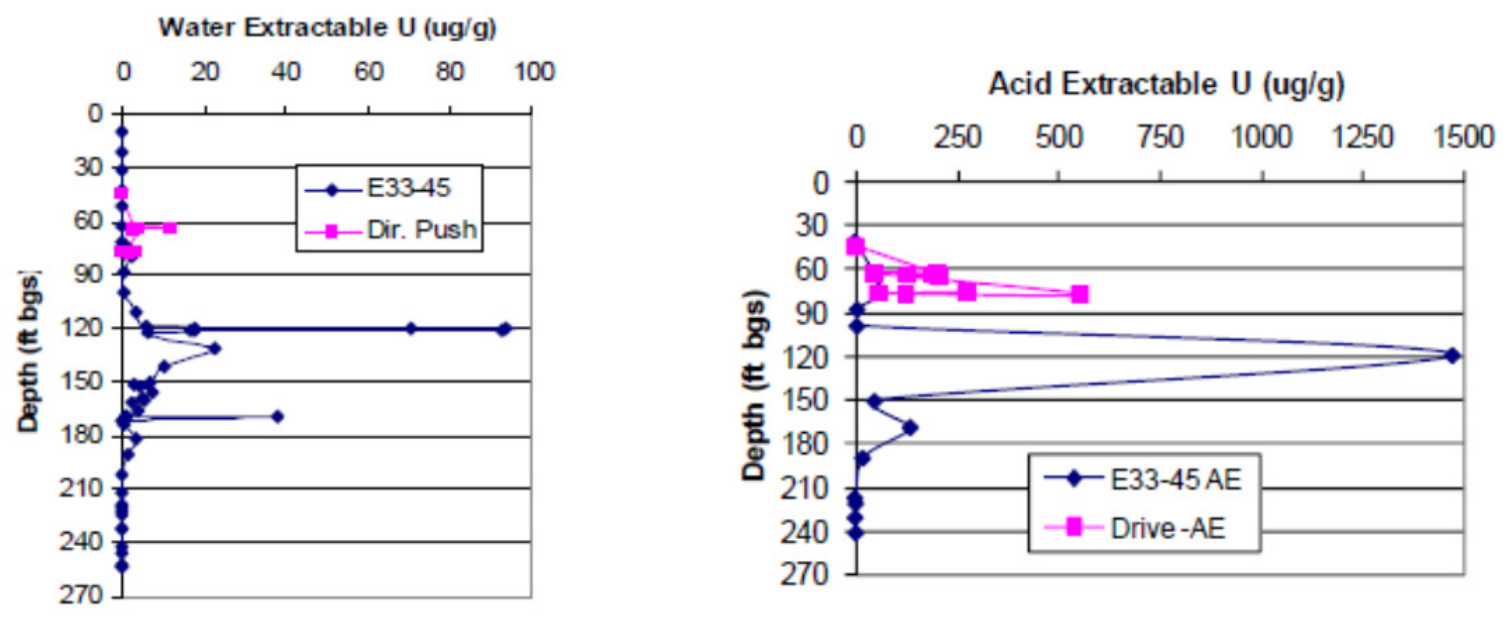

Figure 17. Water (A) and acid (B) extractable U concentrations with depth at BX-102 and at well 299-E33-45 (Serne et al. 2010).

Matric potential data were measured by filter paper technique and are presented below with depth along 299-E33-45 (Figure 18) and 299-E33-338 (Figure 19). Well 299-E33-45 is near BX-102 and was significantly impacted, and well 299-E33-338 is farther away from BX-102. Both locations have drastic changes near and below the CCU, which likely are due to the changes in permeability above and below the CCU that cause the perching and lateral spreading of water. Above the CCU at well 299-E33-45, the core matric potential is low for the entire profile, and the data are much lower than the line (representing unit-gradient, steady-state conditions). This suggests that vadose zone water flux is still downward and greater than expected for natural recharge conditions. At well 299-E33-338 above the CCU, matric potential is generally higher than at well 299-E33-45, which indicates a lateral hydraulic gradient and the potential for lateral spreading. This type of data supports the evaluation of the hydraulic gradient and the potential for vertical and lateral flow.

Technetium contamination beneath the SX Tank Farm. Similar to the BX-102 example, the CCU may be controlling flux through the vadose zone, causing high moisture conditions above and within the CCU (Figure 20 and Figure 21). As with the BX-102 example, moisture conditions in finer grained zones such as the CCU are expected to be relatively high under recharge-driven conditions. The Tc-99 data collected in boreholes surrounding the tank farm show that lateral spreading has occurred with Tc-99 observed up to $50 \mathrm{~m}$ from the tanks. The lateral spreading diminishes moisture conditions as an attenuation mechanism and an assessment of moisture conditions and porous media properties could be used to assess current fluxes in relation to the fluxes expected under long-term recharge-driven conditions.

The remedy evaluation framework may be used to evaluate attenuation associated with the high water content CCU and other processes associated with predicting the contaminant flux to groundwater. Key remediation decisions supported through this type of analysis could identify the extent of water removal that would hasten return to recharge-driven conditions (e.g., through pore-water extraction or desiccation). Prediction of Tc-99 concentrations under recharge-driven conditions could be used to assess the need for and extent of Tc-99 removal from the vadose zone (e.g., pore-water extraction), or to support consideration of other mitigation approaches (e.g., significant desiccation, surface infiltration barriers). 


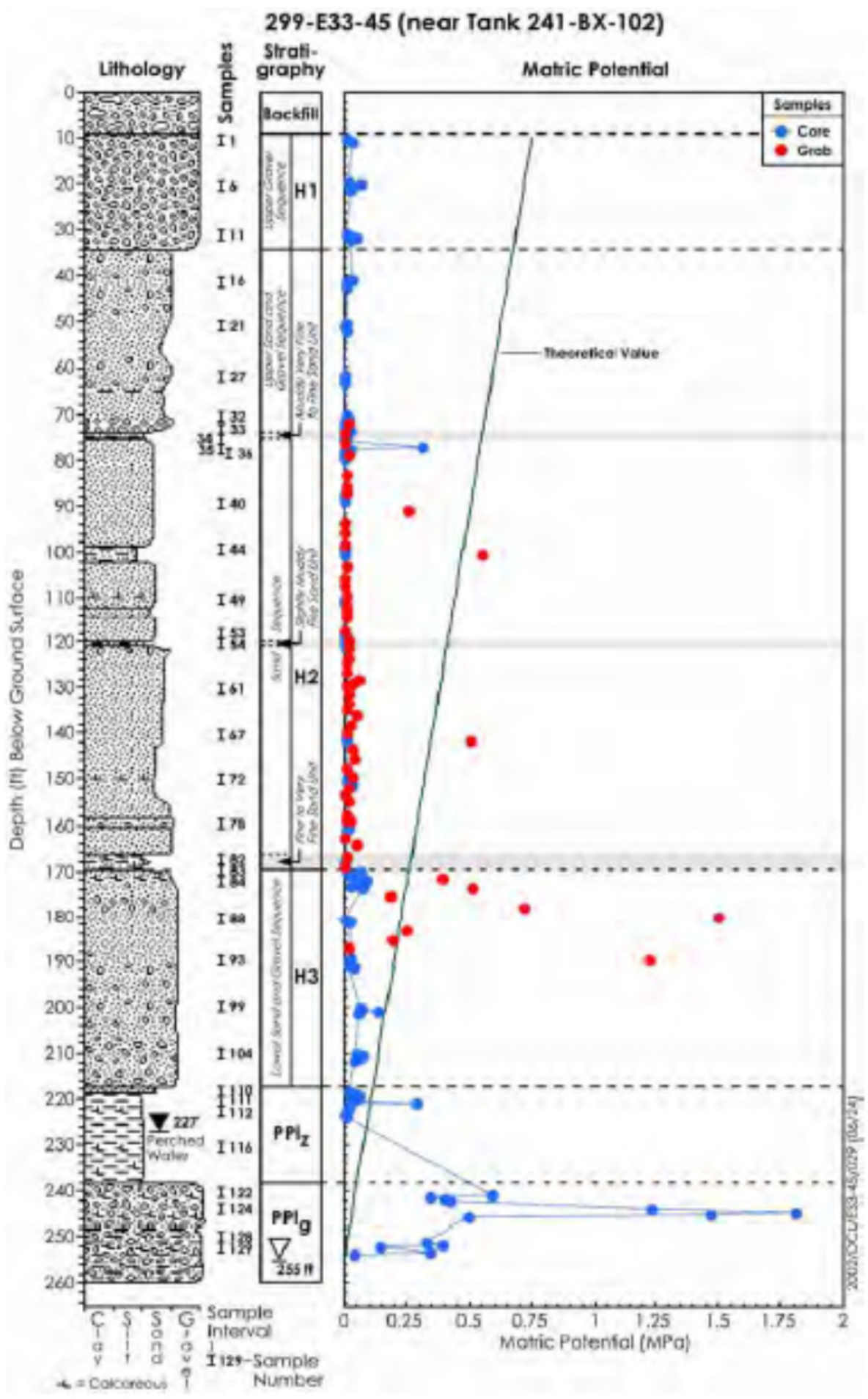

Figure 18. Matric potential by filter paper technique on core and grab samples with depth along well 299-E33-45 (Serne et al. 2008). 


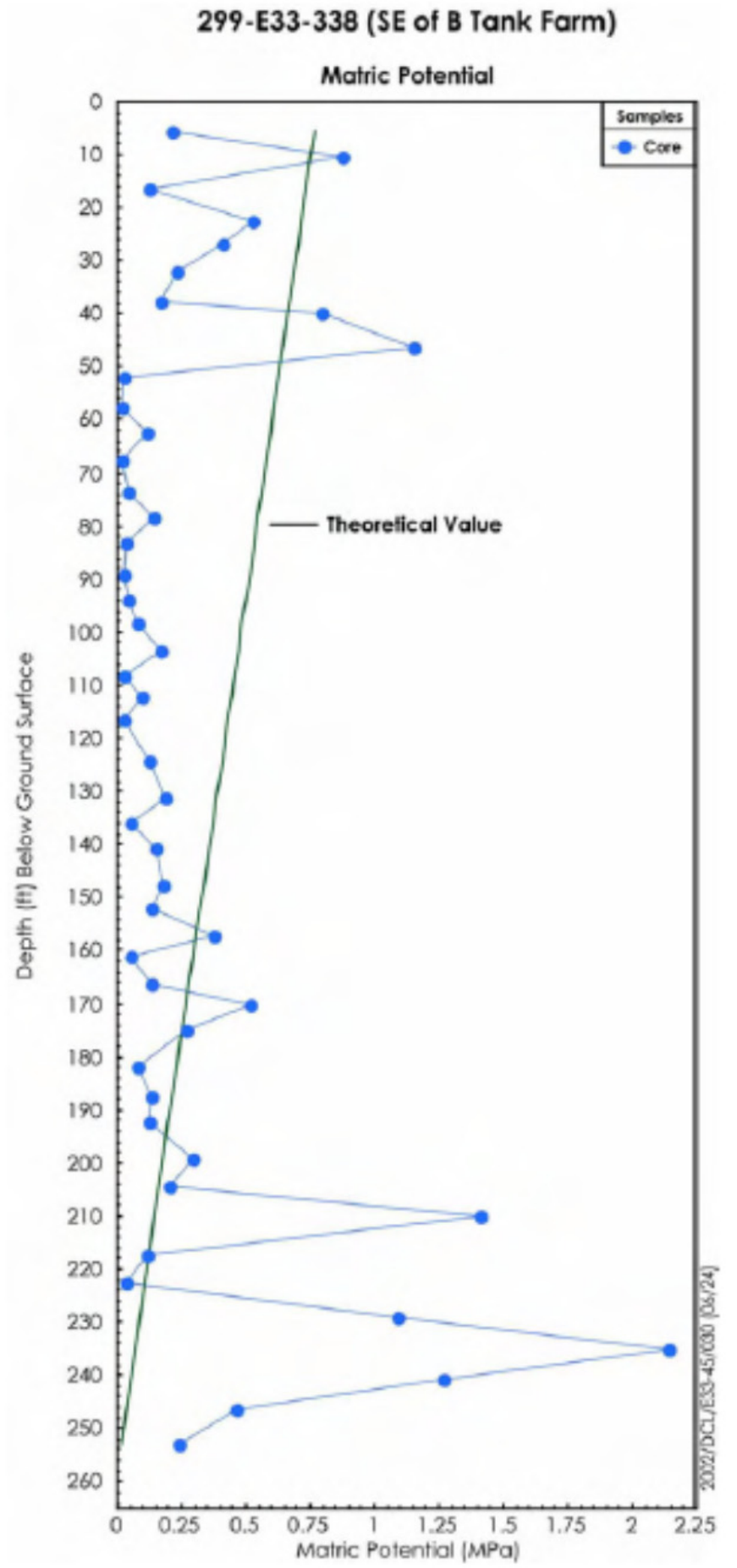

Figure 19. Matric potential by filter paper technique on core samples with depth along well 299-E33-338 (Serne et al. 2008; Lindenmeier et al. 2008). 
299-W23-19 (B8809)

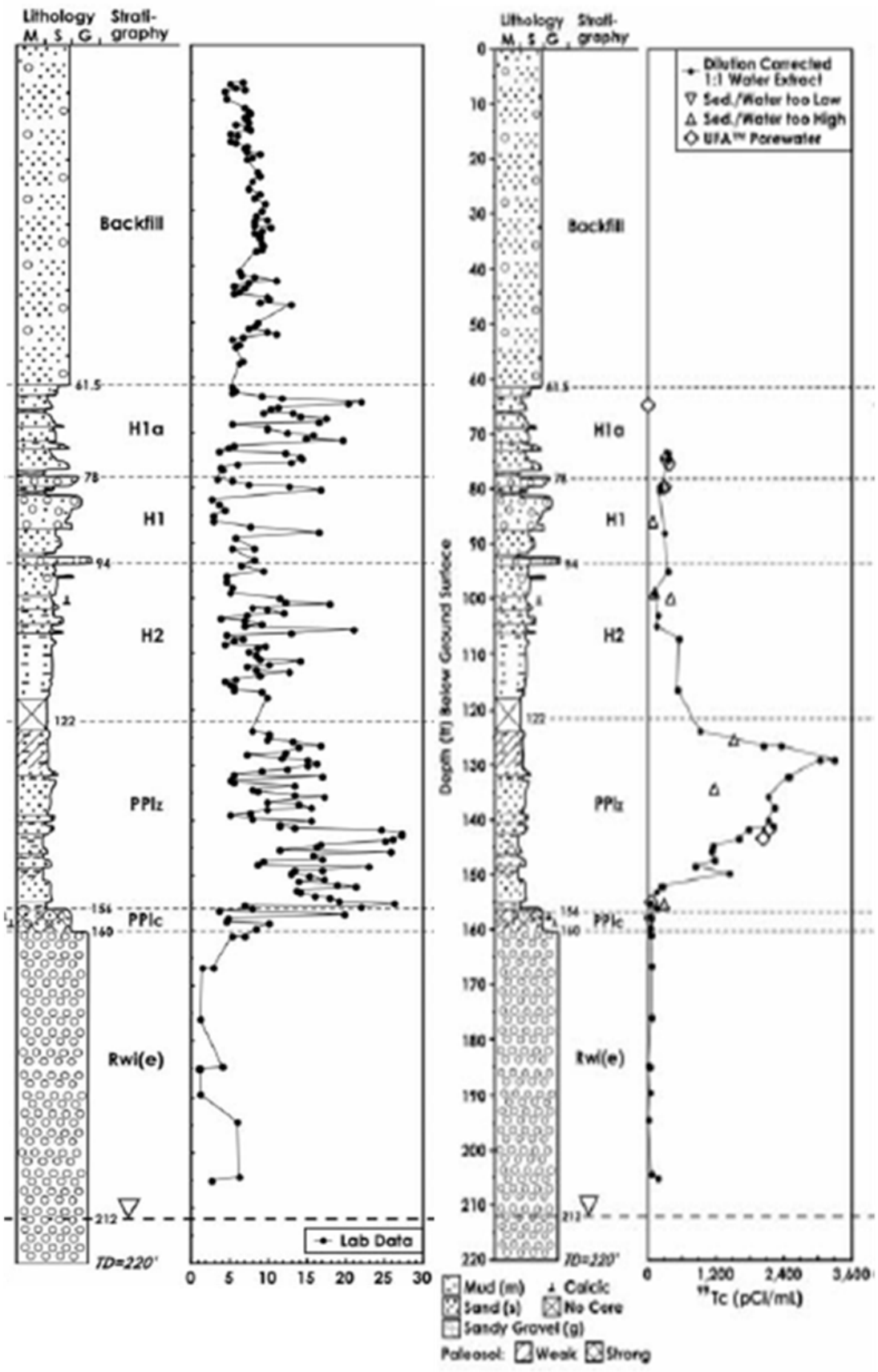

Figure 20. Lithology, stratigraphy, and moisture and Tc-99 profiles at the 299-W23-19 borehole at the edge of the SX tank area. 


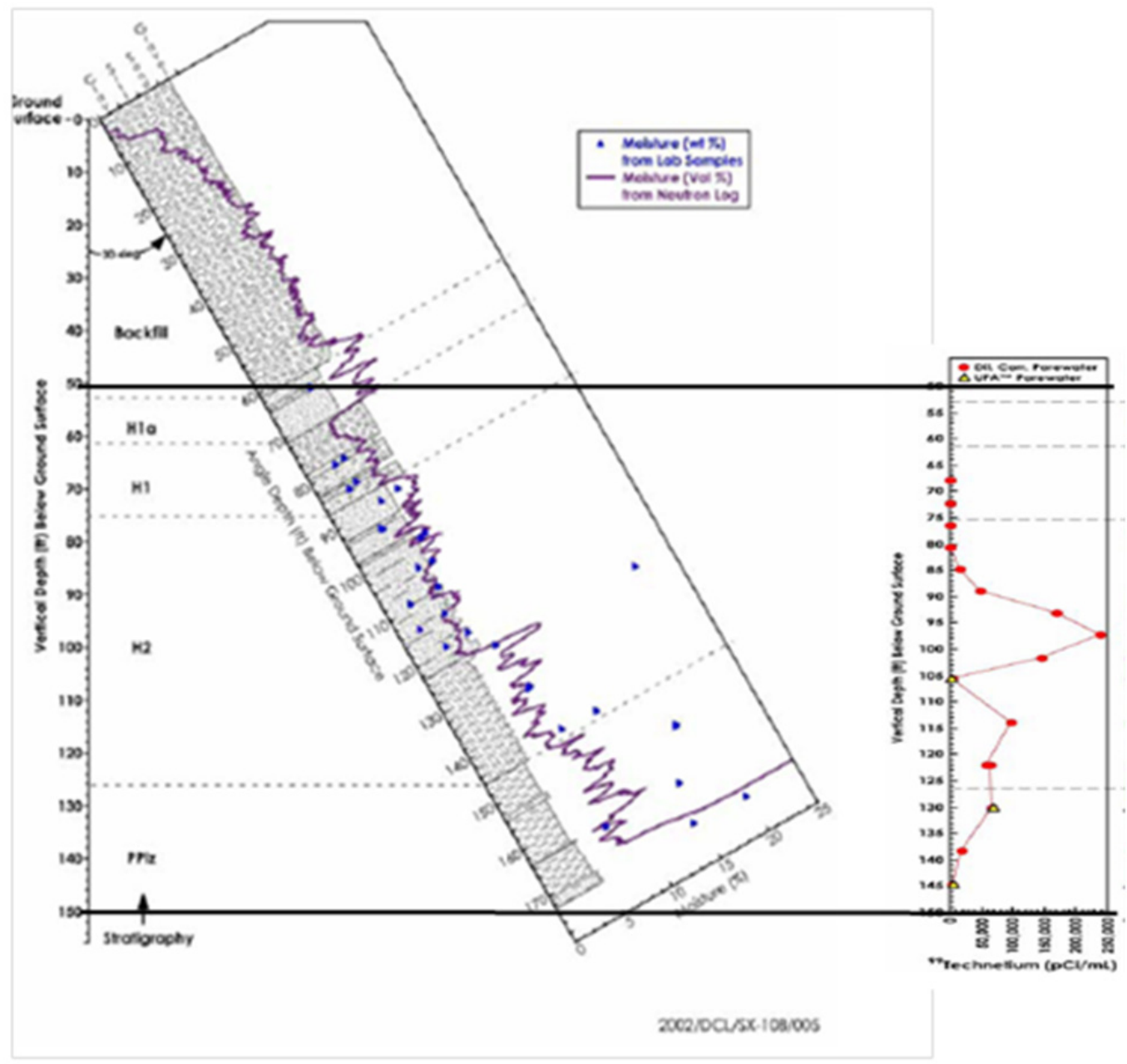

Figure 21. Moisture and Tc-99 profiles at the slant borehole beneath the SX-108 tank.

Technetium contamination at the BC Cribs and Trenches site. The CCU is not present in the vadose zone beneath the BC Cribs and Trenches site (Figure 22). Thus, similar to the BY Cribs example and unlike the BX-102 and SX tank farm examples, moisture and contaminant flux would be expected to behave as shown in either Figure 1A or Figure 1B. Data, including electrical resistance surveys (Figure 23; Serne et al. 2009), show that lateral spreading of the contamination and associated added water has occurred. Similar to the BY Cribs example, lateral spreading diminishes moisture conditions as an attenuation mechanism and an assessment of moisture conditions and porous media properties could be used to assess current fluxes in relation to the fluxes expected under long-term recharge-driven conditions (e.g., to assess whether future fluxes are expected to resemble Figure 1A or Figure 1B). The BC Cribs and Trenches site has been the focus of vadose zone investigations (e.g., Ward et al. 2004; Truex et al. $2011 \mathrm{~b}, 2012$ ). These efforts have included simulation of water and Tc-99 flux to groundwater (Ward et al. 2004). These simulations predict that most of the Tc-99 will reach the water table under rechargedriven conditions (Figure 24), but at fluxes that cause the groundwater Tc-99 concentration to exceed the DWS. Additional simulations and investigation of Tc-99 transport in unsaturated sediments (Jansik et al. in review) is ongoing to refine the understanding of potential future groundwater impact at the BC Cribs and Trenches site. 
The remedy evaluation framework evaluation can be applied to evaluate processes associated with predicting the contaminant flux to groundwater. A refined conceptual model and evaluation of groundwater impacts can support decisions regarding potential need for and extent of active remedies at this site.
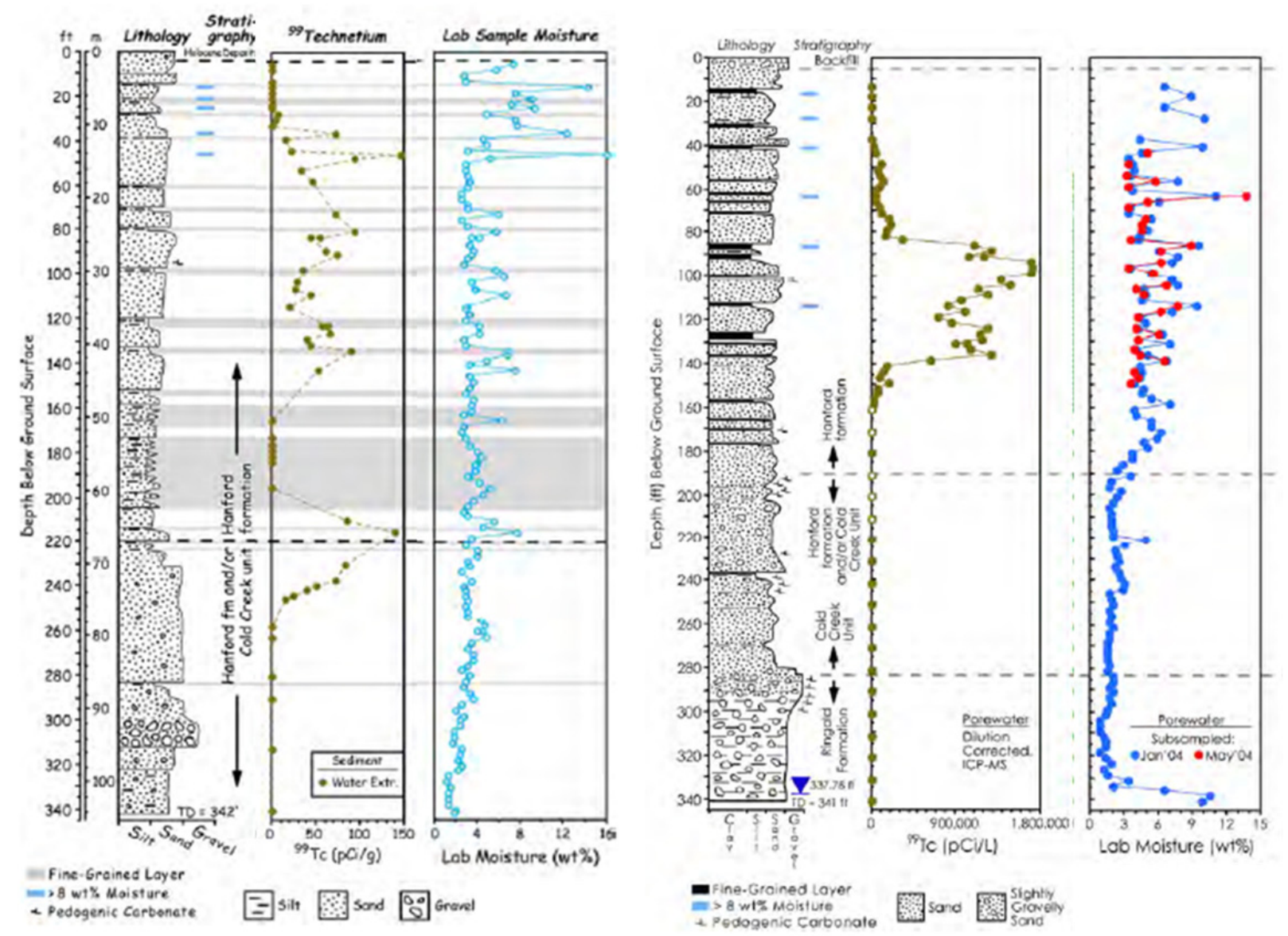

Figure 22. Laboratory-sample-derived Tc-99 and moisture profiles for borehole 5923 (left) located between four cribs and borehole 4191 (right) located adjacent to the B-26 trench at the BC Cribs and Trenches site (200-BC-1 Operable Unit). Note the 5923 data is at location offset from the cribs discharge such that information must be interpreted with respect to lateral moisture and contaminant flow and transport. Lenses of higher silt content materials are not necessarily laterally continuous at the $\mathrm{BC}$ Cribs and Trenches site. This figure is adapted from information reported in Serne et al. (2009).

Technetium contamination at the BY Cribs site. A conceptual model of the vadose zone was developed (Serne et al. 2010) identifying key information about the technetium (Tc-99) distribution in the vadose zone. The data in Figure 25 show that there is a significant inventory of Tc-99 in the vadose zone. Figure 25 also shows a significant inventory in the groundwater, suggesting that attenuation within the vadose zone has only partially mitigated Tc-99 contaminant flux. Tc-99 is not expected to undergo significant chemical alteration to immobile forms in oxic sediments, and thereby is expected to have less geochemical attenuation compared to a contaminant such as uranium. 


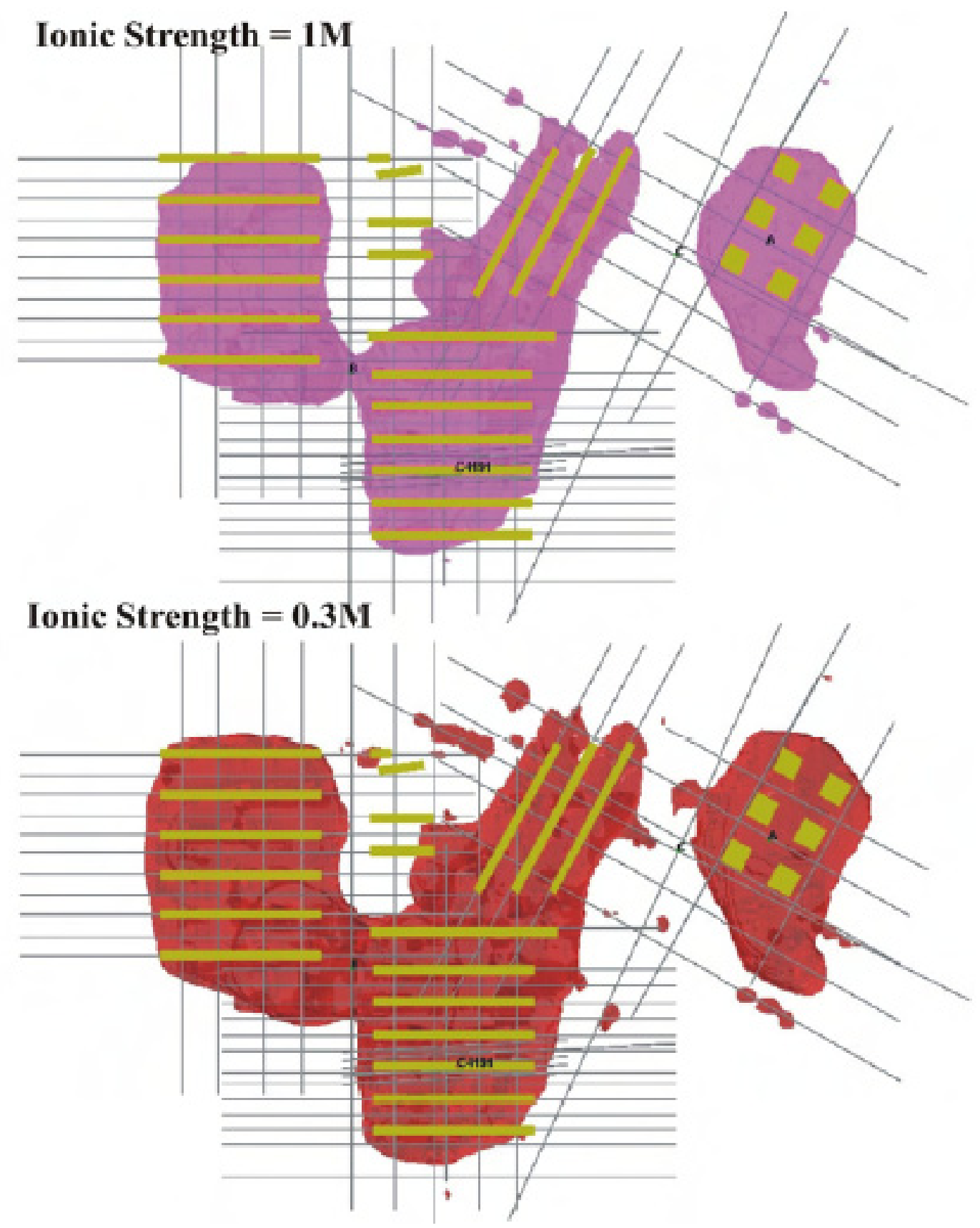

Figure 23. Estimated lateral distribution of ionic strength (used as a surrogate for contamination) in the vadose zone at the BC Cribs and Trenches site (200-BC-1 Operable Unit) (Serne et al. 2009). 

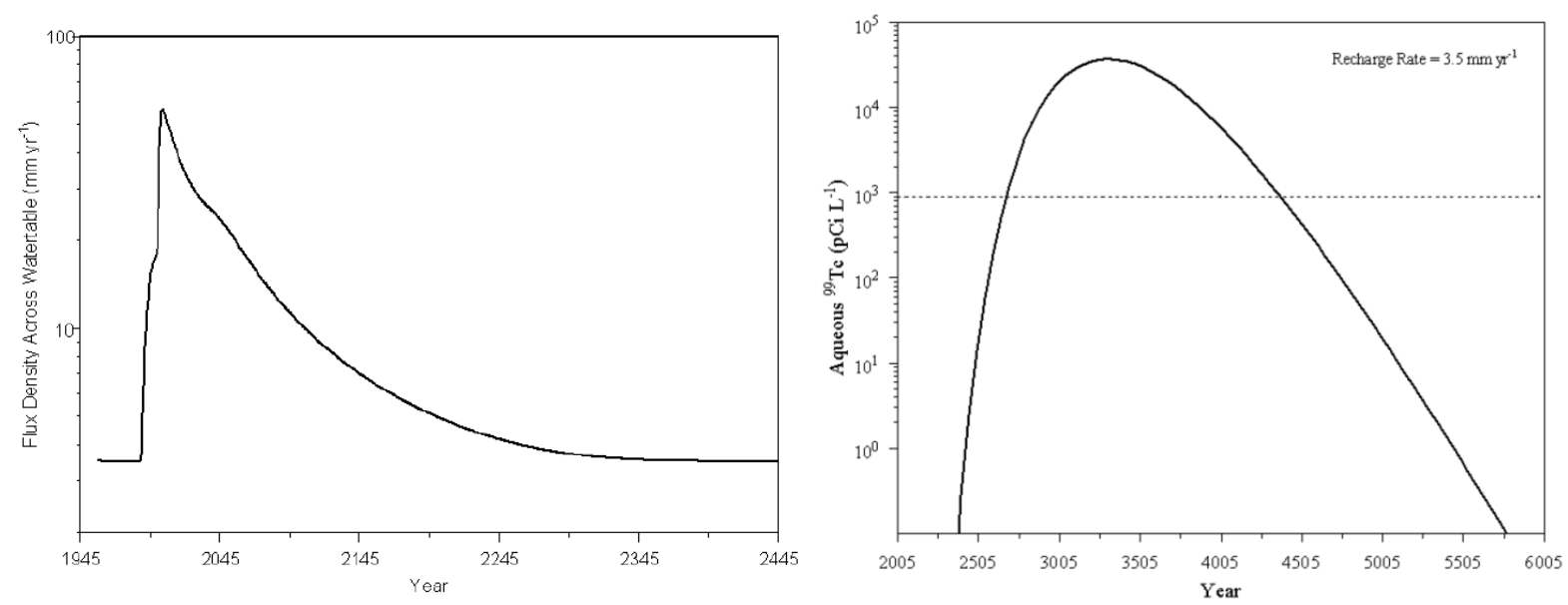

Figure 24. Simulated water flux across the water table (left) and predicted Tc-99 concentrations over time in the groundwater (right) beneath the trenches portion of the BC Cribs and Trenches site (Ward et al. 2004). The dashed line in the right plot is the Tc-99 drinking water standard. Note that the time scales are different on the two plots. Water flux has returned to recharge-driven conditions by about the year 2345 and the bulk of the Tc-99 enters the groundwater after that time.

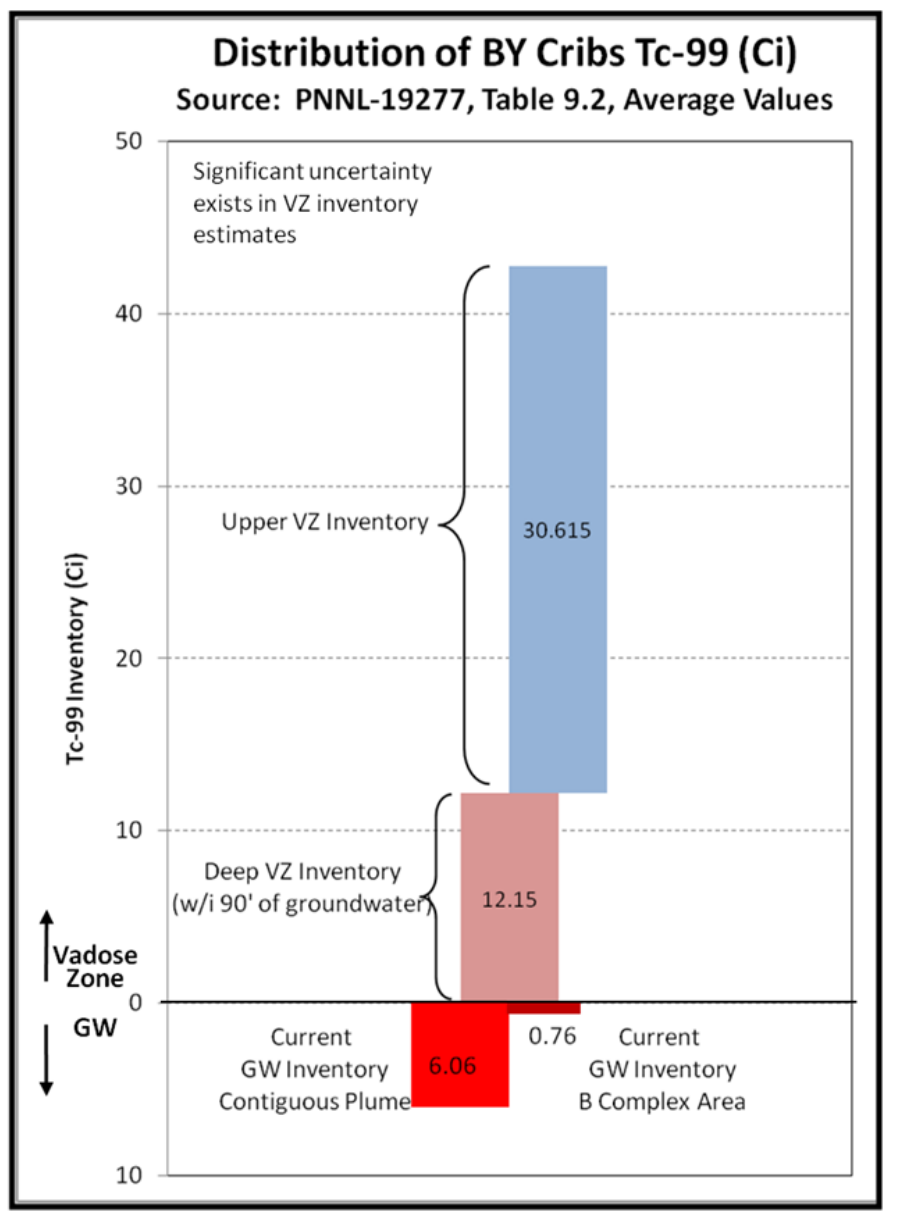

Figure 25. Depiction of the estimated technetium inventory in the vadose zone at BY Cribs using data from Serne et al. (2010). 
As with the BC Cribs area, the CCU is not present in the vadose zone beneath the BY Cribs (Figure 26). Thus, unlike the BX-102 and SX tank farm examples, moisture and contaminant flux would be expected to behave as shown in either Figure 1A or Figure 1B. Figure 26 for well 299-E33-342 at the BY Cribs suggests that there are no significant layers of lower permeability that mitigate vertical water flux.

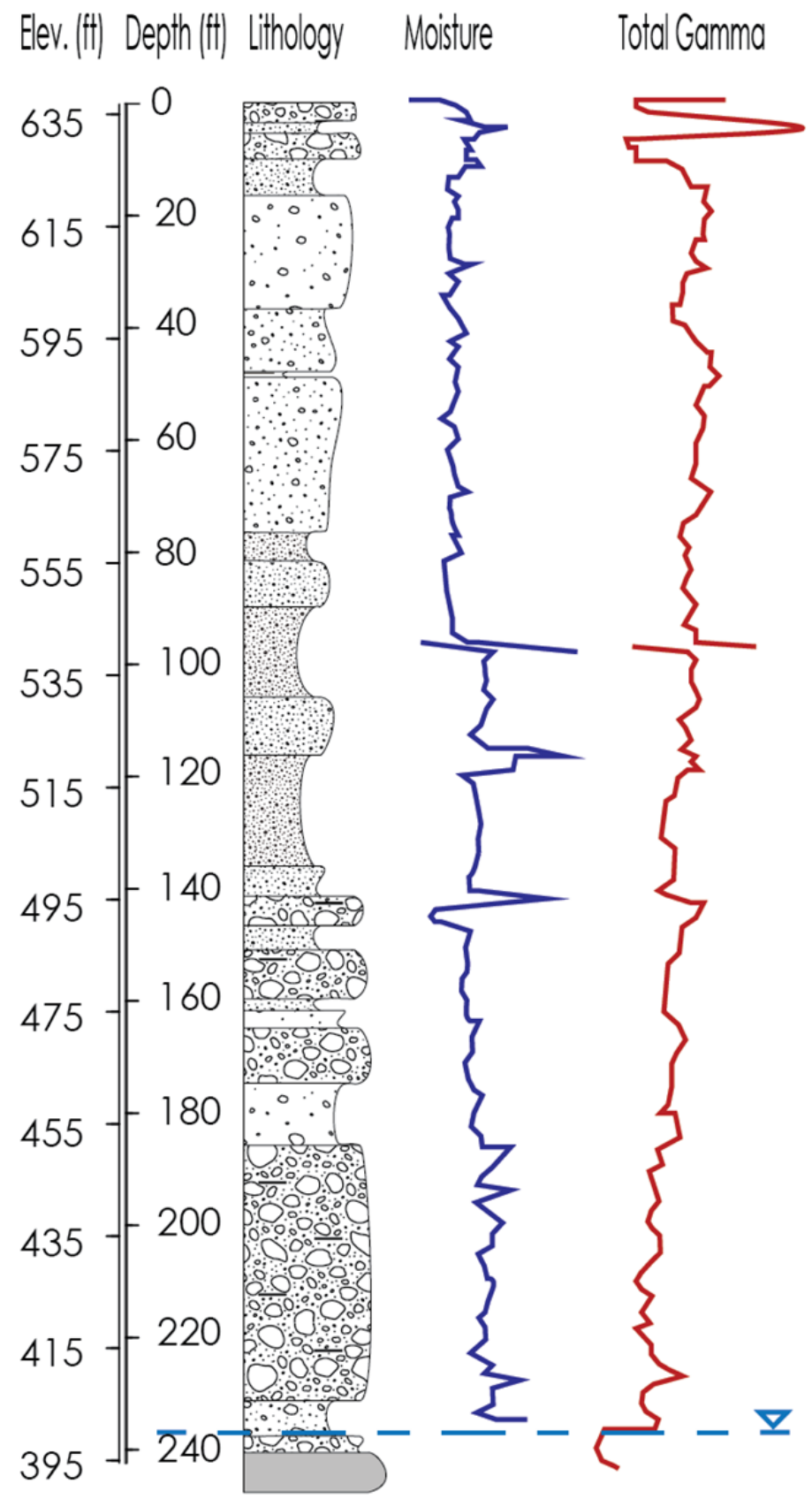

Figure 26. Lithology, total gamma, and water content versus depth for well 299-E33-342 in the $\mathrm{BY}$ area. Note that the low-permeability CCU is not present in this area. 
Data, including electrical resistance surveys (Figure 27 and Figure 28; Serne et al. 2010), show that lateral spreading of the contamination and associated added water has occurred. Similar to the SX Tank Farm example, although without the influence of the CCU as a controlling feature, lateral spreading diminishes moisture conditions as an attenuation mechanism, and an assessment of moisture conditions and porous media properties could be used to assess current fluxes in relation to the fluxes expected under long-term recharge-driven conditions (e.g., to assess whether future fluxes are expected to resemble Figure 1A or Figure 1B).

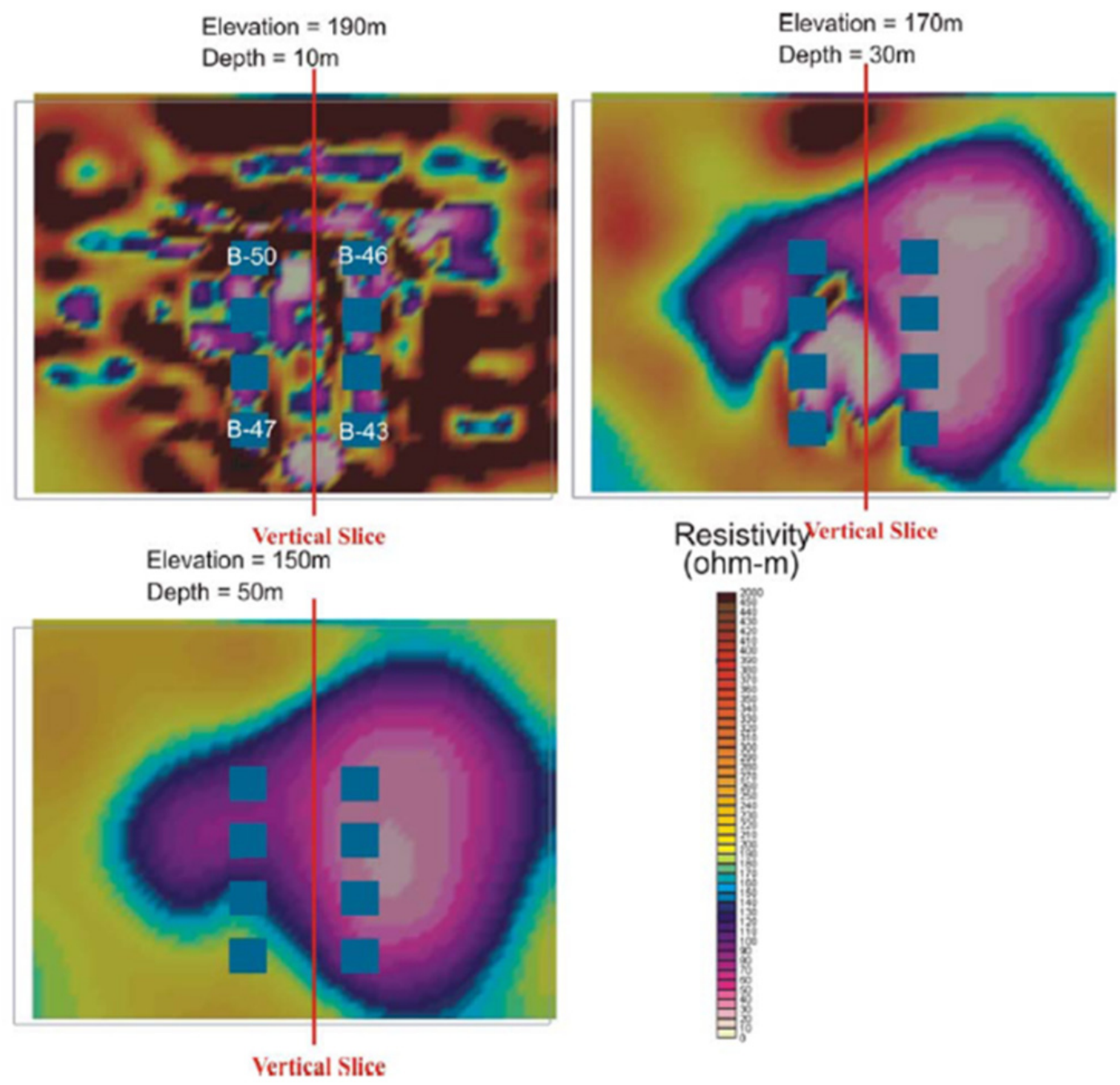

Figure 27. Electrical resistivity survey depicting lateral extent of low resistivity expected to correspond with the vadose zone contaminant distribution (Serne et al. 2010). 

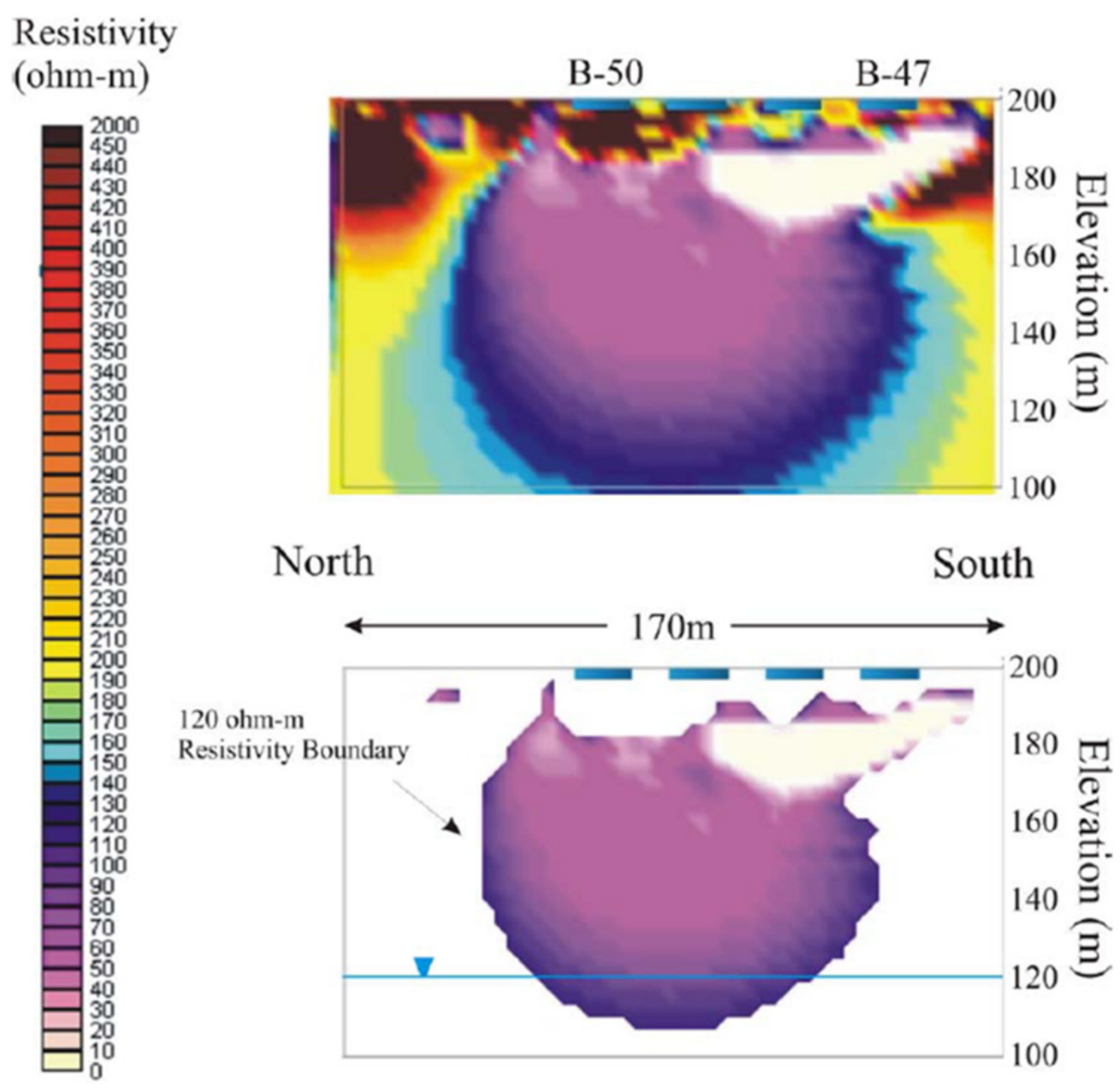

Figure 28. Electrical resistivity survey depicting a vertical slice of the subsurface where the lowresistivity region is expected to correspond with the vadose zone contaminant distribution (Serne et al. 2010).

Lateral water and contaminant spreading are also supported by the data presented in Figure 29. Figure 29 shows Tc-99 concentration profiles at the BY-49 Crib and well 299-E33-341. The waste was released at the location of the cribs, and contaminants present at the well indicate lateral spreading of the discharge waste.

Figure 29 also shows that Tc-99 has transported through the entire vadose zone and reached the groundwater at the BY area, contributing to a groundwater contamination plume. The current impact of this contaminant flux is depicted in Figure 30, showing the arrival of nitrate and Tc-99 from the vadose zone, mixing with the groundwater, and increasing the groundwater concentration. 


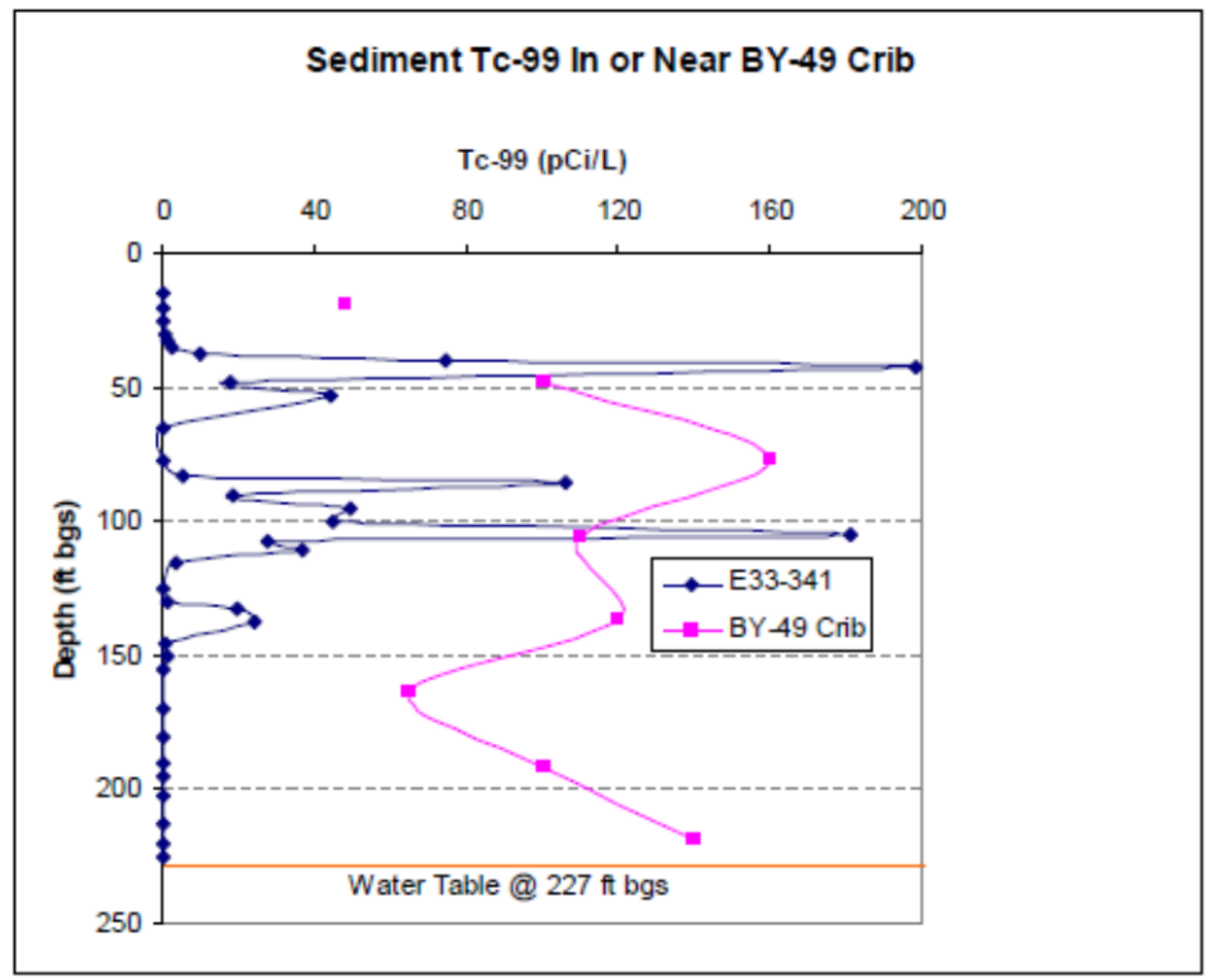

Figure 29. Tc-99 concentration profiles at the BY-49 Crib and well 299-E33-341 (Serne et al. 2010).

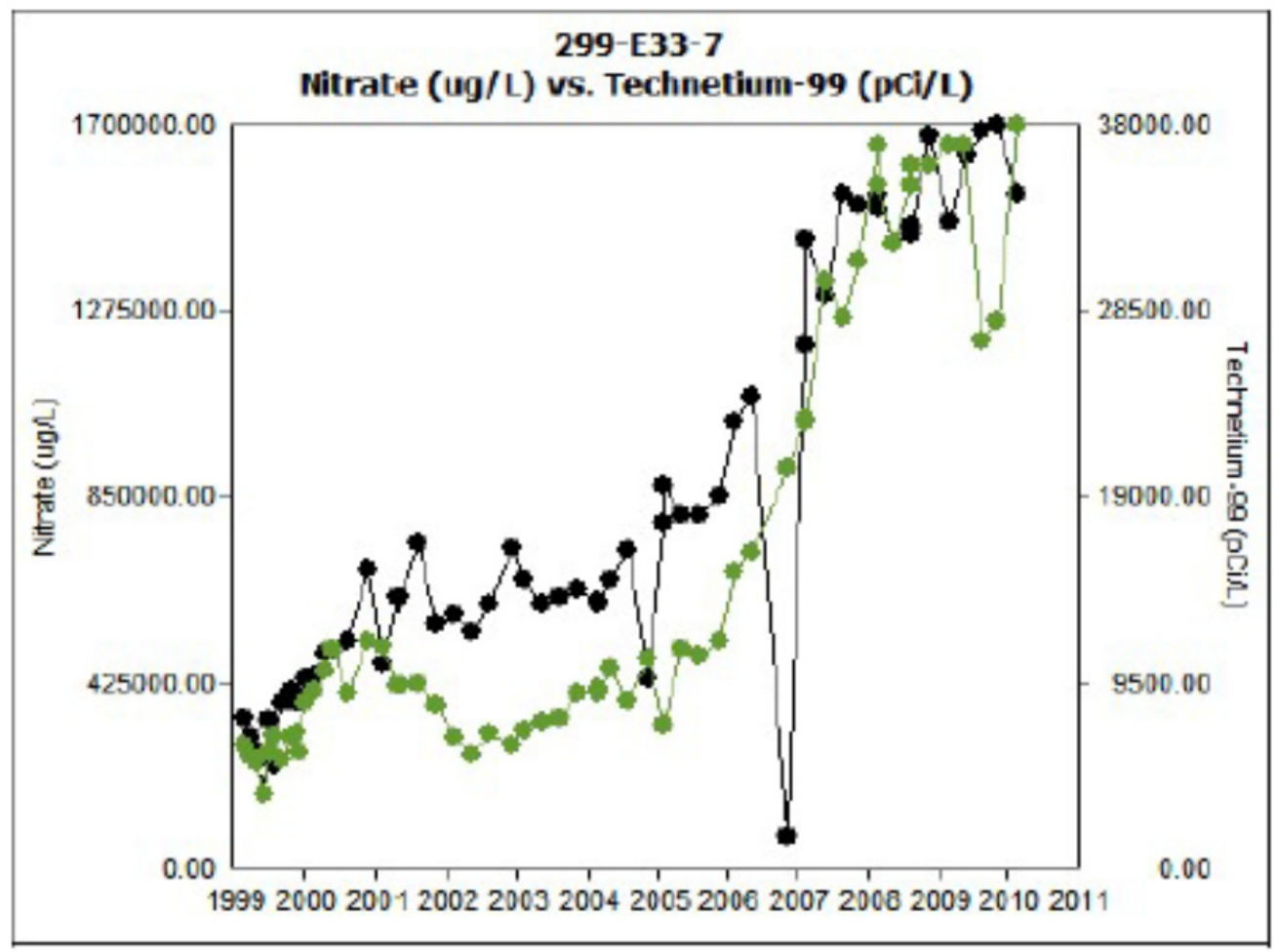

Figure 30. Groundwater concentrations over time of nitrate (black) and Tc-99 (green) at well 299-E33-7 in the BY area (Serne et al. 2010). 
At this BY area location, the remedy evaluation framework for the vadose zone can be applied to evaluate processes associated with predicting the contaminant flux to groundwater. Data collection and simulation or analyses conducted with the framework could be used along with interpretation of the existing groundwater plume to increase confidence in predictions of future impacts and selection of an appropriate remedy. Key remediation decisions supported through this type of analysis could identify the extent of water removal that would hasten return to recharge-driven conditions (e.g., through pore-water extraction or desiccation). In addition, prediction of Tc-99 concentrations under recharge-driven conditions could be used to assess the need for and extent of Tc-99 removal from the vadose zone (e.g., pore-water extraction) or to support consideration of other mitigation approaches (e.g., significant desiccation, surface infiltration barriers).

As illustrated in part by the case studies presented above, the type of data collected in previous and ongoing characterization and conceptual model efforts can be used to support key components of the remedy evaluation framework described in this report.

\subsection{Characterization and Monitoring Approaches Supporting Remedy Implementation}

A systems-based approach to characterization and monitoring, structured around refinement of a conceptual site model, is recommended to support the remedy evaluation framework described in this report and subsequent remedy implementation. Initial conceptual models should be refined through the framework presented in this document (illustrated in Figure 6) to support contaminant fate and transport modeling, baseline risk assessment, and remedy evaluation and selection. The framework and integration of characterization and monitoring are intended to be consistent with the approach for monitoring in the recently published Scientific Opportunities for Monitoring at Environmental Remediation Sites (SOMERS): Integrated Systems-Based Approaches to Monitoring (Bunn et al. 2012).

The rate of contaminant movement through the vadose zone (the mass flux and/or discharge) is a primary parameter used to assess potential impacts on groundwater. Direct measurement of contaminant flux in the vadose zone is rarely possible. However, this overall objective can be appropriately addressed using a lines-of-evidence approach in which the data collected are used to quantify the key factors related to contaminant migration and enable interpretation with respect to contaminant flux to the groundwater. Although each site is unique, a general conceptual model can be used to describe key characterization and monitoring targets (Figure 5 and Figure 31).

A primary goal of monitoring for non-volatile contaminants is to provide information useful to understanding and quantifying the contaminant distribution in the vadose zone and transport to groundwater. Characterization and monitoring data integrated into a descriptive conceptual model to identify the site-specific controlling features and processes are an important part of an overall strategy for predicting future states. Predictive (numerical) analyses are also part of that strategy, as are the iterative nature of monitoring and modeling. The results of predictive analyses and the refinement of these analyses with monitoring data can be important to the interpretation of monitoring data, in terms of verifying appropriate remedy performance. For most sites, waste disposal (time "a" on Figure 31) has already occurred, but important information may be available to describe site conditions during the time of active disposal (i.e., top curve). If these data are available, they can be related to predictions of, or 
monitoring approaches for, describing the contaminant flux to the groundwater (i.e., bottom curves). Key components of vadose-zone monitoring based on the generic transport framework (Figure 31) are discussed below and include the categories of 1) water flux, 2) contaminant flux and its relationship with water flux, 3) remediation processes, and 4) volumetric moisture and contaminant measurements.

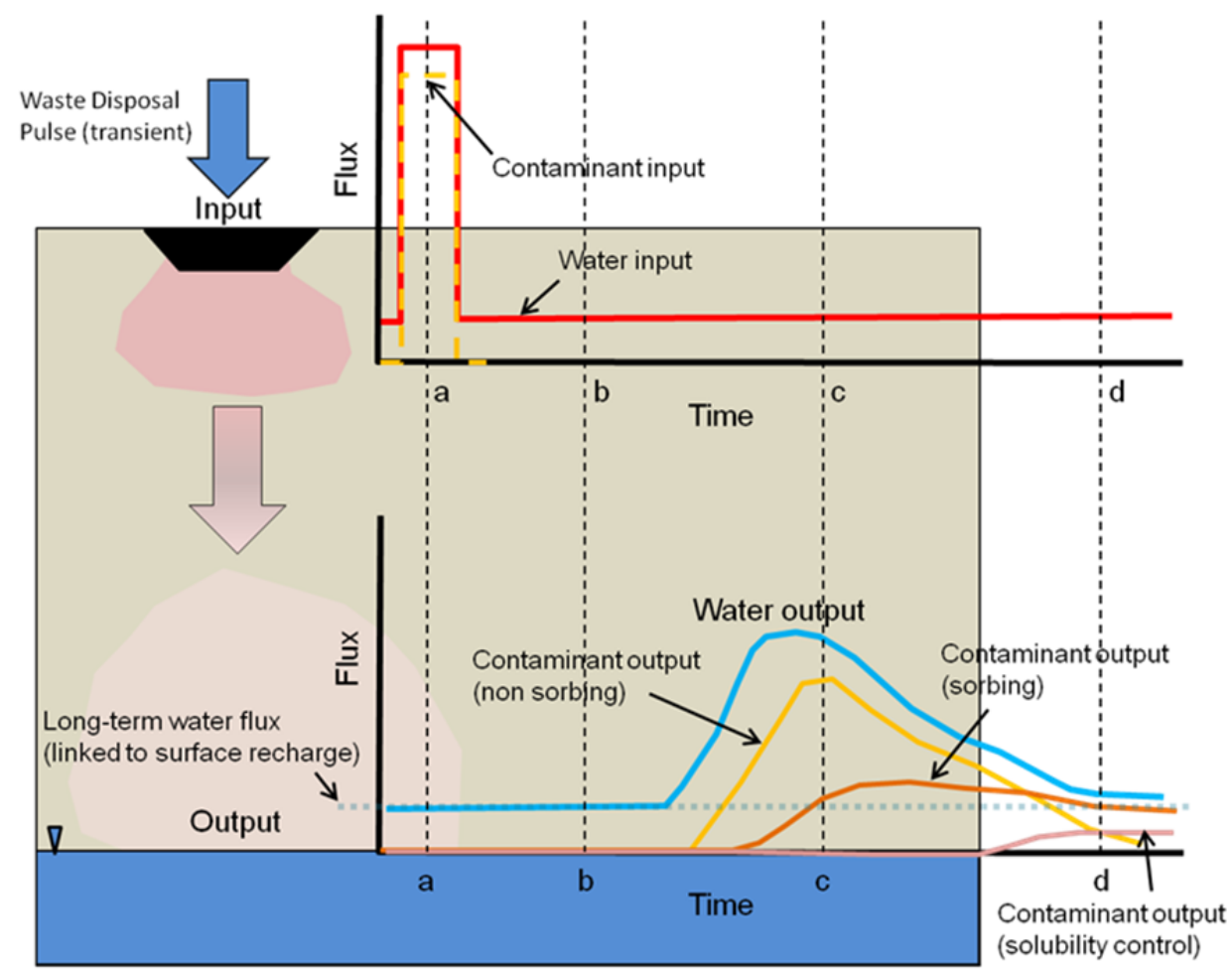

Figure 31. Generic description of the vadose zone as a transport path from the surface to groundwater for non-volatile contaminants.

1. Water Flux. The contaminant flux to groundwater is directly related to the water flux through the vadose zone. Figure 31 shows the generic progression of water flux over time. One target for monitoring (and predictive analysis) is determining the shape of the water output (from the vadose zone to the groundwater) flux curve over time and whether the site is currently at generic time "b," "c," or "d." At generic time "b," the water discharged with the waste disposal has not yet reached groundwater. At generic time "c," water flux at the water table is increased compared to pre-disposal conditions because the discharged water has reached the water table. At generic time "d," the peak of discharged water has passed into the groundwater and the water flux is at, or diminishing to a rate consistent with, long-term surface recharge conditions. Knowledge of whether the site is currently at generic time "b," "c," or "d" should be a goal of characterization, predictive analysis, or monitoring the water phase movement for those sites already impacted by anthropogenic activities. This type of knowledge may be useful in supporting streamlined monitoring approaches, especially for long-term monitoring if the dynamics of the contaminant flux are expected to be minor. The actual timescale and magnitude of the water flux changes are controlled by the discharge conditions, vadose zone properties, and their distribution (especially for controlling features such as interfaces between porous media zones with different properties) and the net surface recharge conditions. Thus, monitoring approaches that target these elements and focus on identifying changes over time are potential 
components of a vadose zone monitoring program. For most sites, especially progressing from time "c" to time "d," the recharge rate will be a primary indicator of water flux and no or only limited monitoring of other controlling features may be needed to define the water flux.

2. Relationship Between Contaminant and Water Fluxes. Contaminant flux and its relationship with water flux (and therefore, also the relationship between the contaminant and water distribution) is controlled by contaminant interactions with sediment surfaces (e.g., adsorption or precipitation/ dissolution processes) or by microscale transport phenomena such as intra-particle diffusion. Thus, lines-of-evidence vadose-zone monitoring may include monitoring for contaminant distribution (or surrogates such as ionic strength), factors that control the interactions (e.g., master variables for porewater chemistry), and/or indications of changes in interactions (e.g., sediment surface properties, ionic strength). The conceptual site model should indicate the type of contaminant interaction expected under either natural attenuation or remedy conditions. Thus, the conceptual site model would 1) identify the expected contaminant behavior relative to moisture movement (e.g., as depicted on Figure 31) and 2) potentially quantify expected fluxes for each contaminant (e.g., through geochemical, geological, and predictive analyses). Direct measurement of contaminant flux in the vadose zone is rarely possible. In cases where continued vadose-zone monitoring may be needed to verify the expected contaminant flux, use of periodic or time-lapse geophysical measurements may be a viable option. Periodic assessment of contaminant conditions at controlling features may also be possible. In some cases, measurement of groundwater contaminant concentrations may be a viable part of a monitoring strategy. Continued efforts to improve contaminant monitoring in the vadose zone, for instance as described by Bunn et al. (2012), may provide improved methods in the future. Because of the difficulty in direct measurement of contaminant flux, developing a technically defensible conceptual model and associated fate and transport evaluation is of critical for the vadose zone and is the basis of the structured approach for the framework presented in this report.

3. Volumetric Moisture and Contaminant Measurements. Volumetric measurements or measures of the vertical distribution of moisture and contaminant distribution and changes in these distributions can be interpreted in terms of current or future contaminant fluxes to groundwater. This type of approach provides information about the mass distribution of water and/or contaminants in the vadose zone. In some cases, monitoring the water flux can be simplified, especially for long-term monitoring. For instance, if water flux conditions for the site are at or near the long-term water flux (i.e., approximately equal to surface recharge rate), monitoring for water flux to groundwater reduces to monitoring of net surface recharge.

4. Remediation Processes. Some remedies impart specific changes to the vadose zone or contaminants that can be directly or indirectly (e.g., indicators or surrogates) monitored over time and related to contaminant flux to groundwater. Approaches may include monitoring of remedy impacts to vadose zone properties (e.g., permeability, sediment surface properties, pore-water chemistry), moisture conditions/distribution, contaminant properties (e.g., redox state, precipitates), or the remedy amendments (e.g., addition or removal of oxygen, addition or transformation of organic carbon, concentration of oxidant or reductant). All remedies target one or more processes that decrease water and/or contaminant flux, which may be monitored to evaluate remediation progress, as noted above.

The vadose-zone monitoring components discussed above have the potential to provide data that can be interpreted in a lines-of-evidence approach. These components have been discussed in the context of an overall objective to understand and quantify the contaminant flux to groundwater and associated vadose zone remedy performance. For each site, this goal can be applied at different levels of rigor 
appropriate to the overall remediation approach and the relative importance of the vadose zone contamination. For instance, some sites may require only a prediction of contaminant flux from the vadose zone to the groundwater and minimal confirmation of site conditions used to support this prediction. At other sites, where the vadose zone is more closely tied to a groundwater remedy, more detailed monitoring and quantification of contaminant flux may be needed. Thus, the monitoring design and program will also be related to decisions that need to be supported with the monitoring data. Table 2 also illustrates this type of prioritization across several sites that can inform both monitoring and remedy decisions. In any case, the components outlined in this section offer candidates for categories of vadosezone monitoring design that can provide data and information to interpret the impact of vadose zone contamination on groundwater quality within the framework of a conceptual site model and associated predictive analyses.

The framework presented here focuses on contaminant movement through the vadose zone. At sites with emplaced waste forms or previously buried waste, contaminants may not have been released to the subsurface but may be released over time. The same framework - viewing the vadose zone as a pathway to groundwater and the release as a transient event - applies in these cases. However, additional elements may be important to detect the releases. Each category of vadose zone monitoring discussed above may be applicable, although applied to locations and for targets relevant to the potential waste source and site conditions.

\subsection{References}

Brusseau ML, RE Jessup, and PSC Rao. 1989. "Modeling the Transport of Solutes Influenced by Multiprocess Nonequilibrium." Water Resources Research 25(9):1971-1988.

Brusseau ML, RE Jessup, and PS Rao. 1993. “Modeling Solute Transport Influenced by Multiprocess Nonequilibrium and Transformation Reactions." Water Resources Research 29(7):2469-2469.

Buckingham E. 1907. "The Capillary Potential." Chapter II in Studies on the Movement of Soil Moisture, pp. 28-61. Bulletin 38, Bureau of Soils, Department of Agriculture, Washington, D.C. Available from http://trid.trb.org/view/124797 (April 2013).

Bunn A, D Wellman, R Deeb, E Hawley, M Truex, M Peterson, M Freshley, E Pierce, J McCord, M Young, T Gilmore, R Miller, A Miracle, D Kaback, C Eddy-Dilek, J Rossabi, M Lee, R Bush, P Beam, G Chamberlain, J Marble, L Whitehurst, K Gerdes, and Y Collazo. 2012. Scientific Opportunities for Monitoring at Environmental Remediation Sites (SOMERS): Integrated Systems-Based Approaches to Monitoring. PNNL-21379, Pacific Northwest National Laboratory, Richland, Washington.

Cohen RM and JW Mercer. 1990. "Review of Immiscible Fluids in the Subsurface: Properties, Models, Characterization and Remediation." Journal of Contaminant Hydrology 6(2):107-163.

Darcy H. 1856. De' termination des lois d'e' coulement de l'eau a' travers le sable. Les fountaines publiques de la ville de Dijon. Victor Dalmont, Paris.

DOE - U.S. Department of Energy. 2010. Long-Range Deep Vadose Zone Program Plan. DOE/RL-2010-89, Richland Operations Office, Richland, Washington. 
DOE - U.S. Department of Energy. 2012. Regulatory Basis and Implementation of a Graded Approach to Evaluation of Groundwater Protection. DOE/RL-2011-50, Revision 1, Richland Operations Office, Richland, Washington.

EPA - U.S. Environmental Protection Agency. 1999. Use of Monitored Natural Attenuation at Superfund, RCRA Corrective Action, and Underground Storage Tank Sites. OSWER Directive 9200.4-17P, Office of Solid Waste and Emergency Response, U.S. Environmental Protection Agency, Washington, D.C.

EPA - U.S. Environmental Protection Agency. 2007a. Monitored Natural Attenuation of Inorganic Contaminants in Ground Water - Volume 1, Technical Basis for Assessment. EPA/600/R-07/139, U.S. Environmental Protection Agency, Washington, D.C.

EPA - U.S. Environmental Protection Agency. 2007b. Monitored Natural Attenuation of Inorganic Contaminants in Ground Water - Volume 2, Assessment for Non-Radionuclides Including Arsenic, Cadmium, Chromium, Copper, Lead, Nickel, Nitrate, Perchlorate, and Selenium. EPA/600/R-07/140, U.S. Environmental Protection Agency, Washington, D.C.

EPA - U.S. Environmental Protection Agency. 2010. Monitored Natural Attenuation of Inorganic Contaminants in Ground Water - Volume 3, Assessment for Radionuclides Including Tritium, Radon, Strontium, Technetium, Uranium, Iodine, Radium, Thorium, Cesium, and Plutonium-Americium. EPA/600/R-10/093, U.S. Environmental Protection Agency, Washington, D.C.

Eslinger PW, CT Kincaid, WE Nichols, and SK Wurstner. 2006. A Demonstration of the System Assessment Capability (SAC) Rev. 1 Software for the Hanford Remediation Assessment Project. PNNL-16209, Pacific Northwest National Laboratory, Richland, Washington.

Fayer MJ, AL Ward, and VL Freedman. 2010. Technical Basis for Evaluating Surface Barriers to Protect Groundwater from Deep Vadose Zone Contamination. PNNL-18661 Final, Pacific Northwest National Laboratory, Richland, Washington.

Freeze RA and JA Cherry. 1979. Groundwater. Prentice-Hall, Englewood Cliffs, New Jersey.

Gee GW, M Oostrom, MD Freshley, ML Rockhold, and JM Zachara. 2007. "Hanford site vadose zone studies: An overview." Vadose Zone Journal 6(4):899-905.

Glass RJ, GH Oosting, and TS Steenhuis. 1989a. "Preferential Solute Transport in Layered Homogeneous Sands as a Consequence of Wetting Front Instability." Journal of Hydrology 110(1-2):87-105.

Glass RJ, J-Y Parlange, and TS Steenhuis. 1989b. "Wetting Front Instability. 1. Theoretical Discussion and Dimensional Analysis.” Water Resources Research 25(6):1187-1194.

Glass RJ, TS Steenhuis, and J-Y Parlange. 1989c. "Mechanism for Finger Persistence in Homogeneous, Unsaturated, Porous-Media - Theory and Verification.” Soil Science 148(1):60-70. 
Glass RJ, TS Steenhuis, and J-Y Parlange. 1989d. "Wetting Front Instability. 2. Experimental Determination of Relationships between System Parameters and Two-Dimensional Unstable Flow Field Behavior in Initially Dry Porous Media.” Water Resources Research 25(6):1195-1207.

Glass RJ, S Cann, J King, N Baily, J-Y Parlange, and TS Steenhuis. 1990. "Wetting Front Instability in Unsaturated Porous-Media: A Three-Dimensional Study in Initially Dry Sand." Transport in Porous Media 5(3):247-268.

Glass RJ, J-Y Parlange, and TS Steenhuis. 1991. "Immiscible Displacement in Porous Media: Stability Analysis of Three-Dimensional, Axisymmetrical Disturbances with Application to Gravity-Driven Wetting Front Instability." Water Resources Research 27(8):1947-1956.

Hillel D. 1998. Environmental Soil Physics: Fundamentals, Applications, and Environmental Considerations. Academic Press, San Diego, California.

Hu QH and JSY Wang. 2003. "Aqueous-Phase Diffusion in Unsaturated Geologic Media: A Review." Critical Reviews in Environmental Science and Technology 33(3):275-297.

ITRC - The Interstate Technology \& Regulatory Council. 2010. A Decision Framework for Applying Monitored Natural Attenuation Processes to Metals and Radionuclides in Groundwater. APMR-1, Attenuation Processes for Metals and Radionuclides Team, Interstate Technology \& Regulatory Council, Washington, D.C. Accessed April 8, 2013, at http://www.itrcweb.org/Guidance/ListDocuments?TopicID=30\&SubTopicID=20.

Jansik D, J Istok, D Wellman, and E Cordova. "The impact of water content on the transport of technetium in Hanford sediments." Vadose Zone Journal in review.

Kechavarzi C, K Soga, and TH Illangasekare. 2005. "Two-dimensional laboratory simulation of LNAPL infiltration and redistribution in the vadose zone." Journal of Contaminant Hydrology 76(3-4):211-233.

Lenhard RJ. 1992. "Measurement and modeling of three-phase saturation-pressure hysteresis." Journal of Contaminant Hydrology 9(3):243-269.

Lenhard RJ, M Oostrom, and JH Dane. 2004. "A constitutive model for air-NAPL-water flow in the vadose zone accounting for immobile, non-occluded (residual) NAPL in strongly water-wet porous media." Journal of Contaminant Hydrology 71(1-4):261-282.

Lindenmeier CW, RJ Serne, BN Bjornstad, GW Gee, HT Schaef, DC Lanigan, MJ Lindberg, RE Clayton, VL Legore, IV Kutnyakov, SR Baum, KN Geiszler, CF Brown, MM Valenta, TS Vickerman, and LJ Royack. 2008. Characterization of Vadose Zone Sediment: RCRA Borehole 299-E33-338 Located Near the B-BX-BY Waste Management Area. PNNL-14121, Rev. 1, Pacific Northwest National Laboratory, Richland, Washington.

Mayer A, T Sandman, and M Breidenbach. 2008. "Effect of flow regime on physical nonequilibrium transport in unsaturated porous media." Vadose Zone Journal 7(3):981-991. 
Murray CJ, AL Ward, and JL Wilson, III. 2003. Influence of Clastic Dikes on Vertical Migration of Contaminants in the Vadose Zone at Hanford. PNNL-14224, Pacific Northwest National Laboratory, Richland, Washington.

Oostrom M, PD Thorne, ZF Zhang, GV Last, and MJ Truex. 2008. Modeling of Carbon Tetrachloride Flow and Transport in the Subsurface of the 200 West Disposal Sites: Large-Scale Model Configuration and Prediction of Future Carbon Tetrachloride Distribution Beneath the 216-Z-9 Disposal Site. PNNL-17181, Pacific Northwest National Laboratory, Richland, Washington.

Padilla IY, TCJ Yeh, and MH Conklin. 1999. "The effect of water content on solute transport in unsaturated porous media." Water Resources Research 35(11):3303-3313.

Parlange J-Y, RJ Glass, and TS Steenhuis. 1990. "Application of scaling to the analysis of unstable flow phenomena." Chapter 5 in Scaling in Soil Physics: Principles and Applications, D Hillel and DE Elrick (eds), pp. 53-57. Special Publication 25, Soil Science Society of America, Madison, Wisconsin.

Rasmuson A and JC Eriksson. 1988. "On the Physicochemical Basis for the Capillary Barrier Effect." Nordic Hydrology 19(5):281-292.

Serne RJ, GV Last, GW Gee, HT Schaef, DC Lanigan, CW Lindenmeier, MJ Lindberg, RE Clayton, VL Legore, RD Orr, IV Kutnyakov, SR Baum, KN Geiszler, CF Brown, MM Valenta, and TS Vickerman. 2008. Characterization of Vadose Zone Sediment: Borehole 299-E33-45 Near BX-102 in the $B-B X-B Y$ Waste Management Area. PNNL-14083, Rev. 1, Pacific Northwest National Laboratory, Richland, Washington.

Serne RJ, AL Ward, W Um, BN Bjornstad, DF Rucker, DC Lanigan, and MW Benecke. 2009. Electrical Resistivity Correlation to Vadose Zone Sediment and Pore-Water Composition for the BC Cribs and Trenches Area. PNNL-17821, Pacific Northwest National Laboratory, Richland, Washington.

Serne R, B Bjornstad, J Keller, P Thorne, D Lanigan, J Christensen, and G Thomas. 2010. Conceptual Models for Migration of Key Groundwater Contaminants Through the Vadose Zone and Into the Upper Unconfined Aquifer Below the B-Complex. PNNL-19277, Pacific Northwest National Laboratory, Richland, Washington.

Soil Science Society of America. 1981. Water Potential Relations in Soil Microbiology. Special Publication \#9, Soil Science Society of America, Madison, Wisconsin.

Thorne PD, MP Bergeron, MD Williams, and VL Freedman. 2006. Groundwater Data Package for Hanford Assessments. PNNL-14753, Rev. 1, Pacific Northwest National Laboratory, Richland, Washington.

Toride N, M Inoue, and FJ Leij. 2003. "Hydrodynamic dispersion in an unsaturated dune sand." Soil Science Society of America Journal 67(3):703-712. 
Truex M, P Brady, C Newell, M Rysz, M Denham, and K Vangelas. 2011a. The Scenarios Approach to Attenuation Based Remedies for Inorganic and Radionuclide Contaminants. SRNL-STI-2011-00459, Savannah River National Laboratory, Aiken, South Carolina. Available at http://sti.srs.gov/fulltext/ SRNL-STI-2011-00459.pdf. doi: 10.2172/1023615.

Truex MJ, M Oostrom, VL Freedman, CE Strickland, TW Wietsma, GD Tartakovsky, and AL Ward. 2011b. Laboratory and Modeling Evaluations in Support of Field Testing for Desiccation at the Hanford Site. PNNL-20146, Pacific Northwest National Laboratory, Richland, Washington.

Truex MJ, M Oostrom, CE Strickland, TC Johnson, VL Freedman, CD Johnson, WJ Greenwood, AL Ward, RE Clayton, MJ Lindberg, JE Peterson, SS Hubbard, GB Chronister, and MW Benecke. 2012. Deep Vadose Zone Treatability Test for the Hanford Central Plateau: Soil Desiccation Pilot Test Results. PNNL-21369, Pacific Northwest National Laboratory, Richland, Washington.

Ward AL, GW Gee, ZF Zhang, and JM Keller. 2004. Vadose Zone Contaminant Fate and Transport Analysis for the 216-B-26 Trench. PNNL-14907, Pacific Northwest National Laboratory, Richland, Washington.

WAC 173-340. 2007. "Model Toxics Control Act - Cleanup." Washington Administrative Code, Olympia, Washington. 
PNNL-21815

RPT-DVZ-AFRI-004

\section{Distribution}

No. of

Copies

ONSITE

3 U.S. Department of Energy, Richland Operations Office

DOE Public Reading Room

BL Charboneau

JG Morse
No. of

Copies

3 Pacific Northwest National Laboratory

MJ Truex (2)

K6-96

DM Wellman

K3-62

$\mathrm{H} 2-53$

A6-33

A5-11

Distr.1 


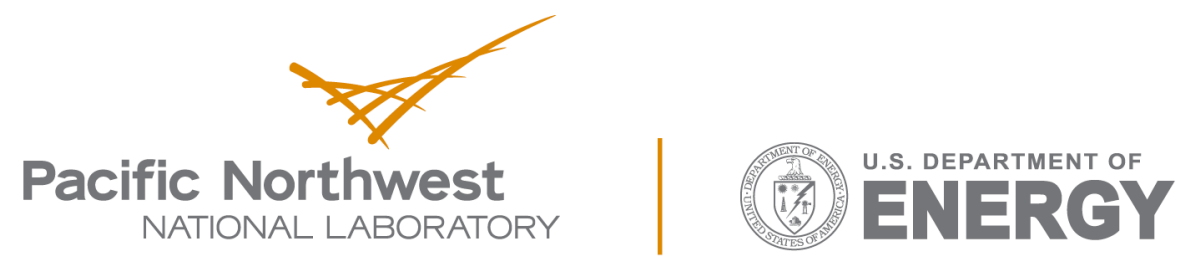

Proudly Operated by Battelle Since 1965

902 Battelle Boulevard

P.O. Box 999

Richland, WA 99352

1-888-375-PNNL (7665)

www.pnnl.gov 